\title{
CHEMICAL CONSTITUENTS IN WATER FROM WELLS IN THE VICINITY OF THE NAVAL REACTORS FACILITY, IDAHO NATIONAL ENGINEERING LABORATORY, IDAHO, 1991-93
}

By Betty J. Tucker, LeRoy L. Knobel, and Roy C. Bartholomay

\section{U.S. GEOLOGICAL SURVEY}

Open-File Report 95-725

Prepared in cooperation with the U.S. DEPARTMENT OF ENERGY

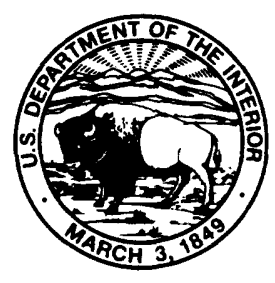




\title{
U.S. DEPARTMENT OF THE INTERIOR \\ BRUCE BABBITT, Secretary
}

\author{
U.S. GEOLOGICAL SURVEY \\ GORDON P. EATON, Director
}

Any use of trade, product, or firm names in this publication is for descriptive purposes only and does not constitute endorsement by the U.S. Government.

For additional information write to:

U.S. Geological Survey

INEL, MS 4148

P.O. Box 2230

Idaho Falls, ID 83403-2230
Copies of this report can be purchased from:

U.S. Geological Survey

Earth Science Information Center

Open-File Reports Section

Box 25286, MS 517, Denver Federal Center

Denver, CO 80225 


\section{CONTENTS}

Abstract

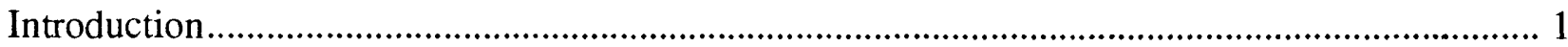

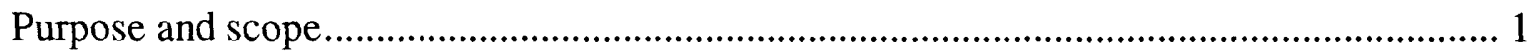

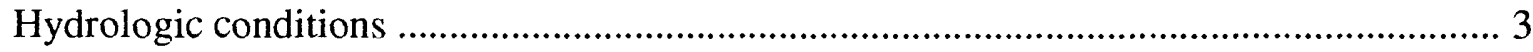

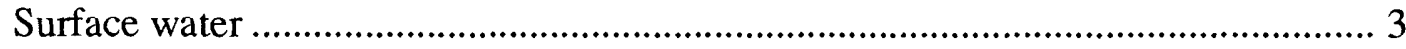

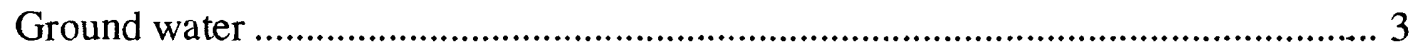

Guidelines for interpreting results of radiochemical analyses........................................ 4

Guidelines for interpreting results of inorganic and organic analyses............................... 5

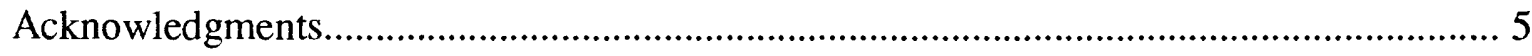

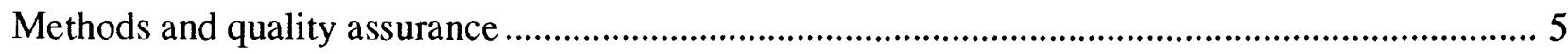

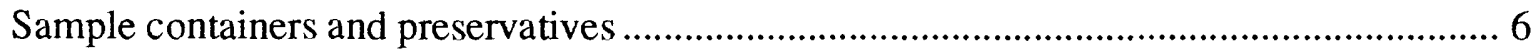

Sampling locations and sample collection ........................................................... 6

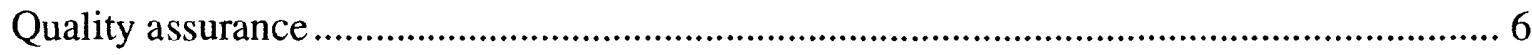

Calculation of estimated experimental standard errors........................................... 8

Analytical results for round-three water samples ........................................................... 9

Dissolved anions and total recoverable sodium....................................................... 9

Total recoverable trace elements ............................................................................. 9

Dissolved nutrients................................................................................................ 10

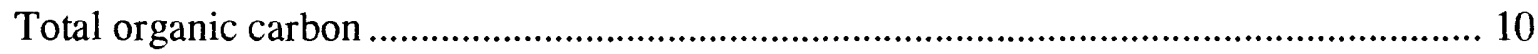

Gross alpha- and gross beta-particle radioactivity .................................................. 10

Analytical results for round-two water samples ............................................................... 12

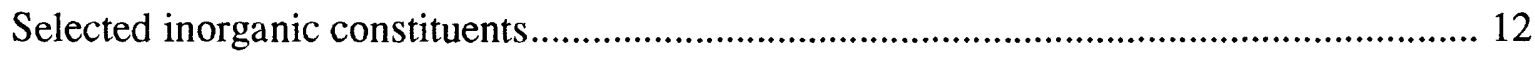

Total organic carbon and phenols .......................................................................... 12

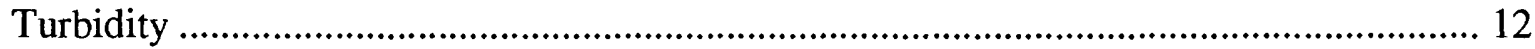

Extractable acid and base/neutral organic compounds .............................................. 12

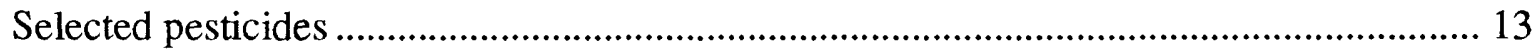

Gross alpha- and gross beta-particle radioactivity ................................................. 13

Selected dissolved radium isotopes ...................................................................... 13

Analytical results for round-one water samples................................................................ 13

Selected inorganic constituents.......................................................................... 14

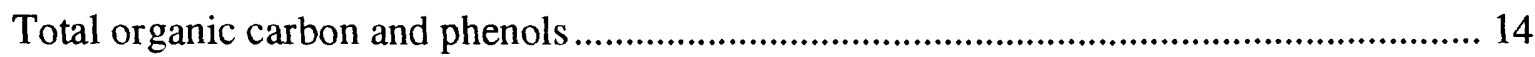

Total recoverable anionic surfactants and turbidity ................................................ 14

Extractable acid and base/neutral organic compounds ............................................... 14

Purgeable organic compounds .............................................................................. 14

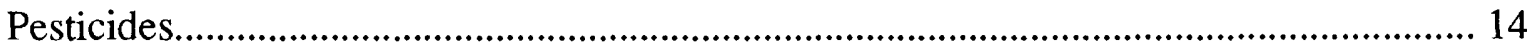

Gross alpha- and gross beta-particle radioactivity .............................................. 15

Selected dissolved radium isotopes and tritium.......................................................... 15

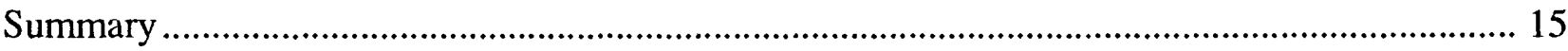

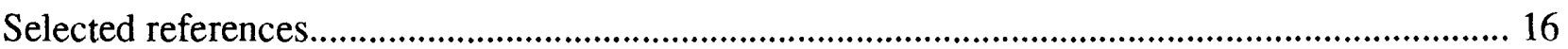




\section{ILLUSTRATIONS}

Figures 1-2. Maps showing:

1. Location of the Idaho National Engineering Laboratory, Naval Reactors

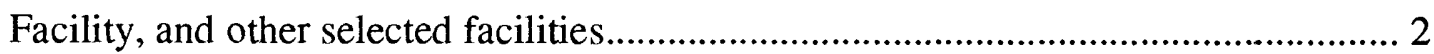

2. Location of wells, Naval Reactors Facility and vicinity, Idaho National

Engineering Laboratory 7

\section{TABLES}

Table 1. Containers and preservatives used for water samples, Naval Reactors Facility and vicinity.....

2. Results of field measurements for $\mathrm{pH}$, specific conductance, and temperature of water, Naval Reactors Facility and vicinity

3. Concentrations of dissolved anions and total recoverable sodium in water from round-three samples, Naval Reactors Facility and vicinity

4. Statistical parameters for dissolved anions and total recoverable sodium, by well......... 35

5. Concentrations of selected total recoverable trace elements in water from round-three samples, Naval Reactors Facility and vicinity 39

6. Statistical parameters for selected total recoverable trace elements, by well

7. Concentrations of dissolved nutrients and total organic carbon in water from round-three samples, Naval Reactors Facility and vicinity.....

8. Statistical parameters for dissolved nutrients, total organic carbon, total phenols, and turbidity, by well

9. Concentrations of gross alpha-particle radioactivity in water from round-three samples, Naval Reactors Facility and vicinity

10. Concentrations of gross beta-particle radioactivity in water from round-three samples, Naval Reactors Facility and vicinity

11. Statistical parameters for gross alpha-particle radioactivity, gross beta-particle radioactivity, radium-226, and radium-228, by well

12. Concentrations of selected inorganic constituents in water from round-two samples, wells NRF-6 and NRF-7

13. Concentrations of total organic carbon and total phenols in water, and turbidity, from round-two samples, wells NRF-6 and NRF-7.

14. Extractable acid and base/neutral organic compounds for which round-one and round-two water samples were analyzed...

15. Concentrations of extractable acid and base/neutral organic compounds in water from round-one and round-two samples, wells NRF-6 and NRF-7.

16. Pesticides for which round-two water samples were analyzed 84

17. Concentrations of radioactivity, dissolved radium-226, and dissolved radium- 228 in water from round-two samples, wells NRF-6 and NRF-7

18. Concentrations of selected inorganic constituents in water from round-one samples, wells NRF-6 and NRF-7 
19. Concentrations of total recoverable anionic surfactants, total organic carbon, and total phenols in water, and turbidity of water, from round-one samples, wells NRF-6 and NRF-7

20. Purgeable organic compounds for which round-one water samples were analyzed....... 90

21. Herbicides for which round-one water samples were analyzed ...................................... 91

22. Insecticides, benzene hexachlorides, gross polychlorinated compounds, and aroclors for which round-one water samples were analyzed

23. Concentrations of radioactivity and selected radionuclides in water from round-one samples, wells NRF-6 and NRF-7

\section{CONVERSION FACTORS, VERTICAL DATUM, AND ABBREVIATED UNITS}

Multiply

foot $(\mathrm{ft})$

inch (in.)

mile (mi)

square mile $\left(\mathrm{mi}^{2}\right)$

acre-foot (acre-ft)

foot per mile (ft/mi)

picocurie per liter $(\mathrm{pCi} / \mathrm{L})$
$\underline{B y}$

0.3048

25.4

1.609

2.590

1,233

0.1894

0.037
To obtain

meter

millimeter

kilometer

square kilometer

cubic meter

meter per kilometer

becquerel per liter

For temperature, degrees Celsius $\left({ }^{\circ} \mathrm{C}\right)$ can be converted to degrees Fahrenheit $\left({ }^{\circ} \mathrm{F}\right)$ by using the equation: ${ }^{\circ} \mathrm{F}=(1.8)\left({ }^{\circ} \mathrm{C}\right)+32$.

Sea level: In this report, "sea level" refers to the National Geodetic Vertical Datum of 1929--a geodetic datum derived from a general adjustment of the first-order level nets of the United States and Canada, formerly called Sea Level Datum of 1929.

Abbreviated units used in report: $\mathrm{mg} / \mathrm{L}$ (milligram per liter); $\mu \mathrm{g} / \mathrm{L}$ (microgram per liter); and $\mu \mathrm{S} / \mathrm{cm}$ (microsiemens per centimeter at 25 degrees Celsius). 



\title{
CHEMICAL CONSTITUENTS IN WATER FROM WELLS IN THE VICINITY OF THE NAVAL REACTORS FACILITY, IDAHO NATIONAL ENGINEERING LABORATORY, IDAHO, 1991-93
}

\author{
by Betty J. Tucker, LeRoy L. Knobel, and Roy C. Bartholomay
}

\section{Abstract}

The U.S. Geological Survey, in response to a request from the U.S. Department of Energy's Pittsburgh Naval Reactors Office, Idaho Branch Office, sampled 14 wells during 1991-93 as part of a long-term project to monitor water quality of the Snake River Plain aquifer in the vicinity of the Naval Reactors Facility, Idaho National Engineering Laboratory, Idaho. Water samples were analyzed for manmade contaminants and naturally occurring constituents. One hundred sixty-one samples were collected from 10 ground-water monitoring wells and 4 production wells. Twenty-one quality-assurance samples also were collected and analyzed; 2 were blank samples and 19 were replicate samples. The two blank samples contained concentrations of six inorganic constituents that were slightly greater than the laboratory reporting levels (the smallest measured concentration of a constituent that can be reported using a given analytical method). Concentrations of other constituents in the blank samples were less than their respective reporting levels. The 19 replicate samples and their respective primary samples generated 614 pairs of analytical results for a variety of chemical and radiochemical constituents. Of the 614 data pairs, 588 were statistically equivalent at the 95 -percent confidence level; about 96 percent of the analytical results were in agreement. Two pairs of turbidity measurements were not evaluated because of insufficient information and one primary sample collected in January 1992 contained tentatively identified organic compounds when the replicate sample did not.

\section{INTRODUCTION}

The Idaho National Engineering Laboratory (INEL), encompassing about $890 \mathrm{mi}^{2}$ of the eastern Snake River Plain in southeastern Idaho (fig. 1), is operated by the U.S. Department of Energy (DOE). INEL facilities are used in the development of peacetime atomicenergy applications, nuclear safety research, defense programs, and advanced energy concepts. Activities at the Naval Reactors Facility (NRF), one facility at the INEL, have involved previous limited releases of some constituents to the environment as described in the NRF history report (Bettis Atomic Power Laboratory, 1992).

This study was conducted by the U.S. Geological Survey (USGS) in cooperation with the DOE's Pittsburgh Naval Reactors Office, Idaho Branch Office (IBO). IBO is responsible for the Naval Reactors Facility at the INEL. That office requires information about the mobility of radionuclide- and chemical-waste constituents in the Snake River Plain aquifer. Waste-constituent mobility is, in part, determined by (1) the rate and direction of ground-water flow; (2) the locations, quantities, and methods of waste disposal; (3) waste-constituent chemistry; and (4) the geochemical processes taking place in the aquifer (Orr and Cecil, 1991, p. 2).

\section{Purpose and Scope}

In 1989, the IBO of the Pittsburgh Naval Reactors Office, DOE, requested that the USGS initiate a water-quality data-collection program in the vicinity of the Naval Reactors 


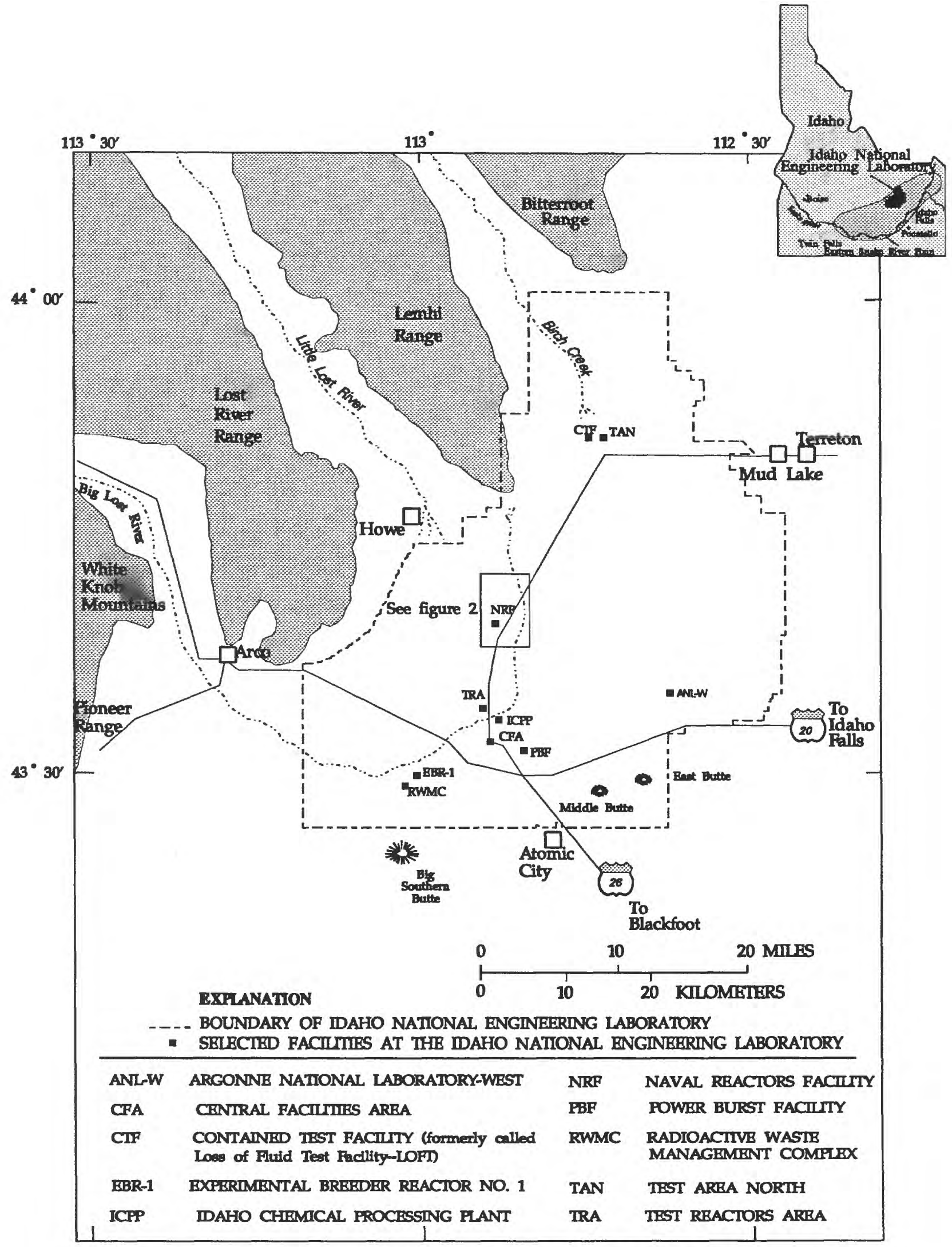

Figure 1.-Location of the Idaho National Engineering Laboratory, Naval Reactors Facility, and other selected facilities. 
Facility at the INEL (fig. 1). The purpose of the data-collection program is to provide the Idaho Branch Office with water-chemistry data to evaluate the impact of NRF activities on the water quality of the Snake River Plain aquifer.

The sample-collection program consists of three rounds of data collection. Round one was a one-time sampling of each well for a comprehensive suite of chemical constituents that approximates those contained in the U.S. Environmental Protection Agency's GroundWater Monitoring List—Appendix IX (U.S. Environmental Protection Agency, 1989, p. 636-642). Round two consisted of five bimonthly samples from each well that were analyzed for the chemical constituents listed in Appendix III-EPA Interim Primary Drinking Water Standards, the constituents listed as parameters establishing ground-water quality, and selected measurements used as indicators of ground-water contamination (U.S. Environmental Protection Agency, 1989, p. 660-661, 730). Additional constituents analyzed for in round-two samples included copper, nickel, zinc, and extractable acid and base/neutral compounds. Round three is ongoing; samples are collected on a quarterly basis. Constituents include chloride, chromium, iron, lead, mercury, nickel, nitrate as nitrogen, silver, sodium, and sulfate. Other round-three measurements are gross alpha- and gross beta-particle radioactivity, $\mathrm{pH}$, specific conductance, and total organic carbon (TOC). As a result of expanded laboratory procedures, all three rounds of the sample-collection program include analyses for constituents in addition to those described above.

This report presents a compilation of roundone, round-two, and round-three waterchemistry data collected during 1991-93. Additional round-three water-chemistry data will be presented in subsequent reports.

\section{Hydrologic Conditions}

The Snake River Plain aquifer is one of the most productive aquifers in the United States (U.S. Geological Survey, 1985, p. 193). The aquifer underlies the eastern Snake River Plain and consists of a thick sequence of basalts and sedimentary interbeds filling a large, arcuate, structural basin in southeastern Idaho (fig. 1).

\section{Surface Water}

The Big Lost River drains more than 1,400 $\mathrm{mi}^{2}$ of mountainous area that includes parts of the Lost River Range and the Pioneer Range west of the INEL (fig. 1). Flow in the Big Lost River infiltrates to the Snake River Plain aquifer along its channel and at sinks and playas at the river's terminus. Since 1958, excess runoff has been diverted to spreading areas in the southwestern part of the INEL where much of the water rapidly infiltrates to the aquifer. Other surface drainages that provide recharge to the Snake River Plain aquifer at the INEL include Birch Creek and the Little Lost River (fig. 1) (Orr and Cecil, 1991, p. 23).

\section{Ground Water}

Recharge to the Snake River Plain aquifer is principally from infiltration of applied irrigation water, infiltration of streamflow, and alluvial ground-water inflow from adjoining mountain drainage basins. Some recharge may be from direct infiltration of precipitation, although the small amount of annual precipitation on the plain ( 8 in. at the INEL), evapotranspiration, and the great depth to water (in places exceeding $900 \mathrm{ft}$ ) probably minimize this source of recharge (Orr and Cecil, 1991, p. 22-23).

Water in the Snake River Plain aquifer moves principally through fractures and interflow zones in the basalt. A significant proportion of ground water moves through the upper $800 \mathrm{ft}$ of saturated rocks. Hydraulic conductiv- 
ities of basalt in the upper $800 \mathrm{ft}$ of the aquifer, estimated from INEL-wide transmissivity data, range from 0.0086 to $5,500 \mathrm{ft} /$ day (Ackerman, 1991, p. 30). Hydraulic conductivities estimated in a 10,365 -ft deep test hole near NRF are smaller; at depths exceeding $1,500 \mathrm{ft}$, hydraulic conductivities range from 0.002 to $0.03 \mathrm{ft} /$ day. The effective base of the Snake River Plain aquifer at the INEL probably ranges from about 850 to $1,220 \mathrm{ft}$ below land surface (Mann, 1986, p. 21).

Depth to water in wells completed in the Snake River Plain aquifer ranges from about $200 \mathrm{ft}$ in the northern part of the INEL to more than $900 \mathrm{ft}$ in the southeastern part; in the vicinity of NRF, depth to water is about $375 \mathrm{ft}$. In July 1988, the altitude of the water table was about $4,590 \mathrm{ft}$ above sea level near Test Area North (TAN) and about 4,420 ft above sea level near the Radioactive Waste Management Complex (RWMC); near the NRF, the altitude was about $4,500 \mathrm{ft}$ above sea level. Water flowed southward and southwestward beneath the INEL at an average hydraulic gradient of about $4 \mathrm{ft} / \mathrm{mi}$; beneath the NRF, water generally flowed southward. Locally, however, the hydraulic gradient ranged from about 1 to $15 \mathrm{ft} / \mathrm{mi}$. From July 1985 to July 1988, water-level changes in INEL wells ranged from a 26.8 -ft decline near the RWMC to a 4.3-ft rise north of TAN; near the NRF, the water-level decline was about 1 to $3 \mathrm{ft}$. Water levels generally declined in the southern twothirds of the INEL during that time and rose in the northern one-third (Orr and Cecil, 1991, p. 25-27).

Ground water moves southwestward from the INEL and eventually discharges to springs along the Snake River downstream from Twin Falls, about $100 \mathrm{mi}$ southwest of the INEL. Approximately 4.3 million acre-ft of ground water discharged to these springs in 1988 (Mann, 1989, p. 2).

\section{Guidelines for Interpreting Results of Radiochemical Analyses}

Concentrations of radionuclides are reported with an estimated sample standard deviation, $\mathbf{s}$, that is obtained by propagating sources of analytical uncertainty in measurements. The following guidelines for interpreting analytical results are based on an extension of a method proposed by Currie (1984).

In the analysis for a particular radionuclide, laboratory measurements are made on a target sample and a prepared blank. Instrument signals for the sample and the blank vary randomly. Therefore, it is essential to distinguish between two key aspects of the problem of detection: (1) the instrument signal for the sample must be larger than the signal observed for the blank before the decision can be made that the radionuclide was detected; and (2) an estimation must be made of the minimum radionuclide concentration that will yield a sufficiently large observed signal before the correct decision can be made for detection or nondetection of the radionuclide. The first aspect of the problem is a qualitative decision based on an observed signal and a definite criterion for detection. The second aspect of the problem is an estimation of the detection capabilities of a given measurement process.

In the laboratory, instrument signals must exceed a critical level of $1.6 \mathrm{~s}$ before the qualitative decision can be made as to whether the radionuclide was detected. At $1.6 \mathrm{~s}$, there is a 95-percent probability that the correct conclusion-not detected-will be made. Given a large number of samples, as many as 5 percent of the samples with measured concentrations larger than or equal to $1.6 \mathrm{~s}$, which were concluded as being detected, might not contain the radionuclide. These measurements are referred to as false positives and are errors of the first kind in hypothesis testing. 
Once the critical level of $1.6 \mathrm{~s}$ has been defined, the minimum detectable concentration may be determined. Radionuclide concentrations that equal $3 \mathrm{~s}$ represent a measurement at the minimum detectable concentration. For true concentrations of $3 \mathbf{s}$ or larger, there is a 95-percent or larger probabiliiy that the radionuclide was detected in a sample. In a large number of samples, the conclusion-not detected-will be made in 5 percent of the samples that contain true concentrations at the minimum detectable concentration of $3 \mathrm{~s}$. These measurements are referred to as false negatives and are errors of the second kind in hypothesis testing.

True radionuclide concentrations between $1.6 \mathrm{~s}$ and $3 \mathrm{~s}$ have larger errors of the second kind. That is, there is a larger-than-5-percent probability of false negative results for samples with true concentrations between $1.6 \mathrm{~s}$ and 3s. Although the radionuclide might have been detected, such detection may not be considered reliable; at $1.6 \mathrm{~s}$, the probability of a false negative is about 50 percent.

The critical level and minimum detectable concentration are based on counting statistics alone and do not include systematic or random errors inherent in laboratory procedures. The values $1.6 \mathrm{~s}$ and $3 \mathrm{~s}$ vary slightly with background or blank counts, with the number of gross counts for individual analyses, and for different radionuclides. In this report, radionuclide concentrations less than $3 \mathrm{~s}$ are considered to be below a "reporting level." The critical level, minimum detectable concentration, and reporting level aid the reader in the interpretation of analytical results and do not represent absolute concentrations of radioactivity which may or may not have been detected.

Many analytical results of environmental radioactivity measurements are at or near zero. If the true concentration for a given radionuclide is zero, a given set of analytical results for that radionuclide should be distributed about zero, with an equal number of negative and positive measurements. Negative analytical results occur if the radioactivity of a water sample is less than the background radioactivity or the radioactivity of the prepared blank sample in the laboratory (American Society for Testing and Materials, 1992, p. 126; Knobel and others, 1992, p. 50).

\section{Guidelines for Interpreting Results of Inorganic and Organic Analyses}

The term "reporting level" used for radiochemical analyses should not be confused with the term "laboratory reporting level", which is used for inorganic and organic analyses. In this report the term "laboratory reporting level" is the smallest measured concentration of a nonradioactive constituent that may be reliably reported using a given analytical method. Because of unpredictable matrix effects on detection limits, the laboratory reporting levels are set somewhat higher than the analytical method detection limits (Pritt and Jones, 1989).

\section{Acknowledgments}

Steven J. Price, Kent R. Shelley, and Kelly D. Willie of Westinghouse Electric Corporation provided logistical support during sample collection. The authors are grateful to Linda M. Williams of the USGS and Bruce E. Olenick, Adolfo Sierra, and Kelly D. Willie of Westinghouse Electric Corporation for technically reviewing the manuscript.

\section{METHODS AND QUALITY ASSURANCE}

The methods used for collecting water samples generally followed the guidelines established by the USGS (Goerlitz and Brown, 1972; Stevens and others, 1975; Wood, 1981; Claassen, 1982; W.L. Bradford, USGS, 
written commun., 1985; Wershaw and others, 1987; Fishman and Friedman, 1989; Hardy and others, 1989; Faires, 1992; Fishman, 1993). The methods used in the field and the quality assurance practices are described in following sections.

\section{Sample Containers and Preservatives}

Sample containers and preservatives differed depending on the constituent(s) for which analyses were requested. Samples analyzed by the U.S. Geological Survey's NWQL were placed in containers and preserved in accordance with laboratory requirements specified by Pritt and Jones (1989). Containers and preservatives were supplied by the NWQL and had undergone a rigorous quality-control procedure (Pritt, 1989, p. 75) to eliminate sample contamination. The containers and preservatives used for this study are listed in table 1 (all tables located at the end of this report).

\section{Sampling Locations and Sample Collection}

Samples were collected from 14 locations (fig. 2): 10 ground-water monitoring wells (NRF-6, -7, USGS 12, 15, 17, 97-99, 102, and Water Supply INEL-1), and 4 production wells (NRF-1, -2, -3, and -4). The ground-water monitoring wells were equipped with dedicated submersible pumps and the production wells were equipped with line-shaft turbine pumps. The production wells are located within the NRF boundary; USGS 102 is located west of the boundary; NRF-6, -7 , USGS 12,15 , and 17 are upgradient of the facility; and the remaining monitoring wells are located downgradient (fig. 2).

Samples were collected from a portable sampling apparatus at the wells with dedicated submersible pumps and from sampling ports on the discharge lines of the turbine pumps. All portable equipment was decontaminated after sampling at each site. After collection, sample containers were sealed with laboratory film, labeled, and stored under secured conditions. Water samples were placed in ice chests, sealed, and shipped as soon as possible by overnight-delivery mail to the NWQL.

Conditions at the sampling site during sample collection were recorded in a field logbook and a chain-of-custody record was used to track samples from the time of collection until delivery to the NWQL. These records are available for inspection at the U.S. Geological Survey's Project Office at the INEL. The results of field measurements for $\mathrm{pH}$, specific conductance, and water temperature are listed in table 2.

\section{Quality Assurance}

Detailed descriptions of internal quality control and the overall quality-assurance practices used by the NWQL are provided in reports by Friedman and Erdmann (1982), Jones (1987), and Pritt and Raese (1992). The water samples were collected by personnel assigned to the INEL Project Office in accordance with a draft quality-assurance plan for quality-of-water activities; the draft plan was finalized in June 1989 and is available for inspection at the U.S. Geological Survey's Project Office at the INEL. A comparative study to determine agreement between analytical results for water-sample pairs by laboratories involved in the INEL Project Office's quality-assurance program was summarized by Wegner (1989). Additional quality assurance instituted for this sampling program included a blank sample prepared with organic-free water, an equipment blank prepared with inorganic-free water flushed through the sampling apparatus, and 19 replicate samples. Concentrations in the blank samples were not included in the computation of statistical parameters. Subsequent to collection of the primary (routine) sample, another sample was 


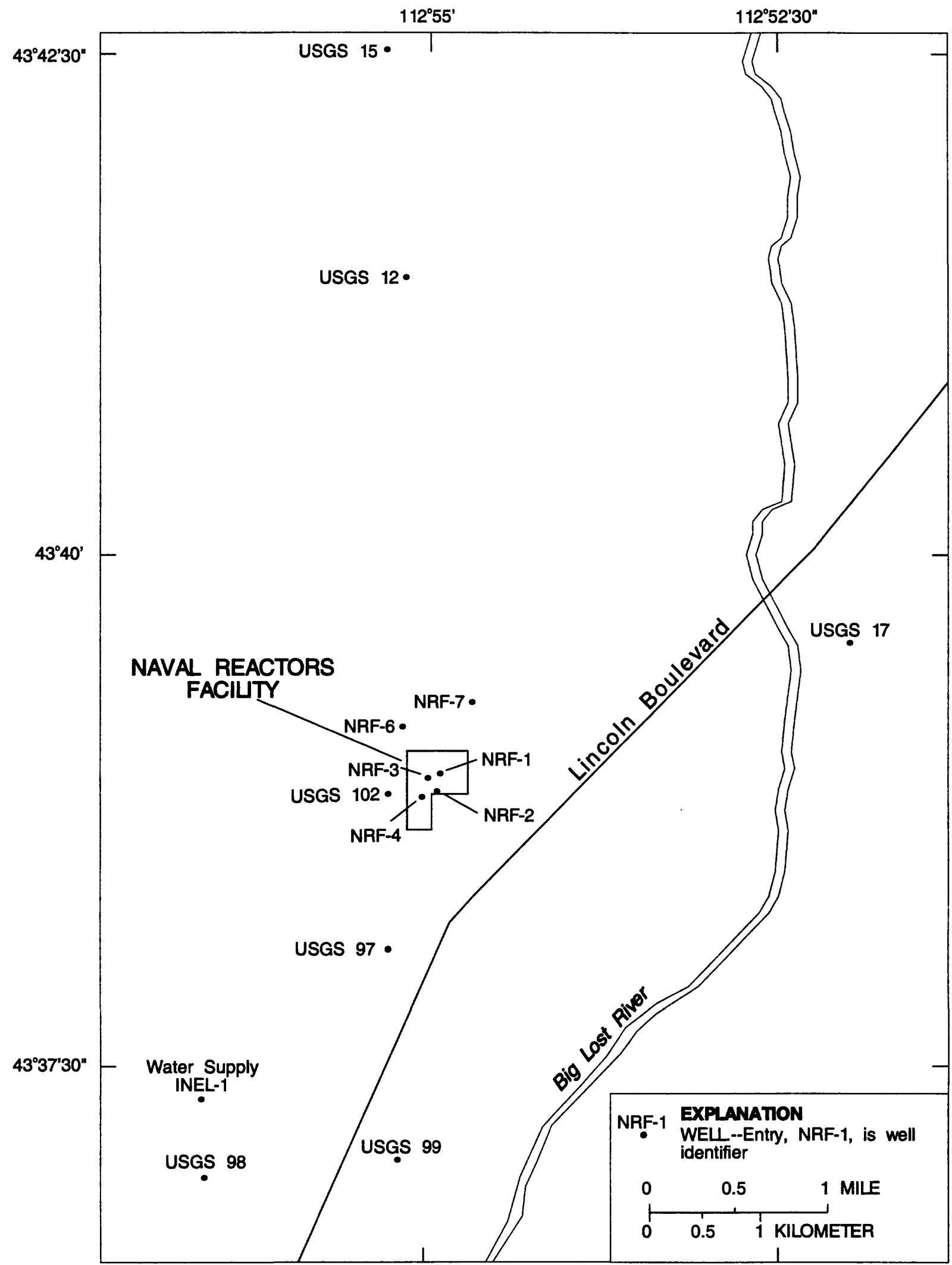

Figure 2. Location of wells, Naval Reactors Facility and vicinity, Idaho National Engineering Laboratory. 
immediately collected and was called the replicate (quality-assurance) sample. The replicate-sample analytical results were then compared to the primary-sample analytical results to evaluate the combined effects of laboratory reproducibility in analytical measurements and of consistency in fieldcollection methods. Many organizations use the term "sequential replicate" in place of "replicate" sample. Analytical results for primary- and replicate-water samples were statistically compared in a study of the qualityassurance and quality-control data collected by the USGS at the INEL during 1989-93 (L.M. Williams, U.S. Geological Survey, written commun., October 11, 1994).

If standard deviations of primary-and replicate-sample results are known, it is possible to determine-within specified confidence limits-whether the results of the pair of samples are statistically equivalent. This can be done using an adaptation of the equation to determine the standard deviate or the number of standard deviations that the variable deviates from the mean (Volk, 1969, p. 55), where $\mathrm{Z}$ is the ratio of the absolute value of the difference of the two results and the pooled standard deviation (Taylor, 1987, p. 29). In that way, a comparison can be made of two analytical results on the basis of the precision-or an approximation of the precision-associated with each of the results:

$$
z=\frac{|x-y|}{\sqrt{\left(s_{x}\right)^{2}+\left(s_{y}\right)^{2}}},
$$

where

$$
\begin{aligned}
& x=\text { result of the primary (routine) sample, } \\
& y=\text { result of the replicate (quality assurance) } \\
& \text { sample, } \\
& S_{x}=\text { standard deviation of } x \text {, and } \\
& S_{y}=\text { standard deviation of } y \text {. }
\end{aligned}
$$

If the $\mathrm{Z}$ value is less than or equal to 1.96 , the analytical results of the primary and replicate pair are considered statistically equivalent at the 95-percent confidence limit.

Equation 1 cannot be applied directly to the results for which no standard deviations or uncertainties are reported. The NWQL does not report standard deviations with analytical results for nonradiochemical constituents; however, the U.S. Geological Survey's Branch of Quality Assurance conducts a Blind Sample Program (BSP) (Maloney and others, 1993) that allows the calculation of a most probable deviation at any concentration for most constituents. A minimum MPD has been established for a few constituents that are generally present at small concentrations (Maloney and others, 1993, p. 4). Linear regression equations generated from BSP data can be used to determine if the analytical results of the primary-and replicate-samples are statistically equivalent by calculating an MPD for each result and substituting them for the standard deviations in equation 1 (L.M. Williams, U.S. Geological Survey, written commun., October 11, 1994). Analytical results for the quality-assurance samples will be discussed, along with similar data, in subsequent sections of this report.

\section{Calculation of Estimated Experimental Standard Errors}

The analytical results for radionuclides are presented with calculated analytical uncertainties. There is about a 67-percent probability that the true radionuclide concentration is in a range of the reported concentration plus or minus the uncertainty. The uncertainties are expressed as one $\mathbf{s}$ (sample standard deviation).

The associated uncertainties presented with mean concentrations are experimental standard errors and are an estimate of the uncertainty of the mean concentration. The estimated experimental standard errors (EESE) were calculated 
using the following equation (Iman and

Conover, 1983, p. 158):

$\operatorname{EESE}=\sigma /(n)^{0.5}$

where $\sigma=$ population standard deviation, and

$\mathrm{n}=$ sample size.

The population standard deviation, $\sigma$, is customarily estimated by $\mathbf{s}$ (Iman and Conover, 1983, p. 106). The sample standard deviation is the square root of the sample variance (Iman and Conover, 1983, p. 100-101).

\section{ANALYTICAL RESULTS FOR ROUND- THREE WATER SAMPLES}

During the period beginning in March 1991 and ending in December 1993, 12 sets of quarterly water samples were collected for round three of the NRF sampling program (table 2). Seven wells (NRF-1, -2, -4, USGS 17, 97, 99, and Water Supply INEL-1) were sampled all 12 times, 4 wells (NRF-3, USGS 12,15 , and 102) were sampled 11 times, USGS 98 was sampled 10 times, NRF-7 was sampled 6 times, and NRF- 6 was sampled 5 times. A quality-assurance blank sample (QAS-23) and an equipment blank (QAS-30) were collected during round three. Seventeen replicate samples were collected during round three as follows: NRF-1 (QAS-18), NRF-2

(QAS-13 and QAS-31), NRF-3 (QAS-16 and QAS-26), NRF-4 (QAS-28), USGS 12 (QAS-17 and QAS-33), USGS 15 (QAS-22), USGS 17 (QAS-14 and QAS-29), USGS 97 (QAS-15 and QAS-32), USGS 98 (QAS-25), USGS 99 (QAS-24), USGS 102 (QAS-27), and Water Supply INEL-1 (QAS-19).

\section{Dissolved Anions and Total Recoverable Sodium}

Round-three water samples were analyzed for concentrations of dissolved bromide, chloride, fluoride, and sulfate, and concentra- tions of total recoverable sodium (table 3 ). Statistical parameters for these constituents are provided by well in table 4 and were calculated using the data presented in this report.

The concentrations in the equipment blank (QAS-30) were all less than or near to the respective laboratory reporting levels. The blank sample (QAS-23) contained concentrations of bromide $(0.07 \mathrm{mg} / \mathrm{L})$, chloride $(0.8 \mathrm{mg} / \mathrm{L})$, and sodium $(0.7 \mathrm{mg} / \mathrm{L})$ that were larger than the laboratory reporting levels of $0.01,0.1$, and $0.1 \mathrm{mg} / \mathrm{L}$, respectively. Eightytwo of 85 replicate-sample concentrations listed in table 3 were statistically equivalent to their primary-sample concentrations. The bromide concentration of QAS-22 $(0.01 \mathrm{mg} / \mathrm{L})$ was not statistically equivalent to the bromide concentration in the primary water sample, USGS $15(0.02 \mathrm{mg} / \mathrm{L})$. The sodium concentraions of two replicate samples were not statistically equivalent to the sodium concentrations in their primary water samples: QAS-22 $(8.7 \mathrm{mg} / \mathrm{L})$ and USGS $15(5.7 \mathrm{mg} / \mathrm{L})$; and QAS-25 $(11 \mathrm{mg} / \mathrm{L})$ and USGS $98(8.6 \mathrm{mg} / \mathrm{L})$.

\section{Total Recoverable Trace Elements}

Round-three water samples were analyzed for concentrations of total recoverable chromium, iron, lead, mercury, nickel, and silver (table 5). Statistical parameters for these constituents are provided by well in table 6 and were calculated using the data presented in this report.

The concentrations of all constituents in the blank sample (QAS-23) were less than the laboratory reporting levels. Concentrations of total recoverable chromium, mercury, nickel, and silver in the equipment blank sample (QAS-30) were less than the reporting levels; however, iron $(110 \mu \mathrm{g} / \mathrm{L})$ and lead $(17 \mu \mathrm{g} / \mathrm{L})$ concentrations were larger than the laboratory reporting levels of 10 and $1 \mu \mathrm{g} / \mathrm{L}$, respectively. Ninety-five of 101 replicate-sample concentra- 
tions listed in table 5 were statistically equivalent to the primary-sample concentrations. The primary and replicate nickel samples from USGS 97 collected on November 4, 1993, were ruined and no analysis was completed. The iron concentrations of six replicate samples were not statistically equivalent to the iron concentrations in the primary water samples: QAS-18 $(240 \mu \mathrm{g} / \mathrm{L})$ and NRF-1 $(470 \mu \mathrm{g} / \mathrm{L})$; QAS-16 $(120 \mu \mathrm{g} / \mathrm{L})$ and NRF-3 $(60 \mu \mathrm{g} / \mathrm{L})$; QAS-26 $(100 \mu \mathrm{g} / \mathrm{L})$ and NRF-3 $(320 \mu \mathrm{g} / \mathrm{L})$; QAS-17 $(90 \mu \mathrm{g} / \mathrm{L})$ and USGS 12 $(50 \mu \mathrm{g} / \mathrm{L}) ; \mathrm{QAS}-33(270 \mu \mathrm{g} / \mathrm{L})$ and USGS 12 $(140 \mu \mathrm{g} / \mathrm{L}) ;$ and QAS-32 $(390 \mu \mathrm{g} / \mathrm{L})$ and USGS $97(730 \mu \mathrm{g} / \mathrm{L})$.

\section{Dissolved Nutrients}

Filtered round-three water samples were analyzed for concentrations of either ammonia as nitrogen or ammonia plus organic nitrogen as nitrogen, nitrite as nitrogen, nitrite plus nitrate as nitrogen, and orthophosphate as phosphorus (table 7). Beginning with the December 1991 sample collection round, the NWQL was requested to analyze water samples for ammonia as nitrogen. Prior to that sample round, the laboratory had been requested to analyze water samples for ammonia plus organic nitrogen as nitrogen. Because of the difference in the two categories of ammonia compounds, the analytical results are not directly comparable. Statistical parameters for these constituents are provided by well in table 8 and were calculated using the data presented in this report.

Concentrations of all constituents in the blank sample (QAS-23) were less than the laboratory reporting levels. In the equipment blank sample (QAS-30), all constituents except for ammonia as nitrogen were less than the laboratory reporting levels. The concentration of ammonia as nitrogen in QAS-30 was $0.02 \mathrm{mg} / \mathrm{L}$ compared to the laboratory reporting level of $0.01 \mathrm{mg} / \mathrm{L}$. Sixty-three of 68 replicate-sample concentrations for nitrogen and phosphorus compounds listed in table 7 were statistically equivalent to their primarysample concentrations. The orthophosphate as phosphorus concentrations in QAS-15 (0.01 $\mathrm{mg} / \mathrm{L})$, QAS-22 (0.02 mg/L), QAS-29 (0.02 $\mathrm{mg} / \mathrm{L})$, and QAS-33 (0.02 $\mathrm{mg} / \mathrm{L})$ were not statistically equivalent to the concentrations in the respective primary water samples, USGS $97(0.02 \mathrm{mg} / \mathrm{L})$, USGS $15(0.01 \mathrm{mg} / \mathrm{L})$, USGS $17(0.03 \mathrm{mg} / \mathrm{L})$, and USGS $12(0.03 \mathrm{mg} / \mathrm{L})$. The statistical equivalence of the ammonia plus organic nitrogen (as nitrogen) concentration in the replicate water sample (QAS-13) and the primary water sample (NRF-2) was uncertain.

\section{Total Organic Carbon}

Round-three water samples were analyzed for concentrations of TOC (table 7). Statistical parameters for TOC are provided by well in table 8 and were calculated using the data presented in this report.

The concentration of TOC in the blank sample (QAS-23) was less than the laboratory reporting level of $0.1 \mathrm{mg} / \mathrm{L}$ and TOC analysis was not requested for the equipment blank sample (QAS-30). Fourteen of 17 replicatesample TOC concentrations listed in table 7 are statistically equivalent to their primarysample concentrations. The TOC concentrations of QAS-31 (1.8 mg/L), QAS-16 $(0.5 \mathrm{mg} / \mathrm{L})$, and QAS-28 $(0.5 \mathrm{mg} / \mathrm{L})$ were not statistically equivalent to the concentrations in their respective primary water samples, NRF-2 $(0.5 \mathrm{mg} / \mathrm{L})$, NRF-3 (1.8 mg/L), and NRF-4 $(1.0 \mathrm{mg} / \mathrm{L})$.

\section{Gross Alpha- and Gross Beta-Particle Radioactivity}

Round-three water samples were analyzed for concentrations of both dissolved and suspended gross alpha- and gross beta-particle 
radioactivity prior to the June 1993 sampling round. Beginning with the June 1993 sample round, analysis of the suspended fraction of water samples for gross alpha- and gross betaparticle radioactivity was discontinued because of potential precipitation of radionuclides onto suspended material or the container walls (Ann Mullin, U.S. Geological Survey, oral commun., 1995). Concentrations were determined by the laboratory using a residue procedure.

Concentrations of radioactive constituents that are greater than or equal to 3 times the $1 \mathrm{~s}$ uncertainty are considered to be above the reporting level in this report; however, all analytical measurements are listed in tables 9 and 10. Concentrations which meet or exceed the reporting level are shown in boldface type. For a more detailed discussion of reporting levels for radioactive constituents and measurements see the section of this report titled "Guidelines for Interpreting Results of Radiochemical Analyses".

Gross alpha-particle radioactivity.-Gross alpha-particle radioactivity is a measure of the total radioactivity given off as alpha particles during the radioactive decay process. For convenience, laboratories report the radioactivity as if it all were given off by one radionuclide. In this report, concentrations are reported two ways: as natural uranium in micrograms per liter and as thorium-230 in picocuries per liter. Concentrations of both dissolved and suspended gross alpha-particle radioactivity are listed in table 9 . Statistical parameters are provided by well in table 11 and were calculated using the data presented in this report.

Concentrations of gross alpha-particle radioactivity in the blank sample (QAS-23) and the equipment blank (QAS-30) were less than the reporting levels. Fifty-three of 60 replicate-sample concentrations listed in table 9 were statistically equivalent to their primary- sample concentration. The concentration as dissolved uranium in QAS-25 (2.97 $\pm \mathbf{0 . 5 2}$ $\mu \mathrm{g} / \mathrm{L})$ was not statistically equivalent to the concentration in the primary sample, USGS 98 $(1.49 \pm 0.356 \mu \mathrm{g} / \mathrm{L})$. The concentrations as dissolved thorium-230 in QAS-32 (3.79 10.96 $\mathrm{pCi} / \mathrm{L})$ and $\mathrm{QAS}-25(\mathbf{2 . 1 3} \pm \mathbf{0 . 3 7 7} \mathrm{pCi} / \mathrm{L})$ also were not statistically equivalent to the concentrations in the respective primary water samples, USGS $97(1.70 \pm 0.66 \mathrm{pCi} / \mathrm{L})$ and USGS 98 (1.02 $\pm \mathbf{0 . 2 4 2} \mathrm{pCi} / \mathrm{L})$. Concentrations as suspended uranium in QAS-13 $(-0.122 \pm 0.118 \mu \mathrm{g} / \mathrm{L})$ and QAS-26 $(0.122 \pm 0.168 \mu \mathrm{g} / \mathrm{L})$ were not statistically equivalent to the concentrations in the respective primary water samples, NRF-2 $(0.539 \pm 0.298 \mu \mathrm{g} / \mathrm{L})$ and NRF-3 (-0.348 \pm 0.164 $\mu \mathrm{g} / \mathrm{L})$. Finally, the concentrations as suspended thorium-230 in QAS-13 (-0.066 \pm 0.064 $\mathrm{pCi} / \mathrm{L})$ and QAS-26 (0.066 $\pm 0.092 \mathrm{pCi} / \mathrm{L})$ were not statistically equivalent to the concentraions in the respective primary water samples, NRF-2 (0.291 $\pm 0.164 \mathrm{pCi} / \mathrm{L})$ and NRF-3 $(-0.200 \pm 0.096 \mathrm{pCi} / \mathrm{L})$.

Gross beta-particle radioactivity.-Gross beta-particle radioactivity is a measure of the total radioactivity given off as beta particles during the radioactive decay process. For convenience, laboratories report the radioactivity as if it all were given off by one radionuclide or a chemically similar pair of radionuclides in equilibrium. In this report, concentrations are reported in two ways: as strontium-90 in equilibrium with yttrium-90 $\left(\mathrm{Sr}^{90} / \mathrm{Y}^{90}\right)$ in picocuries per liter, and as cesium-137 in picocuries per liter. Concentrations of both dissolved and suspended gross beta-particle radioactivity are listed in table 10. Statistical parameters are provided by well in table 11 and were calculated using the data presented in this report.

Concentrations of gross beta-particle radioactivity in the blank sample (QAS-23) and the equipment blank (QAS-30) were less than the reporting levels. All 60 replicate- 
sample concentrations listed in table 10 were statistically equivalent to their primary-sample concentration.

\section{ANALYTICAL RESULTS FOR ROUND- TWO WATER SAMPLES}

During the period beginning in November 1991 and ending in July 1992, five sets of bimonthly water samples were collected for round two of the NRF sampling program. Two wells (NRF-6 and NRF-7) were sampled all five times (table 2). Two replicate samples were collected during this period; NRF-6 (QAS-21) and NRF-7 (QAS-20). The roundtwo data in this report completes publication of analytical results for samples collected during the bimonthly sampling program. Previous round-two analytical results for NRF-1, $-2,-3$, -4 , USGS 12, 15, 17, 97, 98, 99, 102, and Water Supply INEL-1 and for associated quality-assurance samples were published by Bartholomay and others (1993).

\section{Selected Inorganic Constituents}

Round-two water samples were analyzed for concentrations of dissolved bromide, chloride, fluoride, sulfate, ammonia as nitrogen, nitrite as nitrogen, nitrite plus nitrate as nitrogen, and orthophosphate as phosphorus, and for concentrations of total recoverable sodium, arsenic, barium, cadmium, chromium, copper, iron, lead, manganese, mercury, nickel, selenium, silver, and zinc (table 12). Statistical parameters for these constituents are provided by well in tables 4,6 , and 8 and were calculated using the data presented in this report. Forty-two of 43 replicate-sample concentrations listed in table 12 were statistically equivalent to the primarysample concentrations. The iron concentration of QAS-21 $(80 \mu \mathrm{g} / \mathrm{L})$ was not statistically equivalent to the concentration in the primary water sample, NRF-6 $(120 \mu \mathrm{g} / \mathrm{L})$. The January
1992 replicate-water sample (QAS-20) and the corresponding primary-water sample (NRF-7) for determinations of total recoverable copper were ruined by the laboratory.

\section{Total Organic Carbon and Phenols}

Round-two water samples were analyzed for TOC concentrations and total phenol concentrations (table 13). Statistical parameters are provided by well in table 8 and were calculated using the data presented in this report. Three of four replicate-sample concentrations for these constituents listed in table 13 were statistically equivalent to the primarysample concentrations. The statistical equivalence of the total phenol concentration in the replicate-water sample (QAS-20) and the primary-water sample (NRF-7) was uncertain.

\section{Turbidity}

Round-two water samples were analyzed for turbidity (table 13). Statistical parameters are provided by well in table 8 and were calculated using the data presented in this report. The two replicate-sample measurements listed in table 13, 0.6 nephelometric turbidity units (NTU) for QAS-21 and 1.9 NTU for QAS-20, are similar to measurements for the respective primary-water samples, 0.5 NTU for NRF-6 and 2.0 NTU for NRF-7.

\section{Extractable Acid and Base/Neutral Organic Compounds}

Round-two primary- and replicate-water samples were analyzed by the NWQL for 57 extractable acid and base/neutral organic compounds (table 14). None of the 57 compounds were detected at concentrations greater than laboratory reporting levels (table 14). Analytical results for extractable acid and base/neutral compounds listed in table 14 have 
been confirmed by direct comparison with reference standards. Therefore, compound identification is positive, and reported concentrations are quantitative. The compounds listed in table 15 for the January 1992 NRF-7 sample are tentatively identified organic compounds (TIOC's ${ }^{1}$ ). The replicate sample (QAS-20) of the January 1992 NRF-7 sample did not contain concentrations of TIOC's.

\section{Selected Pesticides}

Round-two water samples were analyzed for concentrations of 15 organochlorine insecticides, gross polychlorinated biphenyls, gross polychlorinated naphthalenes, and 4 chlorophenoxy-acid herbicides (table 16). Pesticides were not detected in any round-two samples at concentrations larger than laboratory reporting levels.

\section{Gross Alpha- and Gross Beta-Particle Radioactivity}

Round-two water samples were analyzed for concentrations of both dissolved and suspended gross alpha- and gross beta-particle radioactivity using a residue procedure.

\section{Gross alpha-particle radioactivity.-} Concentrations of gross alpha-particle radioactivity for round-two water samples are reported two ways: as natural uranium in micrograms per liter and as thorium-230 in picocuries per liter. Concentrations of both dissolved and suspended gross alpha-particle radioactivity for round-two samples are listed in table 17. Statistical parameters for gross alpha-particle radioactivity are provided by

${ }^{1}$ Data for TIOC's in this report are based on comparison of sample spectra with library spectra followed by visual examination by gas chromatograph/mass spectrometer analysts. TIOC data have not been confirmed by direct comparison with reference standards. Therefore, TIOC identification is tentative, and reported concentrations are semiquantitative. well in table 11 and were calculated using the data presented in this report. All eight replicate-sample concentrations of gross alpha-particle radioactivity listed in table 17 were statistically equivalent to the respective primary-sample concentrations.

Gross beta-particle radioactivity.Concentrations of gross beta-particle radioactivity for round-two water samples are reported two ways: as strontium-90 in equilibrium with yttrium- $90\left(\mathrm{Sr}^{90} / \mathrm{Y}^{90}\right)$ in picocuries per liter and as cesium- 137 in picocuries per liter. Concentrations of both dissolved and suspended gross beta-particle radioactivity for round-two samples are listed in table 17. Statistical parameters are provided by well in table 11 and were calculated from the data presented in this report. All eight replicate-sample concentrations of gross betaparticle radioactivity listed in table 17 were statistically equivalent to the respective primary-sample concentrations.

\section{Selected Dissolved Radium Isotopes}

Round-two water samples were analyzed for dissolved concentrations of radium-226 by radon emanation and radium- 228 by separation and beta counting (table 17). Statistical parameters for radium-226 and radium-228 are provided by well in table 11 and were calculated using the data presented in this report. All four replicate-sample concentrations for radium isotopes listed in table 17 were statistically equivalent to the primary-sample concentrations.

\section{ANALYTICAL RESULTS FOR ROUND- ONE WATER SAMPLES}

\author{
During September 1991, water samples \\ were collected for the round-one NRF
} sampling program at wells NRF-6 and NRF-7 (table 2). Quality-assurance sampling for the round-one sampling program consisted of a 
blank sample (QAS-1) and a replicate sample (QAS-4); the analytical results for these samples were published by Knobel and others (1992). The data in this report completes publication of analytical results for samples collected during round one. Previous roundone analytical results for NRF-1, $-2,-3,-4$, USGS $12,15,17,97,98,99,102$, and Water Supply INEL-1 were published by Knobel and others (1992).

\section{Selected Inorganic Constituents}

Round-one water samples were analyzed for concentrations of dissolved bromide, chloride, fluoride, sulfate, thallium, cyanide, and orthophosphate as phosphorus, and for concentrations of total recoverable calcium, potassium, magnesium, sodium, aluminum, arsenic, barium, beryllium, cadmium, chromium, cobalt, copper, iron, lead, manganese, mercury, nickel, selenium, silver, zinc, ammonia as nitrogen, ammonia plus organic nitrogen as nitrogen, nitrite as nitrogen, nitrite plus nitrate as nitrogen, phosphorus, and orthophosphate as phosphorus (table 18). Statistical parameters for selected constituents are provided by well in tables 4,6 , and 8 and were calculated using the data presented in this report.

\section{Total Organic Carbon and Phenols}

Round-one water samples were analyzed for TOC concentrations and total phenol concentrations (table 19). Statistical parameters are provided in table 8 and were calculated using the data presented in this report.

\section{Total Recoverable Anionic Surfactants and Turbidity}

Round-one water samples were analyzed for total recoverable anionic surfactants reported as methylene blue active substances and measured for turbidity as NTU's (table 19). Statistical parameters for turbidity are provided in table 8 and were calculated using the data presented in this report. Data were not sufficient to calculate statistical parameters for anionic surfactants.

\section{Extractable Acid and Base/Neutral Organic Compounds}

Round-one water samples were analyzed for 57 extractable acid and base/neutral organic compounds (table 14). None of the 57 compounds were detected at concentrations greater than the respective laboratory reporting levels (table 14). In addition, no TIOC's were detected in the water samples.

\section{Purgeable Organic Compounds}

Round-one water samples (table 2) were analyzed for 36 purgeable organic compounds (POC's) which are listed in table 20. The sample from NRF- 6 contained $0.4 \mu \mathrm{g} / \mathrm{L}$ of chloroform, $0.5 \mu \mathrm{g} / \mathrm{L}$ of tetrachloroethylene, and $0.6 \mu \mathrm{g} / \mathrm{L}$ of toluene; the sample from NRF-7 contained $0.4 \mu \mathrm{g} / \mathrm{L}$ of $1,1,1$ trichloroethane. Other POC's listed in table 20 were not detected at concentrations larger than the respective laboratory reporting levels.

\section{Pesticides}

Round-one water samples were analyzed for concentrations of 24 triazine herbicides, 4 chlorophenoxy-acid herbicides, 12 carbamate insecticides, 11 organophosphorus insecticides, 15 organochlorine insecticides, 3 benzene hexachlorides, gross polychlorinated biphenyls, gross polychlorinated naphthalenes, and 7 aroclors (tables 21-22). Pesticides were not detected in any round-one samples at concentrations larger than the laboratory reporting levels. 


\section{Gross Alpha- and Gross Beta-Particle Radioactivity}

Round-one water samples were analyzed for concentrations of both dissolved and suspended gross alpha- and gross beta-particle radioactivity using a residue procedure.

Gross alpha-particle radioactivity.Concentrations of gross alpha-particle radioactivity for round-one water samples are reported two ways: as natural uranium in micrograms per liter and as thorium-230 in picocuries per liter. Concentrations of both dissolved and suspended gross alpha-particle radioactivity for round-one samples are listed in table 23. Statistical parameters for gross alpha-particle radioactivity are provided by well in table 11 and were calculated using the data presented in this report.

Gross beta-particle radioactivity.Concentrations of gross beta-particle radioactivity for round-one water samples are reported two ways: as strontium-90 in equilibrium with yttrium-90 $\left(\mathrm{Sr}^{90} / \mathrm{Y}^{90}\right)$ in picocuries per liter, and as cesium-137 in picocuries per liter. Concentrations of both dissolved and suspended gross beta-particle radioactivity for round-one samples are listed in table 23. Statistical parameters are provided by well in table 11 and were calculated using the data presented in this report.

\section{Selected Dissolved Radium Isotopes and Tritium}

Round-one water samples were analyzed for dissolved concentrations of radium- 226 by radon emanation and radium- 228 by separation and beta counting, and for total tritium by liquid scintillation (table 23). Statistical parameters for radium-226 and radium-228 are provided by well in table 11 and were calculated using the data presented in this report. Data were not sufficient to calculate statistical parameters for tritium.

\section{SUMMARY}

The USGS, in response to a request from the U.S. Department of Energy's Pittsburgh Naval Reactors Office, Idaho Branch Office, sampled 14 wells during 1991-93 as part of a long-term project to monitor water quality of the Snake River Plain aquifer in the vicinity of the NRF, INEL, Idaho. Water samples were collected and analyzed for manmade contaminants and naturally occurring constituents. One hundred sixty-one samples were collected from 10 ground-water monitoring wells and 4 production wells. Twenty-one qualityassurance samples also were collected and analyzed; 2 blank samples and 19 replicate samples. The two quality-assurance blank samples contained larger concentrations than the laboratory reporting levels for bromide, chloride, iron, lead, sodium, and ammonia as nitrogen. Concentrations of other constituents in the blank samples were less than the respective laboratory reporting levels. The 19 replicate samples and their respective primary samples generated 614 pairs of analytical results for a variety of chemical and radiochemical constituents. Of the 614 data pairs, 588 were statistically equivalent at the 95percent confidence level; about 96 percent of the analytical results were in agreement. Of the 24 data pairs not statistically equivalent, 1 was ammonia plus organic nitrogen as nitrogen, 1 was bromide, 4 were gross alpha-particle radioactivity as thorium, 3 were gross alphaparticle radioactivity as uranium, 7 were iron, 4 were orthophosphate as phosphorus, 1 was phenol, 2 were sodium, and 3 were TOC. In addition, two pairs of data for turbidity samples were not evaluated because of insufficient information and the sample collected from NRF-7 in January 1992 contained TIOC's when the replicate sample (QAS-20) did not. 
SELECTED REFERENCES

Ackerman, D.J., 1991, Transmissivity of the Snake River Plain aquifer at the Idaho National Engineering Laboratory, Idaho: U.S. Geological Survey Water-Resources Investigations Report 91-4058 (DOE/ID22097), $35 \mathrm{p}$.

American Society for Testing and Materials, 1992, ASTM standards on precision and bias for various applications: Philadelphia, Pennsylvania, American Society for Testing and Materials Publication code number (PCN) 03-511092-34, 478 p.

Bartholomay, R.C., Knobel, L.L., and Tucker, B.J., 1993, Chemical constituents in water from wells in the vicinity of the Naval Reactors Facility, Idaho National Engineering Laboratory, Idaho, 1990-91: U.S. Geological Survey Open-File Report 9394 (DOE/ID-22106), 70 p.

Bettis Atomic Power Laboratory, 1992, Naval Reactors Facility Site Environmental Summary Report: Westinghouse Electric Corporation NRFRC-EC-1046, 26 p.

Claassen, H.C., 1982, Guidelines and techniques for obtaining water samples that accurately represent the water chemistry of an aquifer: U.S. Geological Survey Open-File Report 82-1024, 49 p.

Currie, L.A., 1984, Lower limit of detection-Definition and elaboration of a proposed position for radiological effluent and environmental measurements: U.S. Nuclear Regulatory Commission NUREG/CR-4077, 139 p.

Faires, L.M., 1992, Methods of analysis by the U.S. Geological Survey National Water Quality Laboratory-Determination of metals in water by inductively coupled plasma-mass spectrometry: U.S. Geological Survey Open-File Report 92634, $28 \mathrm{p}$.
Fishman, M.J., ed., 1993, Methods of analysis by the U.S. Geological Survey National Water Quality Laboratory - Determination of inorganic and organic constituents in water and fluvial sediments: U.S. Geological Survey Open-File Report 93$125,217 \mathrm{p}$.

Fishman, M.J., and Friedman, L.C., eds., 1989, Methods for determination of inorganic substances in water and fluvial sediments: U.S. Geological Survey Techniques of Water-Resources Investigations, book 5, chap. A1, 545 p.

Friedman, L.C., and Erdmann, D.E., 1982, Quality assurance practices for the chemical and biological analyses of water and fluvial sediments: U.S. Geological Survey Techniques of Water-Resources Investigations, book 5, chap. A6, 181 p.

Goerlitz, D.F., and Brown, Eugene, 1972, Methods for analysis of organic substances in water: U.S. Geological Survey Techniques of Water-Resources Investigations, book 5, chap. A3, 40 p.

Hardy, M.A., Leahy, P.P., and Alky, W.M., 1989, Well installation and documentation, and ground-water sampling protocols for the pilot national water-quality assessment program: U.S. Geological Survey Open-File Report 89-396, 36 p.

Iman, R.L., and Conover, W.J., 1983, A modern approach to statistics: New York, John Wiley \& Sons, Inc., 497 p.

Jones, B.E., 1987, Quality control manual of the U.S. Geological Survey's National Water Quality Laboratory: U.S. Geological Survey Open-File Report 87-457, $17 \mathrm{p}$. 
Knobel, L.L., Bartholomay, R.C., Wegner, S.J., and Edwards, D.D., 1992, Chemical constituents in water from wells in the vicinity of the Naval Reactors Facility, Idaho National Engineering Laboratory, Idaho, 1989-90: U.S. Geological Survey Open-File Report 92-156 (DOE/ID-22103), 38 p.

Knobel, L.L., Orr, B.R., and Cecil, L.D., 1992, Summary of background concentrations of selected radiochemical and chemical constituents in groundwater from the Snake River Plain aquifer, Idaho: estimated from an analysis of previously published data: Journal of the Idaho Academy of Science, v. 28, no. 1, p. 4861.

Maloney, T.J., Ludtke, A.S., Krizman, T.L., 1993, Quality-assurance data for routine water analysis in the laboratories of the U.S. Geological Survey for water year 1990: U.S. Geological Survey WaterResources Investigations Report 93-4082, $145 \mathrm{p}$.

Mann, L.J., 1986, Hydraulic properties of rock units and chemical quality of water for INEL-1-a 10,365-foot deep test hole drilled at the Idaho National Engineering Laboratory, Idaho: U.S. Geological Survey Water-Resources Investigations Report 86-4020 (DOE/ID-22070), 23 p.

----- 1989, Tritium concentrations in flow from selected springs that discharge to the Snake River, Twin Falls-Hagerman area, Idaho: U.S. Geological Survey WaterResources Investigations Report 89-4156 (DOE/ID-22084), $20 \mathrm{p}$.

Orr, B.R., and Cecil, L.D., 1991, Hydrologic conditions and distribution of selected chemical constituents in water, Snake River Plain aquifer, Idaho National
Engineering Laboratory, Idaho, 1986 to 1988: U.S. Geological Survey WaterResources Investigations Report 91-4047 (DOE/ID-22096), $56 \mathrm{p}$.

Pritt, J.W., 1989, Quality assurance of sample containers and preservatives at the U.S. Geological Survey National Water Quality Laboratory, in Pederson, G.L., and Smith, M.M., compilers, U.S. Geological Survey Second National Symposium on Water Quality-Abstracts of the Technical Sessions, Orlando, Fla., November 12-17, 1989: U.S. Geological Survey Open-File Report 89-409, p. 75.

Pritt, Jeffrey, and Jones, B.E., eds., 1989, 1990 National Water Quality Laboratory services catalog: U.S. Geological Survey Open-File Report 89-386, unnumbered.

Pritt, J.W., and Raese, J.W., eds., 1992, Quality assurance/quality control manual, National Water Quality Laboratory: U.S. Geological Survey Open-File Report 92495, $33 \mathrm{p}$.

Stevens, H.H., Jr., Ficke, J.F., and Smoot, G.F., 1975, Water temperatureinfluential factors, field measurement, and data presentation: U.S. Geological Survey Techniques of Water-Resources Investigations, book 5, chap. D1, 65 p.

Taylor, J.K., 1987, Quality assurance of chemical measurements: Chelsea, Michigan, Lewis Publishers, Inc., 328 p.

Thatcher, L.L., Janzer, V.J., and Edwards, K.W., 1977, Methods for determination of radioactive substances in water and fluvial sediments: U.S. Geological Survey Techniques of Water-Resources Investigations, book 5, chap. A5, $95 \mathrm{p}$. 
U.S. Environmental Protection Agency, 1989, Protection of Environment, Code of Federal Regulations 40: Office of the Federal Register, National Archives and Records Administration, pts. 190 to 299, $1,027 \mathrm{p}$.

U.S. Geological Survey, 1985, National water summary, 1984-Hydrologic events, selected water-quality trends, and groundwater resources: U.S. Geological Survey Water-Supply Paper 2275, 467 p.

Volk, William, 1969, Applied statistics for engineers ( $2 \mathrm{~d}$ ed.): New York, McGrawHill Book Company, $415 \mathrm{p}$.

Wegner, S.J., 1989, Selected water quality assurance data for water samples collected by the U.S. Geological Survey, Idaho National Engineering Laboratory, Idaho, 1980 to 1988: U.S. Geological Survey Water-Resources Investigations Report 89-4168 (DOE/ID-22085), 91 p.

Wershaw, R.L., Fishman, M.J., Grabbe, R.R., and Lowe, L.E., 1987, Method for the determination of organic substances in water and fluvial sediments: U.S. Geological Survey Techniques of WaterResources Investigations, book 5, chap. A3, $80 \mathrm{p}$.

Wood, W.W., 1981, Guidelines for collection and field analysis of ground-water samples for selected unstable constituents: U.S. Geological Survey Techniques of Water-Resources Investigations, book 1 , chap. D2, 24 p. 
Table 1.-Containers and preservatives used for water samples, Naval Reactors Facility and vicinity :-

[Analyses by U.S. Geological Survey's National Water Quality Laboratory. Abbreviations: L, liter; mL, milliliter; $\mathrm{H}_{3} \mathrm{PO}_{4}$, phosphoric acid; $\mathrm{CuSO}_{4}$, copper sulfate; $\mathrm{HgCl}_{2}$, mercuric chloride; $\mathrm{NaCl}$, sodium chloride; $\mathrm{HNO}_{3}$, nitric acid; $\mathrm{K}_{2} \mathrm{Cr}_{2} \mathrm{O}_{7}$, potassium dichromate; $\mathrm{HCl}$, hydrochloric acid; $\mathrm{NaOH}$, sodium hydroxide; ${ }^{\circ} \mathrm{C}$, degrees Celsius. Teflon: The use of trade names in this report is for identification purposes only and does not constitute endorsement by the U.S. Geological Survey. Samples were shipped by overnight-delivery mail]

\begin{tabular}{|c|c|c|c|c|c|}
\hline \multirow{2}{*}{ Type of constituent } & \multicolumn{2}{|c|}{ Container } & \multicolumn{2}{|c|}{ Preservative } & \multirow{2}{*}{$\begin{array}{l}\text { Other } \\
\text { treatment }\end{array}$} \\
\hline & Type & Size & Type & Volume & \\
\hline Anions & Polyethylene & $250 \mathrm{~mL}$ & None & None & Filter \\
\hline Cations, total recoverable & $\begin{array}{l}\text { Polyethylene, } \\
\text { acid-rinsed }\end{array}$ & $250 \mathrm{~mL}$ & $\mathrm{HNO}_{3}$ & $1 \mathrm{~mL}$ & None \\
\hline \multirow[t]{3}{*}{$\begin{array}{l}\text { Trace elements, total } \\
\text { recoverable }\end{array}$} & $\begin{array}{l}\text { Polyethylene, } \\
\text { acid-rinsed }\end{array}$ & $1 \mathrm{~L}$ & $\mathrm{HNO}_{3}$ & $4 \mathrm{~mL}$ & None \\
\hline & $\begin{array}{l}\text { Polyethylene, } \\
\text { acid-rinsed }\end{array}$ & $500 \mathrm{~mL}$ & $\mathrm{HNO}_{3}$ & $2 \mathrm{~mL}$ & None \\
\hline & $\begin{array}{l}\text { Polyethylene, } \\
\text { acid-rinsed }\end{array}$ & $250 \mathrm{~mL}$ & $\mathrm{HNO}_{3}$ & $1 \mathrm{~mL}$ & None \\
\hline Mercury, total recoverable & $\begin{array}{l}\text { Glass, } \\
\text { acid-rinsed }\end{array}$ & $250 \mathrm{~mL}$ & $\mathrm{~K}_{2} \mathrm{Cr}_{2} \mathrm{O}_{7} / \mathrm{HNO}_{3}$ & $10 \mathrm{~mL}$ & None \\
\hline Thallium, dissolved & $\begin{array}{l}\text { Teflon, } \\
\text { acid-rinsed }\end{array}$ & $250 \mathrm{~mL}$ & $\mathrm{HNO}_{3}$, ultrapure & $1 \mathrm{~mL}$ & Filter \\
\hline Nutrients, total recoverable & $\begin{array}{l}\text { Polyethylene, } \\
\text { brown }\end{array}$ & $250 \mathrm{~mL}$ & $\mathrm{HgCl}_{2} / \mathrm{NaCl}$ & $1 \mathrm{~mL}$ & Chill $4{ }^{\circ} \mathrm{C}$ \\
\hline Nutrients, dissolved & $\begin{array}{l}\text { Polyethylene, } \\
\text { brown }\end{array}$ & $250 \mathrm{~mL}$ & $\mathrm{HgCl}_{2} / \mathrm{NaCl}$ & $1 \mathrm{~mL}$ & Filter, chill $4^{\circ} \mathrm{C}$ \\
\hline Nutrients, dissolved & $\begin{array}{l}\text { Polyethylene, } \\
\text { brown }\end{array}$ & $125 \mathrm{~mL}$ & $\mathrm{HgCl}_{2} / \mathrm{NaCl}$ & $.5 \mathrm{~mL}$ & Filter, chill $4^{\circ} \mathrm{C}$ \\
\hline Cyanide & Polyethylene & $250 \mathrm{~mL}$ & $\mathrm{NaOH}$ & $5 \mathrm{~mL}$ & Chill $4^{\circ} \mathrm{C}$ \\
\hline Total organic carbon & Glass, baked & $125 \mathrm{~mL}$ & None & None & Chill $4^{\circ} \mathrm{C}$ \\
\hline Anionic surfactants & Polyethylene & $250 \mathrm{~mL}$ & None & None & Chill $4^{\circ} \mathrm{C}$ \\
\hline Turbidity & Polyethylene & $125 \mathrm{~mL}$ & None & None & None \\
\hline Purgeable organic compounds & Glass, baked & $40 \mathrm{~mL}$ & None & None & Chill $4^{\circ} \mathrm{C}$ \\
\hline $\begin{array}{l}\text { Extractable acid and base/ } \\
\text { neutral organic compounds }\end{array}$ & Glass, baked & $1 \mathrm{~L}$ & None & None & Chill $4^{\circ} \mathrm{C}$ \\
\hline Phenols, total & Glass, baked & $1 \mathrm{~L}$ & $\mathrm{H}_{3} \mathrm{PO}_{4} / \mathrm{CuSO}_{4}$ & $10 \mathrm{~mL}$ & Chill $4^{\circ} \mathrm{C}$ \\
\hline
\end{tabular}


Table 1.-Containers and preservatives used for water samples, Naval Reactors Facility and vicinity-Continued

\begin{tabular}{|c|c|c|c|c|c|}
\hline \multirow{2}{*}{ Type of constituent } & \multicolumn{2}{|c|}{ Container } & \multicolumn{2}{|c|}{ Preservative } & \multirow{2}{*}{$\begin{array}{c}\text { Other } \\
\text { treatment }\end{array}$} \\
\hline & Type & Size & Type & Volume & \\
\hline Pesticides & Glass, baked & $1 \mathrm{~L}$ & None & None & Chill $4^{\circ} \mathrm{C}$ \\
\hline Gross alpha and beta & $\begin{array}{l}\text { Polyethylene, } \\
\text { acid-rinsed }\end{array}$ & $1 \mathrm{~L}$ & None & None & None \\
\hline Gross alpha and beta & $\begin{array}{l}\text { Polyethylene, } \\
\text { acid-rinsed }\end{array}$ & $1 \mathrm{~L}$ & $\mathrm{HNO}_{3}$ & $4 \mathrm{~mL}$ & Filter \\
\hline Tritium & $\begin{array}{l}\text { Polyethylene, } \\
\text { acid-rinsed }\end{array}$ & $250 \mathrm{~mL}$ & None & None & None \\
\hline Radium-226 & $\begin{array}{l}\text { Polyethylene, } \\
\text { acid-rinsed }\end{array}$ & $1 \mathrm{~L}$ & $\mathrm{HCl}$ & $5 \mathrm{~mL}$ & Filter \\
\hline Radium-228 & $\begin{array}{l}\text { Polyethylene, } \\
\text { acid-rinsed }\end{array}$ & $1 \mathrm{~L}$ & $\mathrm{HCl}$ & $5 \mathrm{~mL}$ & Filter \\
\hline
\end{tabular}


Table 2.- Results of field measurements for $p H$, specific conductance, and temperature of water, Naval Reactors Facility and vicinity

[Units: $\mathrm{pH}$, negative base-10 logarithm of hydrogen ion activity in moles per liter; specific conductance, microsiemens per centimeter at $25^{\circ} \mathrm{C}$ (degrees Celsius); temperature, ${ }^{\circ} \mathrm{C}$. Sample identifier: see figure 2 for location of wells; Blank - indicates sample was organic free water from the U.S. Geological Survey's National Water Quality Laboratory (NWQL); Equipment blank - sample was inorganic free water from the NWQL. Date sampled: $(\mathrm{m} / \mathrm{d} / \mathrm{y})$ indicates month/day/year of sample collection. Remarks: QAS indicates quality-assurance sample (values for field measurements for each pair of primary and replicate samples are the same measurement); data are from round three unless otherwise indicated. Symbol: NM indicates not measured]

\begin{tabular}{|c|c|c|c|c|c|c|}
\hline Sample identifier & $\begin{array}{l}\text { Date sampled } \\
\qquad(\mathrm{m} / \mathrm{d} / \mathrm{y})\end{array}$ & Time & $\mathrm{pH}$ & $\begin{array}{c}\text { Specific } \\
\text { conductance }\end{array}$ & Temperature & Remarks \\
\hline Blank & $6 / 12 / 92$ & 1030 & 8.9 & 9 & 22.0 & QAS-23 \\
\hline Equipment blank & $6 / 15 / 93$ & 1300 & 6.4 & 1 & 22.5 & QAS-30 \\
\hline \multirow[t]{13}{*}{ NRF-1 } & $3 / 5 / 91$ & 1125 & 8.1 & 585 & 12.5 & \\
\hline & $6 / 17 / 91$ & 1135 & 8.0 & 572 & 13.0 & \\
\hline & 9/9/91 & 1144 & 8.0 & 578 & 12.0 & \\
\hline & $9 / 9 / 91$ & 1100 & 8.0 & 578 & 12.0 & QAS-18, replicate \\
\hline & $12 / 4 / 91$ & 0940 & 8.0 & 559 & 12.0 & \\
\hline & $3 / 11 / 92$ & 1105 & 8.0 & 565 & 12.0 & \\
\hline & $6 / 11 / 92$ & 1235 & 8.0 & 590 & 12.5 & \\
\hline & $9 / 16 / 92$ & 1120 & 8.0 & 578 & 12.5 & \\
\hline & $12 / 3 / 92$ & 1045 & 8.0 & 579 & 12.0 & \\
\hline & $4 / 7 / 93$ & 1020 & 7.9 & 578 & 12.0 & \\
\hline & $6 / 15 / 93$ & 1012 & 8.0 & 598 & 12.0 & \\
\hline & $9 / 15 / 93$ & 1015 & 7.9 & 604 & 11.0 & \\
\hline & $11 / 4 / 93$ & 0915 & 7.8 & 600 & 12.0 & \\
\hline \multirow[t]{7}{*}{ NRF-2 } & $3 / 5 / 91$ & 1145 & 8.2 & 645 & 13.0 & \\
\hline & $3 / 5 / 91$ & 0900 & 8.2 & 645 & 13.0 & QAS-13, replicate \\
\hline & $6 / 17 / 91$ & 1035 & 7.9 & 625 & 13.0 & \\
\hline & 9/9/91 & 1049 & 8.0 & 630 & 12.5 & \\
\hline & $12 / 4 / 91$ & 1033 & 8.0 & 630 & 13.0 & \\
\hline & $3 / 11 / 92$ & 1024 & 8.0 & 612 & 12.5 & \\
\hline & $6 / 11 / 92$ & 1008 & 8.0 & 638 & 13.0 & \\
\hline
\end{tabular}


Table 2.- Results of field measurements for $\mathrm{pH}$, specific conductance, and temperature of water, Naval Reactors Facility and vicinity-Continued

\begin{tabular}{|c|c|c|c|c|c|c|}
\hline Sample identifier & $\begin{array}{l}\text { Date sampled } \\
\qquad(\mathrm{m} / \mathrm{d} / \mathrm{y})\end{array}$ & Time & $\mathrm{pH}$ & $\begin{array}{c}\text { Specific } \\
\text { conductance }\end{array}$ & Temperature & Remarks \\
\hline \multirow[t]{7}{*}{ NRF-2 - cont. } & $9 / 16 / 92$ & 1038 & 8.0 & 624 & 12.5 & \\
\hline & $12 / 3 / 92$ & 1000 & 8.0 & 620 & 12.5 & \\
\hline & $4 / 7 / 93$ & 0927 & 7.9 & 632 & 14.0 & \\
\hline & $6 / 15 / 93$ & 1048 & 7.9 & 650 & 12.5 & \\
\hline & $9 / 15 / 93$ & 1050 & 7.9 & 664 & 12.0 & \\
\hline & $9 / 15 / 93$ & 1030 & 7.9 & 664 & 12.0 & QAS-31, replicate \\
\hline & $11 / 4 / 93$ & 1037 & 7.9 & 664 & 12.0 & \\
\hline \multirow[t]{13}{*}{ NRF-3 } & $6 / 17 / 91$ & 1120 & 7.9 & 575 & 12.0 & \\
\hline & $6 / 17 / 91$ & 1100 & 7.9 & 575 & 12.0 & QAS-16, replicate \\
\hline & $9 / 26 / 91$ & 1440 & 8.0 & 590 & 13.0 & \\
\hline & $12 / 4 / 91$ & 0923 & 7.9 & 558 & 11.5 & \\
\hline & $3 / 11 / 92$ & 1310 & 8.0 & 570 & 12.0 & \\
\hline & $6 / 11 / 92$ & 1304 & 8.0 & 588 & 12.0 & \\
\hline & $9 / 16 / 92$ & 1255 & 8.0 & 588 & 12.0 & \\
\hline & $12 / 3 / 92$ & 1123 & 8.0 & 572 & 11.5 & \\
\hline & $12 / 3 / 92$ & 1100 & 8.0 & 572 & 11.5 & QAS-26, replicate \\
\hline & $4 / 8 / 93$ & 0945 & NM & 602 & 11.5 & \\
\hline & $6 / 15 / 93$ & 0940 & 7.9 & 598 & 11.5 & \\
\hline & $9 / 15 / 93$ & 0945 & 7.8 & 609 & 10.0 & \\
\hline & $11 / 4 / 93$ & 1055 & 7.9 & 607 & 11.5 & \\
\hline \multirow[t]{8}{*}{ NRF-4 } & $3 / 5 / 91$ & 1040 & 8.0 & 630 & 12.0 & \\
\hline & $6 / 17 / 91$ & 1155 & 8.0 & 578 & 12.0 & \\
\hline & 9/9/91 & 0937 & 8.0 & 600 & 12.0 & \\
\hline & $12 / 4 / 91$ & 1209 & 8.0 & 602 & 12.0 & \\
\hline & $3 / 11 / 92$ & 1000 & 7.9 & 605 & 12.0 & \\
\hline & $6 / 11 / 92$ & 0910 & 8.0 & 600 & 12.0 & \\
\hline & $9 / 16 / 92$ & 1325 & 8.0 & 610 & 12.0 & \\
\hline & $12 / 3 / 92$ & 0929 & 7.8 & 605 & 11.5 & \\
\hline
\end{tabular}


Table 2.-Results of field measurements for $\mathrm{pH}$, specific conductance, and temperature of water, Naval Reactors Facility and vicinity-Continued

\begin{tabular}{|c|c|c|c|c|c|c|}
\hline Sample identifier & $\begin{array}{l}\text { Date sampled } \\
\qquad(\mathrm{m} / \mathrm{d} / \mathrm{y})\end{array}$ & Time & $\mathrm{pH}$ & $\begin{array}{c}\text { Specific } \\
\text { conductance }\end{array}$ & Temperature & Remarks \\
\hline \multirow[t]{5}{*}{ NRF-4 - cont. } & $4 / 7 / 93$ & 1122 & 7.9 & 610 & 12.0 & \\
\hline & $4 / 7 / 93$ & 1100 & 7.9 & 610 & 12.0 & QAS-28, replicate \\
\hline & $6 / 15 / 93$ & 0907 & 7.9 & 650 & 12.0 & \\
\hline & $9 / 15 / 93$ & 0905 & 7.8 & 662 & 11.0 & \\
\hline & $11 / 4 / 93$ & 0954 & 7.9 & 656 & 11.5 & \\
\hline \multirow[t]{12}{*}{ NRF-6 } & 9/9/91 & 1330 & 7.8 & 1,380 & 12.0 & Round one \\
\hline & $11 / 6 / 91$ & 1415 & 7.8 & 1,360 & 12.0 & Round two \\
\hline & $1 / 8 / 92$ & 1450 & 7.8 & 1,310 & 12.0 & Round two \\
\hline & $3 / 10 / 92$ & 1255 & 7.9 & 1,320 & 12.0 & Round two \\
\hline & $3 / 10 / 92$ & 1230 & 7.9 & 1,320 & 12.0 & $\begin{array}{l}\text { QAS- } 21 \text {, replicate, } \\
\text { round two }\end{array}$ \\
\hline & $5 / 14 / 92$ & 1320 & 7.9 & 1,340 & 12.0 & Round two \\
\hline & $7 / 8 / 92$ & 1445 & 7.9 & 1,390 & 12.5 & Round two \\
\hline & $9 / 18 / 92$ & 1515 & 8.0 & 1,400 & 12.0 & \\
\hline & $12 / 9 / 92$ & 1255 & 7.9 & 1,400 & 12.0 & \\
\hline & 4/9/93 & 1350 & 7.9 & 1,470 & 12.0 & \\
\hline & $9 / 14 / 93$ & 1400 & 7.8 & 1,450 & 11.5 & \\
\hline & $11 / 4 / 93$ & 1435 & 7.8 & 1,430 & 12.0 & \\
\hline \multirow[t]{10}{*}{ NRF-7 } & $9 / 10 / 91$ & 1300 & 8.5 & 254 & 15.5 & Round one \\
\hline & $11 / 6 / 91$ & 1245 & 8.5 & 242 & 15.0 & Round two \\
\hline & $1 / 8 / 92$ & 1310 & 8.5 & 232 & 15.0 & Round two \\
\hline & $1 / 8 / 92$ & 1400 & 8.5 & 232 & 15.0 & $\begin{array}{l}\text { QAS-20, replicate, } \\
\text { round two }\end{array}$ \\
\hline & $3 / 10 / 92$ & 1130 & 8.5 & 232 & 15.5 & Round two \\
\hline & $5 / 14 / 92$ & 1120 & 8.4 & 240 & 15.0 & Round two \\
\hline & $7 / 8 / 92$ & 1230 & 8.3 & 255 & 16.0 & Round two \\
\hline & $9 / 18 / 92$ & 1400 & 8.5 & 244 & 15.0 & \\
\hline & $12 / 9 / 92$ & 1140 & 8.3 & 243 & 15.0 & \\
\hline & $4 / 9 / 93$ & 1230 & 8.3 & 243 & 15.0 & \\
\hline
\end{tabular}


Table 2.- Results of field measurements for $\mathrm{pH}$, specific conductance, and temperature of water, Naval Reactors Facility and vicinity-Continued

\begin{tabular}{|c|c|c|c|c|c|c|}
\hline Sample identifier & $\begin{array}{l}\text { Date sampled } \\
\qquad(\mathrm{m} / \mathrm{d} / \mathrm{y})\end{array}$ & Time & $\mathrm{pH}$ & $\begin{array}{c}\text { Specific } \\
\text { conductance }\end{array}$ & Temperature & Remarks \\
\hline \multirow[t]{3}{*}{ NRF-7 - cont. } & $6 / 10 / 93$ & 1400 & 8.4 & 245 & 14.5 & \\
\hline & $9 / 14 / 93$ & 1215 & 8.1 & 254 & 14.0 & \\
\hline & $11 / 3 / 93$ & 1455 & 8.1 & 257 & 14.0 & \\
\hline \multirow[t]{13}{*}{ USGS 12} & $6 / 10 / 91$ & 1200 & 7.8 & 575 & 13.5 & \\
\hline & $9 / 6 / 91$ & 1505 & 7.9 & 590 & 12.5 & \\
\hline & $9 / 6 / 91$ & 1400 & 7.9 & 590 & 12.5 & QAS-17, replicate \\
\hline & $12 / 5 / 91$ & 1125 & 7.9 & 565 & 12.0 & \\
\hline & $3 / 12 / 92$ & 1520 & 7.8 & 610 & 12.5 & \\
\hline & $6 / 19 / 92$ & 1500 & 7.9 & 580 & 12.5 & \\
\hline & $9 / 18 / 92$ & 0945 & 8.0 & 560 & 12.0 & \\
\hline & $12 / 1 / 92$ & 1505 & 7.9 & 560 & 12.0 & \\
\hline & $4 / 13 / 93$ & 1450 & 7.8 & 582 & 12.0 & \\
\hline & $6 / 14 / 93$ & 1500 & 7.9 & 600 & 12.0 & \\
\hline & $9 / 16 / 93$ & 1455 & 7.8 & 580 & 11.5 & \\
\hline & $11 / 5 / 93$ & 1435 & 7.8 & 590 & 12.0 & \\
\hline & $11 / 5 / 93$ & 1400 & 7.8 & 590 & 12.0 & QAS-33, replicate \\
\hline \multirow[t]{12}{*}{ USGS 15} & $6 / 10 / 91$ & 1530 & 7.9 & 403 & 12.0 & \\
\hline & $9 / 6 / 91$ & 1240 & 7.9 & 495 & 11.5 & \\
\hline & $12 / 4 / 91$ & 1715 & 8.0 & 326 & 11.5 & \\
\hline & $3 / 12 / 92$ & 1240 & 8.1 & 315 & 12.0 & \\
\hline & $3 / 12 / 92$ & 1300 & 8.1 & 315 & 12.0 & QAS-22, replicate \\
\hline & $6 / 19 / 92$ & 1240 & 8.0 & 410 & 11.5 & \\
\hline & $9 / 17 / 92$ & 1445 & 8.0 & 415 & 12.0 & \\
\hline & $12 / 1 / 92$ & 1245 & 8.1 & 307 & 11.5 & \\
\hline & $4 / 13 / 93$ & 1230 & 8.0 & 298 & 12.0 & \\
\hline & $6 / 14 / 93$ & 1240 & 8.1 & 345 & 11.5 & \\
\hline & $9 / 16 / 93$ & 1235 & 7.8 & 421 & 11.0 & \\
\hline & $11 / 5 / 93$ & 1215 & 7.9 & 345 & 11.5 & \\
\hline
\end{tabular}


Table 2.-Results of field measurements for $\mathrm{pH}$, specific conductance, and temperature of water, Naval Reactors Facility and vicinity-Continued

\begin{tabular}{|c|c|c|c|c|c|c|}
\hline Sample identifier & $\begin{array}{l}\text { Date sampled } \\
\qquad(\mathrm{m} / \mathrm{d} / \mathrm{y})\end{array}$ & Time & $\mathrm{pH}$ & $\begin{array}{c}\text { Specific } \\
\text { conductance }\end{array}$ & Temperature & Remarks \\
\hline \multirow[t]{14}{*}{ USGS 17} & $3 / 13 / 91$ & 1145 & 8.2 & 292 & 13.0 & \\
\hline & $3 / 13 / 91$ & 1200 & 8.2 & 292 & 13.0 & \multirow[t]{10}{*}{ QAS-14, replicate } \\
\hline & $6 / 6 / 91$ & 1300 & 8.2 & 291 & 14.0 & \\
\hline & $9 / 5 / 91$ & 1150 & 8.2 & 299 & 13.5 & \\
\hline & $12 / 3 / 91$ & 1215 & 8.2 & 286 & 13.5 & \\
\hline & $3 / 16 / 92$ & 1140 & 8.2 & 285 & 13.5 & \\
\hline & $6 / 11 / 92$ & 1435 & 8.2 & 298 & 13.5 & \\
\hline & $9 / 16 / 92$ & 1445 & 8.2 & 295 & 13.5 & \\
\hline & $12 / 3 / 92$ & 1330 & 8.2 & 298 & 13.0 & \\
\hline & $4 / 7 / 93$ & 1310 & 8.2 & 289 & 13.5 & \\
\hline & $6 / 11 / 93$ & 1510 & 8.2 & 298 & 13.0 & \\
\hline & $6 / 11 / 93$ & 1500 & 8.2 & 298 & 13.0 & \multirow[t]{3}{*}{ QAS-29, replicate } \\
\hline & $9 / 15 / 93$ & 1225 & 8.1 & 298 & 12.5 & \\
\hline & $11 / 8 / 93$ & 1325 & 8.0 & 299 & 13.0 & \\
\hline \multirow[t]{14}{*}{ USGS 97} & $3 / 13 / 91$ & 1245 & 8.0 & 580 & 11.5 & \\
\hline & $6 / 7 / 91$ & 1050 & 8.0 & 582 & 12.0 & \\
\hline & $6 / 7 / 91$ & 1000 & 8.0 & 582 & 12.0 & \multirow[t]{11}{*}{ QAS-15, replicate } \\
\hline & $9 / 5 / 91$ & 1350 & 8.0 & 595 & 12.0 & \\
\hline & $12 / 3 / 91$ & 1425 & 8.0 & 579 & 11.5 & \\
\hline & $3 / 16 / 92$ & 1355 & 7.9 & 575 & 12.0 & \\
\hline & $6 / 17 / 92$ & 0950 & 7.9 & 572 & 12.0 & \\
\hline & $9 / 21 / 92$ & 1315 & 7.9 & 590 & 12.0 & \\
\hline & $12 / 8 / 92$ & 1530 & 8.0 & 557 & 12.0 & \\
\hline & $4 / 6 / 93$ & 1250 & 7.9 & 578 & 11.5 & \\
\hline & $6 / 9 / 93$ & 1125 & 8.0 & 585 & 11.5 & \\
\hline & $9 / 13 / 93$ & 1320 & 7.8 & 600 & 11.0 & \\
\hline & $11 / 4 / 93$ & 1230 & 7.8 & 601 & 11.5 & \\
\hline & $11 / 4 / 93$ & 1300 & 7.8 & 601 & 11.5 & QAS-32, replicate \\
\hline
\end{tabular}


Table 2.-Results of field measurements for $\mathrm{pH}$, specific conductance, and temperature of water, Naval Reactors Facility and vicinity-Continued

\begin{tabular}{|c|c|c|c|c|c|c|}
\hline Sample identifier & $\begin{array}{l}\text { Date sampled } \\
(\mathrm{m} / \mathrm{d} / \mathrm{y})\end{array}$ & Time & $\mathrm{pH}$ & $\begin{array}{c}\text { Specific } \\
\text { conductance }\end{array}$ & Temperature & Remarks \\
\hline \multirow[t]{11}{*}{ USGS 98} & $3 / 13 / 91$ & 0930 & 8.0 & 410 & 12.0 & \\
\hline & $6 / 7 / 91$ & 0900 & 8.0 & 405 & 12.5 & \\
\hline & $9 / 5 / 91$ & 1045 & 8.0 & 411 & 12.5 & \\
\hline & $12 / 3 / 91$ & 0940 & 8.0 & 395 & 12.5 & \\
\hline & $3 / 16 / 92$ & 1025 & 8.0 & 398 & 12.0 & \\
\hline & $6 / 16 / 92$ & 0940 & 8.0 & 400 & 12.0 & \\
\hline & $9 / 21 / 92$ & 1050 & 8.0 & 405 & 12.5 & \\
\hline & $9 / 21 / 92$ & 1030 & 8.0 & 405 & 12.5 & QAS-25, replicate \\
\hline & $12 / 8 / 92$ & 1310 & 8.1 & 393 & 12.0 & \\
\hline & $4 / 6 / 93$ & 1035 & 8.0 & 398 & 12.0 & \\
\hline & $6 / 8 / 93$ & 1510 & 8.0 & 398 & 12.0 & \\
\hline \multirow[t]{13}{*}{ USGS 99} & $3 / 13 / 91$ & 1340 & 8.0 & 515 & 11.5 & \\
\hline & $6 / 7 / 91$ & 0955 & 8.0 & 515 & 12.0 & \\
\hline & $9 / 5 / 91$ & 1450 & 8.0 & 525 & 12.0 & \\
\hline & $12 / 3 / 91$ & 1510 & 8.0 & 500 & 12.0 & \\
\hline & $3 / 16 / 92$ & 1455 & 8.0 & 501 & 12.0 & \\
\hline & $6 / 16 / 92$ & 1310 & 8.0 & 502 & 12.0 & \\
\hline & $6 / 16 / 92$ & 1345 & 8.0 & 502 & 12.0 & QAS-24, replicate \\
\hline & $9 / 21 / 92$ & 1205 & 7.9 & 510 & 12.0 & \\
\hline & $12 / 8 / 92$ & 1425 & 8.0 & 495 & 12.0 & \\
\hline & $4 / 6 / 93$ & 1140 & 7.9 & 498 & 11.5 & \\
\hline & $6 / 9 / 93$ & 1000 & 8.0 & 510 & 11.5 & \\
\hline & $9 / 13 / 93$ & 1230 & 7.8 & 520 & 11.0 & \\
\hline & $11 / 2 / 93$ & 1410 & 7.9 & 519 & 11.5 & \\
\hline \multirow[t]{4}{*}{ USGS 102} & $6 / 7 / 91$ & 1210 & 8.0 & 556 & 12.0 & \\
\hline & $9 / 5 / 91$ & 1250 & 8.0 & 575 & 12.0 & \\
\hline & $12 / 3 / 91$ & 1335 & 8.0 & 550 & 11.5 & \\
\hline & $3 / 16 / 92$ & 1300 & 8.0 & 555 & 12.0 & \\
\hline
\end{tabular}


Table 2.-Results of field measurements for $\mathrm{pH}$, specific conductance, and temperature of water, Naval Reactors Facility and vicinity-Continued

\begin{tabular}{|c|c|c|c|c|c|c|}
\hline Sample identifier & $\begin{array}{l}\text { Date sampled } \\
(\mathrm{m} / \mathrm{d} / \mathrm{y})\end{array}$ & Time & $\mathrm{pH}$ & $\begin{array}{c}\text { Specific } \\
\text { conductance }\end{array}$ & Temperature & Remarks \\
\hline \multirow[t]{8}{*}{ USGS 102 - cont. } & $6 / 16 / 92$ & 1455 & 8.0 & 550 & 12.0 & \\
\hline & $9 / 21 / 92$ & 1405 & 7.9 & 570 & 12.0 & \\
\hline & $12 / 9 / 92$ & 1405 & 8.0 & 557 & 12.0 & \\
\hline & $12 / 9 / 92$ & 1330 & 8.0 & 557 & 12.0 & QAS-27, replicate \\
\hline & $4 / 6 / 93$ & 1350 & 7.9 & 560 & 11.5 & \\
\hline & $6 / 9 / 93$ & 1245 & 8.0 & 570 & 12.5 & \\
\hline & $9 / 13 / 93$ & 1425 & 7.8 & 582 & 11.0 & \\
\hline & $11 / 4 / 93$ & 1340 & 7.8 & 587 & 11.5 & \\
\hline \multirow{13}{*}{$\begin{array}{l}\text { Water Supply } \\
\text { INEL-1 }\end{array}$} & $3 / 13 / 91$ & 1035 & 7.9 & 755 & 12.0 & \\
\hline & $6 / 5 / 91$ & 1040 & 8.0 & 730 & 12.0 & \\
\hline & $9 / 5 / 91$ & 0950 & 7.9 & 798 & 12.5 & \\
\hline & $12 / 3 / 91$ & 1100 & 7.9 & 725 & 12.0 & \\
\hline & $12 / 3 / 91$ & 1100 & 7.9 & 725 & 12.0 & QAS-19, replicate \\
\hline & $3 / 16 / 92$ & 0930 & 7.9 & 705 & 12.0 & \\
\hline & $6 / 16 / 92$ & 1125 & 7.9 & 718 & 12.0 & \\
\hline & $9 / 21 / 92$ & 0945 & 8.1 & 750 & 12.0 & \\
\hline & $12 / 8 / 92$ & 1155 & 8.0 & 708 & 12.0 & \\
\hline & $4 / 6 / 93$ & 0930 & 7.9 & 698 & 12.0 & \\
\hline & $6 / 8 / 93$ & 1345 & 7.9 & 710 & 12.5 & \\
\hline & $9 / 13 / 93$ & 1050 & 7.6 & 713 & 10.5 & \\
\hline & $11 / 2 / 93$ & 1230 & 7.8 & 689 & 12.0 & \\
\hline
\end{tabular}


Table 3.-Concentrations of dissolved anions and total recoverable sodium in water from round-three samples, Naval Reactors Facility and vicinity =

[Analyses were performed by the U.S. Geological Survey's National Water Quality Laboratory. Analytical results in milligrams per liter. Abbreviations: (m/d/y), month/day/year. Symbol: < indicates concentration is less than the specified laboratory reporting level. Sample identifier: see figure 2 for location of wells; QAS indicates quality-assurance sample, 23 and 30 are blank samples, others are replicates]

\begin{tabular}{|c|c|c|c|c|c|c|}
\hline $\begin{array}{l}\text { Sample } \\
\text { identifier }\end{array}$ & $\begin{array}{l}\text { Date sampled } \\
\qquad(\mathrm{m} / \mathrm{d} / \mathrm{y})\end{array}$ & Bromide & Chloride & Fluoride & Sulfate & Sodium \\
\hline QAS-23 & $6 / 12 / 92$ & 0.07 & 0.8 & $<0.1$ & $<0.1$ & 0.7 \\
\hline QAS-30 & $6 / 15 / 93$ & $<.01$ & $<.1$ & $<.1$ & .2 & $<.1$ \\
\hline \multirow[t]{3}{*}{ NRF-1 } & $3 / 5 / 91$ & .06 & 34 & .1 & 39 & 13 \\
\hline & $6 / 17 / 91$ & .07 & 37 & .2 & 38 & 14 \\
\hline & 9/9/91 & .07 & 31 & .2 & 36 & 15 \\
\hline QAS-18 & $9 / 9 / 91$ & .08 & 31 & .2 & 39 & 14 \\
\hline \multirow[t]{9}{*}{ NRF-1 } & $12 / 4 / 91$ & .08 & 36 & .2 & 42 & 12 \\
\hline & $3 / 11 / 92$ & .06 & 38 & .2 & 42 & 14 \\
\hline & $6 / 11 / 92$ & .08 & 40 & .2 & 41 & 15 \\
\hline & $9 / 16 / 92$ & .08 & 37 & .2 & 39 & 15 \\
\hline & $12 / 3 / 92$ & .08 & 36 & .2 & 41 & 15 \\
\hline & $4 / 7 / 93$ & .08 & 37 & .2 & 41 & 14 \\
\hline & $6 / 15 / 93$ & .08 & 39 & .2 & 43 & 13 \\
\hline & $9 / 15 / 93$ & .07 & 37 & .2 & 44 & 16 \\
\hline & $11 / 4 / 93$ & .07 & 33 & .2 & 40 & 16 \\
\hline NRF-2 & $3 / 5 / 91$ & .06 & 54 & .2 & 52 & 20 \\
\hline QAS-13 & $3 / 5 / 91$ & .07 & 55 & .1 & 53 & 19 \\
\hline \multirow[t]{7}{*}{ NRF-2 } & $6 / 17 / 91$ & .07 & 49 & .2 & 49 & 19 \\
\hline & 9/9/91 & .08 & 45 & .2 & 47 & 19 \\
\hline & $12 / 4 / 91$ & .08 & 52 & .2 & 55 & 12 \\
\hline & $3 / 11 / 92$ & .07 & 52 & .2 & 50 & 18 \\
\hline & $6 / 11 / 92$ & .08 & 54 & .2 & 50 & 20 \\
\hline & $9 / 16 / 92$ & .08 & 48 & .2 & 47 & 17 \\
\hline & $12 / 3 / 92$ & .07 & 43 & .1 & 47 & 19 \\
\hline
\end{tabular}


Table 3.-Concentrations of dissolved anions and total recoverable sodium in water from round-three samples, Naval Reactors Facility and vicinity-Continued

\begin{tabular}{|c|c|c|c|c|c|c|}
\hline $\begin{array}{l}\text { Sample } \\
\text { identifier }\end{array}$ & $\begin{array}{l}\text { Date sampled } \\
(\mathrm{m} / \mathrm{d} / \mathrm{y})\end{array}$ & Bromide & Chloride & Fluoride & Sulfate & Sodium \\
\hline \multirow[t]{3}{*}{ NRF-2 } & $4 / 7 / 93$ & .09 & 49 & .2 & 50 & 20 \\
\hline & $6 / 15 / 93$ & .09 & 45 & .2 & 49 & 14 \\
\hline & $9 / 15 / 93$ & .07 & 47 & .2 & 51 & 19 \\
\hline QAS-31 & $9 / 15 / 93$ & .07 & 48 & .2 & 53 & 19 \\
\hline NRF-2 & $11 / 4 / 93$ & .07 & 47 & .2 & 51 & 20 \\
\hline NRF-3 & $6 / 17 / 91$ & .07 & 36 & .2 & 38 & 13 \\
\hline QAS-16 & $6 / 17 / 91$ & .07 & 36 & .2 & 38 & 13 \\
\hline \multirow[t]{6}{*}{ NRF-3 } & 9/26/91 & .07 & 37 & .2 & 40 & 15 \\
\hline & $12 / 4 / 91$ & .07 & 36 & .2 & 42 & 10 \\
\hline & $3 / 11 / 92$ & .06 & 39 & .2 & 41 & 14 \\
\hline & $6 / 11 / 92$ & .07 & 41 & .2 & 40 & 14 \\
\hline & $9 / 16 / 92$ & .08 & 41 & .2 & 40 & 15 \\
\hline & $12 / 3 / 92$ & .08 & 35 & .1 & 41 & 14 \\
\hline QAS-26 & $12 / 3 / 92$ & .07 & 36 & .1 & 41 & 14 \\
\hline \multirow[t]{4}{*}{ NRF-3 } & $4 / 8 / 93$ & .09 & 37 & .2 & 40 & 15 \\
\hline & $6 / 15 / 93$ & .08 & 38 & .2 & 42 & 14 \\
\hline & $9 / 15 / 93$ & .07 & 38 & .2 & 43 & 16 \\
\hline & $11 / 4 / 93$ & .07 & 36 & .2 & 41 & 16 \\
\hline \multirow[t]{9}{*}{ NRF-4 } & $3 / 5 / 91$ & .07 & 42 & .1 & 45 & 17 \\
\hline & $6 / 17 / 91$ & .07 & 36 & .2 & 37 & 14 \\
\hline & 9/9/91 & .07 & 38 & .2 & 42 & 16 \\
\hline & $12 / 4 / 91$ & .08 & 46 & .2 & 51 & 9.3 \\
\hline & $3 / 11 / 92$ & .07 & 48 & .2 & 49 & 17 \\
\hline & $6 / 11 / 92$ & .07 & 45 & .2 & 44 & 16 \\
\hline & $9 / 16 / 92$ & .09 & 46 & .2 & 46 & 17 \\
\hline & $12 / 3 / 92$ & .08 & 35 & .2 & 36 & 14 \\
\hline & $4 / 7 / 93$ & .09 & 43 & .2 & 46 & 17 \\
\hline QAS-28 & $4 / 7 / 93$ & .09 & 45 & .2 & 46 & 18 \\
\hline
\end{tabular}


Table 3.-Concentrations of dissolved anions and total recoverable sodium in water from round-three samples, Naval Reactors Facility and vicinity-Continued

\begin{tabular}{|c|c|c|c|c|c|c|}
\hline $\begin{array}{l}\text { Sample } \\
\text { identifier }\end{array}$ & $\begin{array}{l}\text { Date sampled } \\
(\mathrm{m} / \mathrm{d} / \mathrm{y})\end{array}$ & Bromide & Chloride & Fluoride & Sulfate & Sodium \\
\hline \multirow[t]{3}{*}{ NRF-4 } & $6 / 15 / 93$ & .09 & 49 & .2 & 51 & 16 \\
\hline & $9 / 15 / 93$ & .08 & 49 & .2 & 54 & 19 \\
\hline & $11 / 4 / 93$ & .07 & 45 & .2 & 50 & 19 \\
\hline \multirow[t]{5}{*}{ NRF-6 } & $9 / 18 / 92$ & .09 & 210 & .3 & 240 & 90 \\
\hline & $12 / 9 / 92$ & .09 & 210 & .2 & 240 & 95 \\
\hline & $4 / 9 / 93$ & .10 & 230 & .2 & 250 & 100 \\
\hline & $9 / 14 / 93$ & .08 & 210 & .2 & 240 & 97 \\
\hline & $11 / 4 / 93$ & .08 & 200 & .2 & 230 & 95 \\
\hline \multirow[t]{6}{*}{ NRF-7 } & $9 / 18 / 92$ & .08 & 39 & .3 & 35 & 11 \\
\hline & $12 / 9 / 92$ & .03 & 5.5 & .2 & 16 & 8.4 \\
\hline & $4 / 9 / 93$ & .03 & 5.4 & .2 & 15 & 8.1 \\
\hline & $6 / 10 / 93$ & .03 & 5.5 & .2 & 15 & 7.8 \\
\hline & $9 / 14 / 93$ & .02 & 5 & .2 & 15 & 8.5 \\
\hline & $11 / 3 / 93$ & .01 & 4.8 & .3 & 15 & 8.7 \\
\hline \multirow[t]{2}{*}{ USGS 12} & $6 / 10 / 91$ & .08 & 31 & .2 & 26 & 13 \\
\hline & $9 / 6 / 91$ & .08 & 29 & .2 & 29 & 13 \\
\hline QAS-17 & $9 / 6 / 91$ & .08 & 30 & .2 & 32 & 14 \\
\hline \multirow[t]{9}{*}{ USGS 12} & $12 / 5 / 91$ & .08 & 36 & .2 & 36 & 12 \\
\hline & $3 / 12 / 92$ & .07 & 40 & .2 & 38 & 14 \\
\hline & $6 / 19 / 92$ & .08 & 40 & .2 & 35 & 15 \\
\hline & $9 / 18 / 92$ & .03 & 6.3 & .3 & 15 & 8.5 \\
\hline & $12 / 1 / 92$ & .01 & 7.1 & .2 & 19 & 7.7 \\
\hline & $4 / 13 / 93$ & .1 & 37 & .2 & 36 & 14 \\
\hline & $6 / 14 / 93$ & .09 & 38 & .2 & 37 & 13 \\
\hline & $9 / 16 / 93$ & .08 & 36 & .2 & 38 & 15 \\
\hline & $11 / 5 / 93$ & .08 & 37 & .2 & 35 & 16 \\
\hline QAS-33 & $11 / 5 / 93$ & .08 & 33 & .2 & 36 & 16 \\
\hline USGS 15 & $6 / 10 / 91$ & .04 & 11 & .2 & 15 & 12 \\
\hline
\end{tabular}


Table 3.-Concentrations of dissolved anions and total recoverable sodium in water from round-three samples, Naval Reactors Facility and vicinity-Continued

\begin{tabular}{|c|c|c|c|c|c|c|}
\hline $\begin{array}{l}\text { Sample } \\
\text { identifier }\end{array}$ & $\begin{array}{l}\text { Date sampled } \\
\quad(\mathrm{m} / \mathrm{d} / \mathrm{y})\end{array}$ & Bromide & Chloride & Fluoride & Sulfate & Sodium \\
\hline \multirow[t]{3}{*}{ USGS 15} & $9 / 6 / 91$ & .05 & 19 & .1 & 24 & 17 \\
\hline & $12 / 4 / 91$ & .03 & 10 & .2 & 22 & 6.1 \\
\hline & $3 / 12 / 92$ & .02 & 8.1 & .1 & 18 & 5.7 \\
\hline QAS-22 & $3 / 12 / 92$ & .01 & 8.1 & .2 & 19 & 8.7 \\
\hline \multirow[t]{7}{*}{ USGS 15} & $6 / 19 / 92$ & .04 & 21 & .2 & 25 & 17 \\
\hline & $9 / 17 / 92$ & .05 & 18 & .2 & 24 & 14 \\
\hline & $12 / 1 / 92$ & .08 & 43 & .2 & 47 & 18 \\
\hline & $4 / 13 / 93$ & .04 & 6.5 & .1 & 18 & 7.2 \\
\hline & $6 / 14 / 93$ & .03 & 9.3 & .1 & 20 & 8.6 \\
\hline & $9 / 16 / 93$ & .04 & 14 & $<.1$ & 25 & 14 \\
\hline & $11 / 5 / 93$ & .02 & 9 & .2 & 19 & 11 \\
\hline USGS 17 & $3 / 13 / 91$ & .02 & 7.1 & .2 & 19 & 5.6 \\
\hline QAS-14 & $3 / 13 / 91$ & .02 & 6.9 & .2 & 18 & 5.4 \\
\hline \multirow[t]{9}{*}{ USGS 17} & $6 / 6 / 91$ & .02 & 7.4 & .2 & 12 & 6.5 \\
\hline & 9/5/91 & .03 & 7.2 & .3 & 22 & 6.3 \\
\hline & $12 / 3 / 91$ & .03 & 7.1 & .3 & 22 & 5.7 \\
\hline & $3 / 16 / 92$ & .02 & 7.0 & .3 & 19 & 5.4 \\
\hline & $6 / 11 / 92$ & .02 & 7.1 & .2 & 22 & 6.7 \\
\hline & $9 / 16 / 92$ & .03 & 7.0 & .3 & 19 & 7.0 \\
\hline & $12 / 3 / 92$ & .02 & 5.9 & .2 & 20 & 6.4 \\
\hline & $4 / 7 / 93$ & .04 & 10 & .2 & 19 & 6.8 \\
\hline & $6 / 11 / 93$ & .03 & 5.9 & .2 & 19 & 5.6 \\
\hline QAS-29 & $6 / 11 / 93$ & .03 & 5.9 & .2 & 19 & 5.8 \\
\hline \multirow[t]{2}{*}{ USGS 17} & $9 / 15 / 93$ & .02 & 6.0 & .2 & 19 & 6.7 \\
\hline & $11 / 8 / 93$ & .01 & 5.6 & .2 & 19 & 6.9 \\
\hline \multirow[t]{2}{*}{ USGS 97} & $3 / 13 / 91$ & .07 & 34 & .2 & 36 & 12 \\
\hline & $6 / 7 / 91$ & .08 & 29 & .2 & 27 & 13 \\
\hline QAS-15 & $6 / 7 / 91$ & .08 & 28 & .2 & 33 & 15 \\
\hline
\end{tabular}


Table 3.-Concentrations of dissolved anions and total recoverable sodium in water from round-three samples, Naval Reactors Facility and vicinity-Continued

\begin{tabular}{|c|c|c|c|c|c|c|}
\hline $\begin{array}{l}\text { Sample } \\
\text { identifier }\end{array}$ & $\begin{array}{l}\text { Date sampled } \\
\qquad(\mathrm{m} / \mathrm{d} / \mathrm{y})\end{array}$ & Bromide & Chloride & Fluoride & Sulfate & Sodium \\
\hline \multirow[t]{10}{*}{ USGS 97} & $9 / 5 / 91$ & .08 & 37 & .2 & 38 & 14 \\
\hline & $12 / 3 / 91$ & .08 & 36 & .2 & 38 & 12 \\
\hline & $3 / 16 / 92$ & .07 & 33 & .2 & 34 & 11 \\
\hline & $6 / 17 / 92$ & .08 & 32 & .2 & 36 & 14 \\
\hline & $9 / 21 / 92$ & .09 & 38 & .2 & 36 & 15 \\
\hline & $12 / 8 / 92$ & .08 & 33 & .1 & 37 & 13 \\
\hline & $4 / 6 / 93$ & .09 & 34 & .2 & 36 & 15 \\
\hline & $6 / 9 / 93$ & .09 & 34 & .1 & 37 & 12 \\
\hline & $9 / 13 / 93$ & .08 & 35 & .2 & 37 & 14 \\
\hline & $11 / 4 / 93$ & .07 & 32 & .2 & 36 & 15 \\
\hline QAS-32 & $11 / 4 / 93$ & .08 & 32 & .2 & 36 & 15 \\
\hline \multirow[t]{7}{*}{ USGS 98} & $3 / 13 / 91$ & .04 & 15 & .2 & 22 & 8.7 \\
\hline & $6 / 7 / 91$ & .04 & 16 & $<.1$ & 11 & 10 \\
\hline & $9 / 5 / 91$ & .05 & 14 & .2 & 21 & 8.8 \\
\hline & $12 / 3 / 91$ & .04 & 17 & .2 & 24 & 7.8 \\
\hline & $3 / 16 / 92$ & .03 & 17 & .2 & 21 & 8.3 \\
\hline & $6 / 16 / 92$ & .04 & 14 & .2 & 33 & 9.3 \\
\hline & $9 / 21 / 92$ & .05 & 17 & .2 & 23 & 8.6 \\
\hline QAS-25 & $9 / 21 / 92$ & .05 & 18 & .3 & 21 & 11 \\
\hline \multirow[t]{3}{*}{ USGS 98} & $12 / 8 / 92$ & .05 & 14 & .2 & 22 & 9.5 \\
\hline & $4 / 6 / 93$ & .05 & 14 & .2 & 21 & 9.7 \\
\hline & $6 / 8 / 93$ & .04 & 18 & .2 & 22 & 8.9 \\
\hline \multirow[t]{6}{*}{ USGS 99} & $3 / 13 / 91$ & .06 & 21 & .1 & 26 & 10 \\
\hline & $6 / 7 / 91$ & .05 & 17 & .2 & 18 & 12 \\
\hline & $9 / 5 / 91$ & .06 & 24 & .2 & 30 & 12 \\
\hline & $12 / 3 / 91$ & .05 & 20 & .2 & 27 & 8.8 \\
\hline & $3 / 16 / 92$ & .05 & 22 & .2 & 25 & 11 \\
\hline & $6 / 16 / 92$ & .05 & 20 & .2 & 27 & 12 \\
\hline
\end{tabular}


Table 3.-Concentrations of dissolved anions and total recoverable sodium in water from round-three samples, Naval Reactors Facility and vicinity-Continued

\begin{tabular}{|c|c|c|c|c|c|c|}
\hline $\begin{array}{c}\text { Sample } \\
\text { identifier }\end{array}$ & $\begin{array}{l}\text { Date sampled } \\
\qquad(\mathrm{m} / \mathrm{d} / \mathrm{y})\end{array}$ & Bromide & Chloride & Fluoride & Sulfate & Sodium \\
\hline QAS-24 & $6 / 16 / 92$ & .05 & 20 & .2 & 26 & 12 \\
\hline \multirow[t]{6}{*}{ USGS 99} & $9 / 21 / 92$ & .06 & 23 & .1 & 28 & 13 \\
\hline & $12 / 8 / 92$ & .06 & 18 & .1 & 27 & 12 \\
\hline & $4 / 6 / 93$ & .07 & 20 & .1 & 26 & 12 \\
\hline & $6 / 9 / 93$ & .05 & 20 & .1 & 27 & 11 \\
\hline & $9 / 13 / 93$ & .05 & 19 & .2 & 27 & 13 \\
\hline & $11 / 2 / 93$ & .04 & 18 & .2 & 27 & 14 \\
\hline \multirow[t]{7}{*}{ USGS 102} & $6 / 7 / 91$ & .07 & 23 & .2 & 23 & 12 \\
\hline & $9 / 5 / 91$ & .07 & 36 & .2 & 39 & 13 \\
\hline & $12 / 3 / 91$ & .07 & 32 & .2 & 35 & 9.7 \\
\hline & $3 / 16 / 92$ & .07 & 31 & .2 & 34 & 11 \\
\hline & $6 / 16 / 92$ & .12 & 30 & .2 & 35 & 13 \\
\hline & $9 / 21 / 92$ & .08 & 35 & .2 & 35 & 13 \\
\hline & $12 / 9 / 92$ & .07 & 31 & .2 & 36 & 13 \\
\hline QAS-27 & $12 / 9 / 92$ & .07 & 31 & .2 & 36 & 13 \\
\hline \multirow[t]{4}{*}{ USGS 102} & $4 / 6 / 93$ & .09 & 31 & .2 & 35 & 12 \\
\hline & 6/9/93 & .09 & 33 & .2 & 36 & 12 \\
\hline & $9 / 13 / 93$ & .08 & 33 & .2 & 36 & 14 \\
\hline & $11 / 4 / 93$ & .09 & 30 & .2 & 35 & 14 \\
\hline \multirow{4}{*}{$\begin{array}{l}\text { Water Supply } \\
\text { INEL-1 }\end{array}$} & $3 / 13 / 91$ & .30 & 100 & .1 & 50 & 15 \\
\hline & $6 / 5 / 91$ & .31 & 96 & $<.1$ & 46 & 18 \\
\hline & 9/5/91 & .33 & 99 & .2 & 53 & 19 \\
\hline & $12 / 3 / 91$ & .31 & 110 & .2 & 61 & 15 \\
\hline QAS-19 & $12 / 3 / 91$ & .31 & 100 & .2 & 55 & 15 \\
\hline \multirow{3}{*}{$\begin{array}{l}\text { Water Supply } \\
\text { INEL-1 }\end{array}$} & $3 / 16 / 92$ & .28 & 94 & $<.1$ & 49 & 14 \\
\hline & $6 / 16 / 92$ & .30 & 99 & .2 & 53 & 18 \\
\hline & $9 / 21 / 92$ & .32 & 80 & .2 & 40 & 13 \\
\hline
\end{tabular}


Table 3.-Concentrations of dissolved anions and total recoverable sodium in water from round-three samples, Naval Reactors Facility and vicinity-Continued

\begin{tabular}{lcccccc}
\hline $\begin{array}{c}\text { Sample } \\
\text { identifier }\end{array}$ & $\begin{array}{c}\text { Date sampled } \\
(\mathrm{m} / \mathrm{d} / \mathrm{y})\end{array}$ & Bromide & Chloride & Fluoride & Sulfate & Sodium \\
\hline $\begin{array}{l}\text { Water Supply } \\
\text { INEL-1 }\end{array}$ & $12 / 8 / 92$ & .30 & 96 & .1 & 53 & 18 \\
& $4 / 6 / 93$ & .29 & 94 & .1 & 50 & 18 \\
& $6 / 8 / 93$ & .28 & 94 & .1 & 52 & 16 \\
& $9 / 13 / 93$ & .27 & 89 & .2 & 50 & 18 \\
& $11 / 2 / 93$ & .24 & 80 & .2 & 49 & 18 \\
\hline
\end{tabular}


Table 4.-Statistical parameters for dissolved anions and total recoverable sodium, by well =

[See figure 2 for location of wells. Units are milligrams per liter. Symbol: < indicates concentration is less than the specified laboratory reporting level. Values are derived from tables 3,12 , and 18. Quality-assurance replicates are included in the calculation of statistical parameters. Mean and median sample size: includes all samples with concentrations greater than laboratory reporting level]

\begin{tabular}{|c|c|c|c|c|c|c|}
\hline \multirow{2}{*}{ Constituent } & \multicolumn{6}{|c|}{ Statistical parameter } \\
\hline & Minimum & Maximum & Median & Mean & Sample size & $\begin{array}{l}\text { Mean and median } \\
\text { sample size }\end{array}$ \\
\hline & \multicolumn{6}{|c|}{ NRF-1 } \\
\hline Bromide & 0.06 & 0.08 & 0.08 & 0.07 & 13 & 13 \\
\hline Chloride & 31 & 40 & 37 & 36 & 13 & 13 \\
\hline Fluoride & .1 & .2 & .2 & .2 & 13 & 13 \\
\hline Sulfate & 36 & 44 & 41 & 40 & 13 & 13 \\
\hline \multirow[t]{2}{*}{ Sodium } & 12 & 16 & 14 & 14 & 13 & 13 \\
\hline & \multicolumn{6}{|c|}{ NRF-2 } \\
\hline Bromide & .06 & .09 & .07 & .08 & 14 & 14 \\
\hline Chloride & 43 & 55 & 48.5 & 49 & 14 & 14 \\
\hline Fluoride & .1 & .2 & .2 & .2 & 14 & 14 \\
\hline Sulfate & 47 & 55 & 50 & 50 & 14 & 14 \\
\hline \multirow[t]{2}{*}{ Sodium } & 12 & 20 & 19 & 18 & 14 & 14 \\
\hline & \multicolumn{6}{|c|}{ NRF-3 } \\
\hline Bromide & .06 & .09 & .07 & .07 & 13 & 13 \\
\hline Chloride & 35 & 41 & 37 & 37 & 13 & 13 \\
\hline Fluoride & .1 & .2 & .2 & .2 & 13 & 13 \\
\hline Sulfate & 38 & 43 & 41 & 41 & 13 & 13 \\
\hline \multirow[t]{2}{*}{ Sodium } & 10 & 16 & 14 & 14 & 13 & 13 \\
\hline & \multicolumn{6}{|c|}{ NRF-4 } \\
\hline Bromide & .07 & .09 & .08 & .08 & 13 & 13 \\
\hline Chloride & 35 & 49 & 45 & 44 & 13 & 13 \\
\hline Fluoride & .1 & .2 & .2 & .2 & 13 & 13 \\
\hline Sulfate & 36 & 54 & 46 & 46 & 13 & 13 \\
\hline Sodium & 9.3 & 19 & 17 & 16 & 13 & 13 \\
\hline
\end{tabular}


Table 4.-Statistical parameters for dissolved anions and total recoverable sodium, by well-Continued

\begin{tabular}{|c|c|c|c|c|c|c|}
\hline \multirow{2}{*}{ Constituent } & \multicolumn{6}{|c|}{ Statistical parameter } \\
\hline & Minimum & Maximum & Median & Mean & Sample size & $\begin{array}{l}\text { Mean and median } \\
\text { sample size }\end{array}$ \\
\hline & \multicolumn{6}{|c|}{ NRF-6 } \\
\hline Bromide & .08 & .10 & .08 & .09 & 12 & 12 \\
\hline Chloride & 190 & 230 & 205 & 206 & 12 & 12 \\
\hline Fluoride & $<.1$ & .3 & .2 & .2 & 12 & 10 \\
\hline Sulfate & 210 & 250 & 235 & 232 & 12 & 12 \\
\hline \multirow[t]{2}{*}{ Sodium } & 54 & 100 & 90 & 86 & 12 & 12 \\
\hline & \multicolumn{6}{|c|}{ NRF-7 } \\
\hline Bromide & .01 & .08 & .02 & .03 & 13 & 13 \\
\hline Chloride & 4.8 & 39 & 5.5 & 8.3 & 13 & 13 \\
\hline Fluoride & .2 & .5 & .2 & .3 & 13 & 13 \\
\hline Sulfate & 14 & 35 & 16 & 18 & 13 & 13 \\
\hline \multirow[t]{2}{*}{ Sodium } & 7.0 & 11 & 8.5 & 8.6 & 13 & 13 \\
\hline & \multicolumn{6}{|c|}{ USGS 12} \\
\hline Bromide & .01 & .1 & .08 & .07 & 13 & 13 \\
\hline Chloride & 6.3 & 40 & 36 & 31 & 13 & 13 \\
\hline Fluoride & .2 & .3 & .2 & .2 & 13 & 13 \\
\hline Sulfate & 15 & 38 & 35 & 32 & 13 & 13 \\
\hline \multirow[t]{2}{*}{ Sodium } & 7.7 & 16 & 14 & 13 & 13 & 13 \\
\hline & \multicolumn{6}{|c|}{ USGS 15} \\
\hline Bromide & .01 & .08 & .04 & .04 & 12 & 12 \\
\hline Chloride & 6.5 & 43 & 10.5 & 15 & 12 & 12 \\
\hline Fluoride & $<.1$ & .2 & .2 & .2 & 12 & 11 \\
\hline Sulfate & 15 & 47 & 21 & 23 & 12 & 12 \\
\hline Sodium & 5.7 & 18 & 11.5 & 12 & 12 & 12 \\
\hline
\end{tabular}


Table 4.-Statistical parameters for dissolved anions and total recoverable sodium, by well-Continued

\begin{tabular}{|c|c|c|c|c|c|c|}
\hline \multirow{2}{*}{ Constituent } & \multicolumn{6}{|c|}{ Statistical parameter } \\
\hline & Minimum & Maximum & Median & Mean & Sample size & $\begin{array}{l}\text { Mean and median } \\
\text { sample size }\end{array}$ \\
\hline & \multicolumn{6}{|c|}{ USGS 17} \\
\hline Bromide & .01 & .04 & .02 & .02 & 14 & 14 \\
\hline Chloride & 5.6 & 10 & 7.0 & 6.9 & 14 & 14 \\
\hline Fluoride & .2 & .3 & .2 & .2 & 14 & 14 \\
\hline Sulfate & 12 & 22 & 19 & 19 & 14 & 14 \\
\hline \multirow[t]{2}{*}{ Sodium } & 5.4 & 7.0 & 6.35 & 6.2 & 14 & 14 \\
\hline & \multicolumn{6}{|c|}{ USGS 97} \\
\hline Bromide & .07 & .09 & .08 & .08 & 14 & 14 \\
\hline Chloride & 28 & 38 & 33.5 & 33 & 14 & 14 \\
\hline Fluoride & .1 & .2 & .2 & .2 & 14 & 14 \\
\hline Sulfate & 27 & 38 & 36 & 36 & 14 & 14 \\
\hline \multirow[t]{2}{*}{ Sodium } & 11 & 15 & 14 & 14 & 14 & 14 \\
\hline & \multicolumn{6}{|c|}{ USGS 98} \\
\hline Bromide & .03 & .05 & .04 & .04 & 11 & 11 \\
\hline Chloride & 14 & 18 & 16 & 16 & 11 & 11 \\
\hline Fluoride & $<.1$ & .3 & .2 & .2 & 11 & 10 \\
\hline Sulfate & 11 & 33 & 22 & 22 & 11 & 11 \\
\hline \multirow[t]{2}{*}{ Sodium } & 7.8 & 11 & 8.9 & 9.1 & 11 & 11 \\
\hline & \multicolumn{6}{|c|}{ USGS 99} \\
\hline Bromide & .04 & .07 & .05 & .05 & 13 & 13 \\
\hline Chloride & 17 & 24 & 20 & 20 & 13 & 13 \\
\hline Fluoride & .1 & .2 & .2 & .2 & 13 & 13 \\
\hline Sulfate & 18 & 30 & 27 & 26 & 13 & 13 \\
\hline Sodium & 8.8 & 14 & 12 & 12 & 13 & 13 \\
\hline
\end{tabular}


Table 4.-Statistical parameters for dissolved anions and total recoverable sodium, by well-Continued

\begin{tabular}{|c|c|c|c|c|c|c|}
\hline \multirow{2}{*}{ Constituent } & \multicolumn{6}{|c|}{ Statistical parameter } \\
\hline & Minimum & Maximum & Median & Mean & Sample size & $\begin{array}{l}\text { Mean and median } \\
\text { sample size }\end{array}$ \\
\hline & \multicolumn{6}{|c|}{ USGS 102} \\
\hline Bromide & .07 & .12 & .075 & .08 & 12 & 12 \\
\hline Chloride & 23 & 36 & 31 & 31 & 12 & 12 \\
\hline Fluoride & .2 & .2 & .2 & .2 & 12 & 12 \\
\hline Sulfate & 23 & 39 & 35 & 35 & 12 & 12 \\
\hline \multirow[t]{2}{*}{ Sodium } & 9.7 & 14 & 13 & 12 & 12 & 12 \\
\hline & \multicolumn{6}{|c|}{ Water Supply INEL-1 } \\
\hline Bromide & .24 & .33 & .30 & .30 & 13 & 13 \\
\hline Chloride & 80 & 110 & 96 & 95 & 13 & 13 \\
\hline Fluoride & $<.1$ & .2 & .2 & .2 & 13 & 11 \\
\hline Sulfate & 40 & 61 & 50 & 51 & 13 & 13 \\
\hline Sodium & 13 & 19 & 18 & 17 & 13 & 13 \\
\hline
\end{tabular}


Table 5.-Concentrations of selected total recoverable trace elements in water from roundthree samples, Naval Reactors Facility and vicinity

[Analyses were performed by the U.S. Geological Survey's National Water Quality Laboratory. Analytical results in micrograms per liter. Abbreviations: (m/d/y), month/day/year. Symbols: < indicates concentration is less than the specified laboratory reporting level; SR indicates sample ruined. Sample identifier: see figure 2 for location of wells; QAS indicates quality-assurance sample, 23 and 30 are blank samples, others are replicates]

\begin{tabular}{|c|c|c|c|c|c|c|c|}
\hline $\begin{array}{l}\text { Sample } \\
\text { identifier }\end{array}$ & $\begin{array}{l}\text { Date sampled } \\
\qquad(\mathrm{m} / \mathrm{d} / \mathrm{y})\end{array}$ & Chromium & Iron & Lead & Mercury & Nickel & Silver \\
\hline QAS-23 & $6 / 12 / 92$ & $<1$ & $<10$ & $<1$ & $<0.1$ & $<1$ & $<1$ \\
\hline QAS-30 & $6 / 15 / 93$ & $<1$ & 110 & 17 & $<.1$ & $<1$ & $<1$ \\
\hline \multirow[t]{3}{*}{ NRF-1 } & $3 / 5 / 91$ & 9 & 190 & 1 & $<.1$ & 2 & $<1$ \\
\hline & $6 / 17 / 91$ & 9 & 140 & 4 & $<.1$ & 6 & $<1$ \\
\hline & 9/9/91 & 12 & 470 & 1 & $<.1$ & 2 & $<1$ \\
\hline QAS-18 & 9/9/91 & 11 & 240 & $<1$ & $<.1$ & 2 & $<1$ \\
\hline \multirow[t]{9}{*}{ NRF-1 } & $12 / 4 / 91$ & 8 & 190 & 2 & $<.1$ & 1 & $<1$ \\
\hline & $3 / 11 / 92$ & 9 & 890 & 2 & $<.1$ & 2 & $<1$ \\
\hline & $6 / 11 / 92$ & 6 & 120 & 3 & $<.1$ & $<1$ & $<1$ \\
\hline & $9 / 16 / 92$ & 7 & 80 & $<1$ & $<.1$ & 1 & $<1$ \\
\hline & $12 / 3 / 92$ & 13 & 620 & $<1$ & $<.1$ & $<1$ & 4 \\
\hline & $4 / 7 / 93$ & 21 & 3,200 & 3 & $<.1$ & $<1$ & $<1$ \\
\hline & $6 / 15 / 93$ & 9 & 540 & $<1$ & $<.1$ & $<1$ & $<1$ \\
\hline & $9 / 15 / 93$ & 6 & 30 & $<1$ & $<.1$ & $<1$ & $<1$ \\
\hline & $11 / 4 / 93$ & 8 & 150 & $<1$ & $<.1$ & $\mathrm{SR}$ & $<1$ \\
\hline NRF-2 & $3 / 5 / 91$ & 14 & $<10$ & $<1$ & $<.1$ & $<1$ & $<1$ \\
\hline QAS-13 & $3 / 5 / 91$ & 15 & 10 & $<1$ & $<.1$ & $<1$ & $<1$ \\
\hline \multirow[t]{9}{*}{ NRF-2 } & $6 / 17 / 91$ & 13 & 20 & 1 & $<.1$ & 5 & $<1$ \\
\hline & 9/9/91 & 11 & 30 & 4 & $<.1$ & 2 & $<1$ \\
\hline & $12 / 4 / 91$ & 13 & $<10$ & $<1$ & $<.1$ & 2 & $<1$ \\
\hline & $3 / 11 / 92$ & $<1$ & $<10$ & $<1$ & $<.1$ & $<1$ & $<1$ \\
\hline & $6 / 11 / 92$ & 11 & 20 & $<1$ & $<.1$ & $<1$ & $<1$ \\
\hline & $9 / 16 / 92$ & 10 & 80 & $<1$ & $<.1$ & $<1$ & $<1$ \\
\hline & $12 / 3 / 92$ & 12 & 30 & $<1$ & $<.1$ & 2 & $<1$ \\
\hline & $4 / 7 / 93$ & 12 & $<10$ & $<1$ & $<.1$ & $<1$ & $<1$ \\
\hline & $6 / 15 / 93$ & 6 & 10 & $<1$ & $<.1$ & $<1$ & $<1$ \\
\hline
\end{tabular}


Table 5.-Concentrations of selected total recoverable trace elements in water from roundthree samples, Naval Reactors Facility and vicinity-Continued

\begin{tabular}{|c|c|c|c|c|c|c|c|}
\hline $\begin{array}{l}\text { Sample } \\
\text { identifier }\end{array}$ & $\begin{array}{l}\text { Date sampled } \\
\qquad(\mathrm{m} / \mathrm{d} / \mathrm{y})\end{array}$ & Chromium & Iron & Lead & Mercury & Nickel & Silver \\
\hline NRF-2 & $9 / 15 / 93$ & 9 & 20 & $<1$ & $<.1$ & $<1$ & $<1$ \\
\hline QAS-31 & $9 / 15 / 93$ & 10 & $<10$ & $<1$ & $<.1$ & $<1$ & $<1$ \\
\hline NRF-2 & $11 / 4 / 93$ & 9 & 20 & $<1$ & $<.1$ & SR & $<1$ \\
\hline NRF-3 & $6 / 17 / 91$ & 7 & 60 & 2 & $<.1$ & 4 & $<1$ \\
\hline QAS-16 & $6 / 17 / 91$ & 7 & 120 & 2 & $<.1$ & 3 & $<1$ \\
\hline \multirow[t]{6}{*}{ NRF-3 } & $9 / 26 / 91$ & 9 & 300 & 2 & $<.1$ & 1 & $<1$ \\
\hline & $12 / 4 / 91$ & 6 & 120 & $<1$ & $<.1$ & 2 & $<1$ \\
\hline & $3 / 11 / 92$ & 8 & 920 & $<1$ & $<.1$ & $<1$ & $<1$ \\
\hline & $6 / 11 / 92$ & 4 & 30 & $<1$ & $<.1$ & $<1$ & $<1$ \\
\hline & $9 / 16 / 92$ & 13 & 4,900 & 3 & $<.1$ & 14 & $<1$ \\
\hline & $12 / 3 / 92$ & 10 & 320 & $<1$ & $<.1$ & 2 & $<1$ \\
\hline QAS-26 & $12 / 3 / 92$ & 4 & 100 & $<1$ & $<.1$ & $<1$ & $<1$ \\
\hline \multirow[t]{4}{*}{ NRF-3 } & $4 / 8 / 93$ & 7 & 140 & 1 & $<.1$ & $<1$ & $<1$ \\
\hline & $6 / 15 / 93$ & 6 & 170 & $<1$ & $<.1$ & $<1$ & $<1$ \\
\hline & $9 / 15 / 93$ & 5 & 630 & 2 & $<.1$ & $<1$ & $<1$ \\
\hline & $11 / 4 / 93$ & 6 & 90 & $<1$ & $<.1$ & SR & $<1$ \\
\hline \multirow[t]{9}{*}{ NRF-4 } & $3 / 5 / 91$ & 8 & 70 & 1 & $<.1$ & $<1$ & $<1$ \\
\hline & $6 / 17 / 91$ & 12 & 40 & 1 & $<.1$ & $<1$ & $<1$ \\
\hline & 9/9/91 & 10 & 170 & $<1$ & $<.1$ & 2 & $<1$ \\
\hline & $12 / 4 / 91$ & 10 & 20 & $<1$ & $<.1$ & 2 & $<1$ \\
\hline & $3 / 11 / 92$ & 11 & $<10$ & $<1$ & $<.1$ & $<1$ & $<1$ \\
\hline & $6 / 11 / 92$ & 6 & 20 & $<1$ & $<.1$ & $<1$ & $<1$ \\
\hline & $9 / 16 / 92$ & 10 & 300 & $<1$ & $<.1$ & $<1$ & $<1$ \\
\hline & $12 / 3 / 92$ & 6 & 100 & $<1$ & $<.1$ & $<1$ & $<1$ \\
\hline & $4 / 7 / 93$ & 9 & $<10$ & $<1$ & $<.1$ & $<1$ & $<1$ \\
\hline QAS-28 & $4 / 7 / 93$ & 9 & $<10$ & $<1$ & $<.1$ & $<1$ & $<1$ \\
\hline \multirow[t]{3}{*}{ NRF-4 } & $6 / 15 / 93$ & 8 & $<10$ & $<1$ & $<.1$ & $<1$ & $<1$ \\
\hline & $9 / 15 / 93$ & 10 & $<10$ & $<1$ & $<.1$ & $<1$ & $<1$ \\
\hline & $11 / 4 / 93$ & 9 & 10 & $<1$ & $<.1$ & $\mathrm{SR}$ & $<1$ \\
\hline NRF-6 & $9 / 18 / 92$ & $<1$ & 140 & $<1$ & $<.1$ & 10 & $<1$ \\
\hline
\end{tabular}


Table 5.-Concentrations of selected total recoverable trace elements in water from roundthree samples, Naval Reactors Facility and vicinity-Continued

\begin{tabular}{|c|c|c|c|c|c|c|c|}
\hline $\begin{array}{l}\text { Sample } \\
\text { identifier }\end{array}$ & $\begin{array}{l}\text { Date sampled } \\
\qquad(\mathrm{m} / \mathrm{d} / \mathrm{y})\end{array}$ & Chromium & Iron & Lead & Mercury & Nickel & Silver \\
\hline \multirow[t]{4}{*}{ NRF-6 } & $12 / 9 / 92$ & 41 & 100 & $<1$ & $<.1$ & 8 & $<1$ \\
\hline & 4/9/93 & 11 & 280 & $<1$ & $<.1$ & 13 & $<1$ \\
\hline & $9 / 14 / 93$ & 42 & 1,100 & $<1$ & $<.1$ & 48 & $<1$ \\
\hline & $11 / 4 / 93$ & 32 & 400 & $<1$ & $<.1$ & SR & $<1$ \\
\hline \multirow[t]{6}{*}{ NRF-7 } & $9 / 18 / 92$ & 5 & 180 & $<1$ & $<.1$ & $<1$ & $<1$ \\
\hline & $12 / 9 / 92$ & 11 & 210 & $<1$ & $<.1$ & 6 & $<1$ \\
\hline & 4/9/93 & 13 & 330 & $<1$ & $<.1$ & 5 & $<1$ \\
\hline & $6 / 10 / 93$ & 11 & 600 & $<1$ & $<.1$ & 6 & $<1$ \\
\hline & $9 / 14 / 93$ & 10 & 180 & $<1$ & $<.1$ & 4 & $<1$ \\
\hline & $11 / 3 / 93$ & 12 & 330 & $<1$ & $<.1$ & $\mathrm{SR}$ & $<1$ \\
\hline \multirow[t]{2}{*}{ USGS 12} & $6 / 10 / 91$ & 4 & 120 & 1 & $<.1$ & 1 & $<1$ \\
\hline & $9 / 6 / 91$ & 7 & 50 & $<1$ & $<.1$ & 1 & $<1$ \\
\hline QAS-17 & $9 / 6 / 91$ & 6 & 90 & $<1$ & $<.1$ & 2 & $<1$ \\
\hline \multirow[t]{9}{*}{ USGS 12} & $12 / 5 / 91$ & 6 & 70 & $<1$ & $<.1$ & 1 & $<1$ \\
\hline & $3 / 12 / 92$ & 7 & 20 & $<1$ & $<.1$ & $<1$ & $<1$ \\
\hline & $6 / 19 / 92$ & 5 & 50 & $<1$ & $<.1$ & 1 & $<1$ \\
\hline & $9 / 18 / 92$ & 9 & 260 & $<1$ & $<.1$ & 6 & $<1$ \\
\hline & $12 / 1 / 92$ & 10 & 3,000 & $<1$ & $<.1$ & 7 & $<1$ \\
\hline & $4 / 13 / 93$ & 5 & 40 & $<1$ & $<.1$ & $<1$ & $<1$ \\
\hline & $6 / 14 / 93$ & 5 & 40 & $<1$ & $<.1$ & 1 & $<1$ \\
\hline & $9 / 16 / 93$ & 6 & 40 & $<1$ & $<.1$ & $<1$ & $<1$ \\
\hline & $11 / 5 / 93$ & 5 & 140 & $<1$ & $<.1$ & 2 & $<1$ \\
\hline QAS-33 & $11 / 5 / 93$ & 6 & 270 & $<1$ & $<.1$ & 2 & $<1$ \\
\hline \multirow[t]{4}{*}{ USGS 15} & $6 / 10 / 91$ & 3 & 30 & 2 & $<.1$ & 1 & $<1$ \\
\hline & $9 / 6 / 91$ & 6 & 40 & $<1$ & $<.1$ & 2 & $<1$ \\
\hline & $12 / 4 / 91$ & 8 & $<10$ & $<1$ & $<.1$ & 1 & $<1$ \\
\hline & $3 / 12 / 92$ & 6 & 10 & $<1$ & $<.1$ & $<1$ & $<1$ \\
\hline QAS-22 & $3 / 12 / 92$ & 8 & $<10$ & $<1$ & $<.1$ & $<1$ & $<1$ \\
\hline \multirow[t]{2}{*}{ USGS 15} & $6 / 19 / 92$ & 71 & 30,000 & 16 & $<.1$ & 86 & $<1$ \\
\hline & $9 / 17 / 92$ & $<1$ & 250 & $<1$ & $<.1$ & 1 & $<1$ \\
\hline
\end{tabular}


Table 5.-Concentrations of selected total recoverable trace elements in water from roundthree samples, Naval Reactors Facility and vicinity-Continued

\begin{tabular}{|c|c|c|c|c|c|c|c|}
\hline $\begin{array}{l}\text { Sample } \\
\text { identifier }\end{array}$ & $\begin{array}{l}\text { Date sampled } \\
\qquad(\mathrm{m} / \mathrm{d} / \mathrm{y})\end{array}$ & Chromium & Iron & Lead & Mercury & Nickel & Silver \\
\hline \multirow[t]{5}{*}{ USGS 15} & $12 / 1 / 92$ & 13 & 80 & $<1$ & $<.1$ & 3 & $<1$ \\
\hline & $4 / 13 / 93$ & 6 & 70 & $<1$ & $<.1$ & $<1$ & $<1$ \\
\hline & $6 / 14 / 93$ & 7 & $<10$ & $<1$ & $<.1$ & $<1$ & $<1$ \\
\hline & $9 / 16 / 93$ & 6 & 20 & $<1$ & $<.1$ & $<1$ & $<1$ \\
\hline & $11 / 5 / 93$ & 6 & 30 & $<1$ & $<.1$ & 2 & $<1$ \\
\hline USGS 17 & $3 / 13 / 91$ & 3 & 130 & $<1$ & $<.1$ & 1 & $<1$ \\
\hline QAS-14 & $3 / 13 / 91$ & 4 & 110 & 1 & $<.1$ & 1 & $<1$ \\
\hline \multirow[t]{9}{*}{ USGS 17} & $6 / 6 / 91$ & 1 & 140 & 2 & $<.1$ & $<1$ & $<1$ \\
\hline & $9 / 5 / 91$ & 1 & 210 & $<1$ & $<.1$ & 2 & $<1$ \\
\hline & $12 / 3 / 91$ & $<1$ & 270 & $<1$ & $<.1$ & 2 & $<1$ \\
\hline & $3 / 16 / 92$ & $<1$ & 360 & $<1$ & $<.1$ & 2 & $<1$ \\
\hline & $6 / 11 / 92$ & $<1$ & 90 & $<1$ & $<.1$ & 1 & $<1$ \\
\hline & $9 / 16 / 92$ & 5 & 130 & 1 & $<.1$ & $<1$ & $<1$ \\
\hline & $12 / 3 / 92$ & $<1$ & 90 & $<1$ & $<.1$ & $<1$ & $<1$ \\
\hline & $4 / 7 / 93$ & 1 & 130 & $<1$ & $<.1$ & $<1$ & $<1$ \\
\hline & $6 / 11 / 93$ & $<1$ & 50 & $<1$ & $<.1$ & $<1$ & $<1$ \\
\hline QAS-29 & $6 / 11 / 93$ & 1 & 30 & $<1$ & $<.1$ & $<1$ & $<1$ \\
\hline \multirow[t]{2}{*}{ USGS 17} & $9 / 15 / 93$ & $<1$ & 70 & $<1$ & $<.1$ & $<1$ & $<1$ \\
\hline & $11 / 8 / 93$ & $<1$ & 80 & $<1$ & $<.1$ & 2 & $<1$ \\
\hline \multirow[t]{2}{*}{ USGS 97} & $3 / 13 / 91$ & 8 & 110 & 2 & $<.1$ & 2 & $<1$ \\
\hline & $6 / 7 / 91$ & 5 & 30 & 3 & $<.1$ & $<1$ & $<1$ \\
\hline QAS-15 & $6 / 7 / 91$ & 8 & 20 & 3 & $<.1$ & $<1$ & $<1$ \\
\hline \multirow[t]{8}{*}{ USGS 97} & 9/5/91 & 9 & 150 & 3 & $<.1$ & $<1$ & $<1$ \\
\hline & $12 / 3 / 91$ & 7 & 260 & 6 & $<.1$ & 2 & $<1$ \\
\hline & $3 / 16 / 92$ & 6 & 90 & $<1$ & $<.1$ & $<1$ & $<1$ \\
\hline & $6 / 17 / 92$ & 5 & 60 & 1 & $<.1$ & $<1$ & $<1$ \\
\hline & $9 / 21 / 92$ & 6 & 230 & 3 & $<.1$ & $<1$ & $<1$ \\
\hline & $12 / 8 / 92$ & 3 & 140 & 2 & $<.1$ & 2 & $<1$ \\
\hline & $4 / 6 / 93$ & 6 & 50 & 2 & $<.1$ & $<1$ & $<1$ \\
\hline & 6/9/93 & 5 & 20 & $<1$ & $<.1$ & $<1$ & $<1$ \\
\hline
\end{tabular}


Table 5.-Concentrations of selected total recoverable trace elements in water from roundthree samples, Naval Reactors Facility and vicinity-Continued

\begin{tabular}{|c|c|c|c|c|c|c|c|}
\hline $\begin{array}{l}\text { Sample } \\
\text { identifier }\end{array}$ & $\begin{array}{l}\text { Date sampled } \\
\qquad(\mathrm{m} / \mathrm{d} / \mathrm{y})\end{array}$ & Chromium & Iron & Lead & Mercury & Nickel & Silver \\
\hline \multirow[t]{2}{*}{ USGS 97} & $9 / 13 / 93$ & 6 & 110 & 1 & $<.1$ & $<1$ & $<1$ \\
\hline & $11 / 4 / 93$ & 7 & 730 & 2 & $<.1$ & SR & $<1$ \\
\hline QAS-32 & $11 / 4 / 93$ & 13 & 390 & 3 & $<.1$ & SR & $<1$ \\
\hline \multirow[t]{7}{*}{ USGS 98} & $3 / 13 / 91$ & 8 & 110 & 2 & $<.1$ & $<1$ & $<1$ \\
\hline & $6 / 7 / 91$ & 5 & 100 & 2 & $<.1$ & $<1$ & $<1$ \\
\hline & $9 / 5 / 91$ & 7 & 60 & 2 & $<.1$ & 2 & $<1$ \\
\hline & $12 / 3 / 91$ & 5 & 70 & $<1$ & $<.1$ & 1 & $<1$ \\
\hline & $3 / 16 / 92$ & 4 & 80 & $<1$ & $<.1$ & $<1$ & $<1$ \\
\hline & $6 / 16 / 92$ & 3 & 30 & 1 & $<.1$ & $<1$ & $<1$ \\
\hline & $9 / 21 / 92$ & 5 & 80 & 1 & $<.1$ & $<1$ & $<1$ \\
\hline QAS-25 & $9 / 21 / 92$ & 4 & 70 & 1 & $<.1$ & $<1$ & $<1$ \\
\hline \multirow[t]{3}{*}{ USGS 98} & $12 / 8 / 92$ & 9 & 140 & $<1$ & $<.1$ & 2 & $<1$ \\
\hline & $4 / 6 / 93$ & 4 & 130 & 1 & $<.1$ & $<1$ & $<1$ \\
\hline & $6 / 8 / 93$ & 3 & 50 & 2 & $<.1$ & $<1$ & $<1$ \\
\hline \multirow[t]{6}{*}{ USGS 99} & $3 / 13 / 91$ & 9 & 1,000 & 4 & $<.1$ & 2 & $<1$ \\
\hline & $6 / 7 / 91$ & 3 & 90 & 4 & $<.1$ & $<1$ & $<1$ \\
\hline & $9 / 5 / 91$ & 5 & 150 & 3 & $<.1$ & 1 & $<1$ \\
\hline & $12 / 3 / 91$ & 5 & 200 & $<1$ & $<.1$ & 2 & $<1$ \\
\hline & $3 / 16 / 92$ & 5 & 90 & $<1$ & $<.1$ & $<1$ & $<1$ \\
\hline & $6 / 16 / 92$ & 3 & 40 & 1 & $<.1$ & $<1$ & $<1$ \\
\hline QAS-24 & $6 / 16 / 92$ & 2 & 70 & 2 & $<.1$ & 1 & $<1$ \\
\hline \multirow[t]{6}{*}{ USGS 99} & $9 / 21 / 92$ & 5 & 160 & 2 & $<.1$ & 1 & $<1$ \\
\hline & $12 / 8 / 92$ & 8 & 160 & 1 & $<.1$ & 2 & $<1$ \\
\hline & $4 / 6 / 93$ & 4 & 130 & 1 & $<.1$ & $<1$ & $<1$ \\
\hline & $6 / 9 / 93$ & 5 & 80 & 1 & $<.1$ & $<1$ & $<1$ \\
\hline & $9 / 13 / 93$ & 6 & 70 & 1 & $<.1$ & $<1$ & $<1$ \\
\hline & $11 / 2 / 93$ & 5 & 70 & 2 & $<.1$ & 2 & $<1$ \\
\hline \multirow[t]{3}{*}{ USGS 102} & $6 / 7 / 91$ & 6 & 100 & 1 & $<.1$ & $<1$ & $<1$ \\
\hline & 9/5/91 & 7 & 160 & $<1$ & $<.1$ & 2 & $<1$ \\
\hline & $12 / 3 / 91$ & 7 & 2,100 & 2 & $<.1$ & 3 & $<1$ \\
\hline
\end{tabular}


Table 5.-Concentrations of selected total recoverable trace elements in water from roundthree samples, Naval Reactors Facility and vicinity-Continued

\begin{tabular}{|c|c|c|c|c|c|c|c|}
\hline $\begin{array}{c}\text { Sample } \\
\text { identifier }\end{array}$ & $\begin{array}{l}\text { Date sampled } \\
(\mathrm{m} / \mathrm{d} / \mathrm{y})\end{array}$ & Chromium & Iron & Lead & Mercury & Nickel & Silver \\
\hline \multirow[t]{4}{*}{ USGS 102} & $3 / 16 / 92$ & 6 & 130 & $<1$ & $<.1$ & $<1$ & $<1$ \\
\hline & $6 / 16 / 92$ & 6 & 90 & $<1$ & $<.1$ & 1 & $<1$ \\
\hline & $9 / 21 / 92$ & 6 & 110 & $<1$ & $<.1$ & $<1$ & $<1$ \\
\hline & $12 / 9 / 92$ & 4 & 30 & $<1$ & $<.1$ & 2 & $<1$ \\
\hline QAS-27 & $12 / 9 / 92$ & 8 & 40 & $<1$ & $<.1$ & 2 & $<1$ \\
\hline \multirow[t]{4}{*}{ USGS 102} & $4 / 6 / 93$ & 7 & 1,200 & $<1$ & $<.1$ & 1 & $<1$ \\
\hline & $6 / 9 / 93$ & 5 & 70 & $<1$ & $<.1$ & $<1$ & $<1$ \\
\hline & $9 / 13 / 93$ & 6 & 190 & $<1$ & $<.1$ & $<1$ & $<1$ \\
\hline & $11 / 4 / 93$ & 6 & 440 & $<1$ & $<.1$ & SR & $<1$ \\
\hline \multirow{4}{*}{$\begin{array}{l}\text { Water Supply } \\
\text { INEL-1 }\end{array}$} & $3 / 13 / 91$ & 11 & 200 & 2 & $<.1$ & 2 & $<1$ \\
\hline & $6 / 5 / 91$ & 10 & 90 & 2 & $<.1$ & $<1$ & $<1$ \\
\hline & $9 / 5 / 91$ & 10 & 250 & $<1$ & $<.1$ & 2 & $<1$ \\
\hline & $12 / 3 / 91$ & 9 & 200 & 3 & $<.1$ & 1 & $<1$ \\
\hline QAS-19 & $12 / 3 / 91$ & 7 & 170 & $<1$ & $<.1$ & $<1$ & $<1$ \\
\hline \multirow{8}{*}{$\begin{array}{l}\text { Water Supply } \\
\text { INEL-1 }\end{array}$} & $3 / 16 / 92$ & 7 & 150 & $<1$ & $<.1$ & $<1$ & $<1$ \\
\hline & $6 / 16 / 92$ & 8 & 520 & 4 & $<.1$ & 1 & $<1$ \\
\hline & $9 / 21 / 92$ & 10 & 770 & 5 & $<.1$ & 2 & $<1$ \\
\hline & $12 / 8 / 92$ & 14 & 430 & 1 & $<.1$ & 1 & $<1$ \\
\hline & $4 / 6 / 93$ & 10 & 340 & 1 & $<.1$ & $<1$ & $<1$ \\
\hline & $6 / 8 / 93$ & 8 & 280 & 5 & $<.1$ & $<1$ & $<1$ \\
\hline & $9 / 13 / 93$ & 7 & 310 & 1 & $<.1$ & $<1$ & $<1$ \\
\hline & $11 / 2 / 93$ & 10 & 2.000 & 2 & $<.1$ & 2 & $<1$ \\
\hline
\end{tabular}


Table 6.- Statistical parameters for selected total recoverable trace elements, by well

[See figure 2 for location of wells. Units are micrograms per liter. Symbol: < indicates concentration is less than the specified laboratory reporting level. Values are derived from tables 5,12 , and 18. Quality-assurance replicates are included in the calculation of statistical parameters. Mean and median sample size: includes all samples with concentrations greater than laboratory reporting level]

\begin{tabular}{|c|c|c|c|c|c|c|}
\hline \multirow{2}{*}{ Constituent } & \multicolumn{6}{|c|}{ Statistical parameter } \\
\hline & Minimum & Maximum & Median & Mean & Sample size & $\begin{array}{l}\text { Mean and median } \\
\text { sample size }\end{array}$ \\
\hline & \multicolumn{6}{|c|}{ NRF-1 } \\
\hline Chromium & 6 & 21 & 9 & 10 & 13 & 13 \\
\hline Iron & 30 & 3,200 & 190 & 528 & 13 & 13 \\
\hline Lead & $<1$ & 4 & 2 & 2 & 13 & 7 \\
\hline \multirow[t]{2}{*}{ Nickel } & $<1$ & 6 & 2 & 2 & 12 & 7 \\
\hline & \multicolumn{6}{|c|}{ NRF-2 } \\
\hline Chromium & $<1$ & 15 & 11 & 11 & 14 & 13 \\
\hline Iron & $<10$ & 80 & 20 & 27 & 14 & 9 \\
\hline Lead & $<1$ & 4 & 2.5 & 2 & 14 & 2 \\
\hline \multirow[t]{2}{*}{ Nickel } & $<1$ & 5 & 2 & 3 & 13 & 4 \\
\hline & \multicolumn{6}{|c|}{ NRF-3 } \\
\hline Chromium & 4 & 13 & 7 & 7 & 13 & 13 \\
\hline Iron & 30 & 4,900 & 140 & 608 & 13 & 13 \\
\hline Lead & $<1$ & 3 & 2 & 2 & 13 & 6 \\
\hline \multirow[t]{2}{*}{ Nickel } & $<1$ & 14 & 2.5 & 4 & 12 & 6 \\
\hline & \multicolumn{6}{|c|}{ NRF-4 } \\
\hline Chromium & 6 & 12 & 9 & 9 & 13 & 13 \\
\hline Iron & $<10$ & 300 & 55 & 91 & 13 & 8 \\
\hline Lead & $<1$ & 1 & 1 & 1 & 13 & 2 \\
\hline \multirow[t]{2}{*}{ Nickel } & $<1$ & 2 & 2 & 2 & 12 & 2 \\
\hline & \multicolumn{6}{|c|}{ NRF-6 } \\
\hline Arsenic & 3 & 4 & 3 & 3 & 7 & 7 \\
\hline Barium & $<100$ & 200 & 100 & 125 & 7 & 4 \\
\hline Chromium & $<1$ & 45 & 38.5 & 36 & 12 & 10 \\
\hline Copper & $<1$ & 6 & 5 & 4 & 5 & 3 \\
\hline Iron & 70 & 1,100 & 120 & 238 & 12 & 12 \\
\hline
\end{tabular}


Table 6.-Statistical parameters for selected total recoverable trace elements, by well-Continued

\begin{tabular}{|c|c|c|c|c|c|c|}
\hline \multirow[b]{2}{*}{ Constituent } & \multicolumn{6}{|c|}{ Statistical parameter } \\
\hline & Minimum & Maximum & Median & Mean & Sample size & $\begin{array}{l}\text { Mean and median } \\
\text { sample size }\end{array}$ \\
\hline & \multicolumn{6}{|c|}{ NRF-6 - cont. } \\
\hline Manganese & $<10$ & 40 & 30 & 30 & 7 & 4 \\
\hline Nickel & 5 & 48 & 13 & 16 & 11 & 11 \\
\hline Selenium & 1 & 3 & 2 & 2 & 7 & 7 \\
\hline \multirow[t]{2}{*}{ Zinc } & $<10$ & 30 & 10 & 16 & 7 & 5 \\
\hline & \multicolumn{6}{|c|}{ NRF-7 } \\
\hline Arsenic & $<1$ & 2 & 2 & 2 & 7 & 6 \\
\hline Barium & $<100$ & 100 & 100 & 100 & 6 & 2 \\
\hline Chromium & $<1$ & 13 & 10 & 10 & 12 & 11 \\
\hline Copper & 2 & 4 & 2 & 3 & 3 & 3 \\
\hline Iron & 180 & 3,900 & 330 & 662 & 12 & 12 \\
\hline Lead & $<1$ & 4 & 3.5 & 4 & 12 & 2 \\
\hline Manganese & $<10$ & 40 & 20 & 27 & 6 & 3 \\
\hline Nickel & $<1$ & 9 & 5 & 5 & 11 & 10 \\
\hline Selenium & $<1$ & 2 & 1 & 1 & 7 & 5 \\
\hline \multirow[t]{2}{*}{ Zinc } & $<10$ & 40 & 30 & 27 & 6 & 3 \\
\hline & \multicolumn{6}{|c|}{ USGS 12} \\
\hline Chromium & 4 & 10 & 6 & 6 & 13 & 13 \\
\hline Iron & 20 & 3,000 & 70 & 322 & 13 & 13 \\
\hline \multirow[t]{2}{*}{ Nickel } & $<1$ & 7 & 1.5 & 2 & 13 & 10 \\
\hline & \multicolumn{6}{|c|}{ USGS 15} \\
\hline Chromium & $<1$ & 71 & 6 & 13 & 12 & 11 \\
\hline Iron & $<10$ & 30,000 & 40 & 3,390 & 12 & 9 \\
\hline Lead & $<1$ & 16 & 9 & 9 & 12 & 2 \\
\hline \multirow[t]{2}{*}{ Nickel } & $<1$ & 86 & 2 & 14 & 12 & 7 \\
\hline & \multicolumn{6}{|c|}{ USGS 17} \\
\hline Chromium & $<1$ & 5 & 1 & 2 & 14 & 7 \\
\hline Iron & 30 & 360 & 120 & 135 & 14 & 14 \\
\hline Lead & $<1$ & 2 & 1 & 1 & 14 & 3 \\
\hline Nickel & $<1$ & 2 & 2 & 2 & 14 & 7 \\
\hline
\end{tabular}


Table 6.-Statistical parameters for selected total recoverable trace elements, by well-Continued

\begin{tabular}{|c|c|c|c|c|c|c|}
\hline \multirow[b]{2}{*}{ Constituent } & \multicolumn{6}{|c|}{ Statistical parameter } \\
\hline & Minimum & Maximum & Median & Mean & Sample size & $\begin{array}{l}\text { Mean and median } \\
\text { sample size }\end{array}$ \\
\hline & \multicolumn{6}{|c|}{ USGS 97} \\
\hline Chromium & 3 & 13 & 6 & 7 & 14 & 14 \\
\hline Iron & 20 & 730 & 110 & 171 & 14 & 14 \\
\hline Lead & $<1$ & 6 & 2.5 & 3 & 14 & 12 \\
\hline \multirow[t]{2}{*}{ Nickel } & $<1$ & 2 & 2 & 2 & 12 & 3 \\
\hline & \multicolumn{6}{|c|}{ USGS 98} \\
\hline Chromium & 3 & 9 & 5 & 5 & 11 & 11 \\
\hline Iron & 30 & 140 & 80 & 84 & 11 & 11 \\
\hline Lead & $<1$ & 2 & 1.5 & 2 & 11 & 8 \\
\hline \multirow[t]{2}{*}{ Nickel } & $<1$ & 2 & 2 & 2 & 11 & 3 \\
\hline & \multicolumn{6}{|c|}{ USGS 99} \\
\hline Chromium & 2 & 9 & 5 & 5 & 13 & 13 \\
\hline Iron & 40 & 1,000 & 90 & 178 & 13 & 13 \\
\hline Lead & $<1$ & 4 & 2 & 2 & 13 & 11 \\
\hline \multirow[t]{2}{*}{ Nickel } & $<1$ & 2 & 2 & 2 & 13 & 7 \\
\hline & \multicolumn{6}{|c|}{ USGS 102} \\
\hline Chromium & 4 & 8 & 6 & 6 & 12 & 12 \\
\hline Iron & 30 & 2,100 & 120 & 388 & 12 & 12 \\
\hline Lead & $<1$ & 2 & 1.5 & 2 & 12 & 2 \\
\hline \multirow[t]{2}{*}{ Nickel } & $<1$ & 3 & 2 & 2 & 11 & 6 \\
\hline & \multicolumn{6}{|c|}{ Water Supply INEL-1 } \\
\hline Chromium & 7 & 14 & 10 & 9 & 13 & 13 \\
\hline Iron & 90 & 2,000 & 280 & 439 & 13 & 13 \\
\hline Lead & $<1$ & 5 & 2 & 3 & 13 & 10 \\
\hline Nickel & $<1$ & 2 & 2 & 2 & 13 & 7 \\
\hline
\end{tabular}


Table 7.-Concentrations of dissolved nutrients and total organic carbon in water from round-three samples, Naval Reactors Facility and vicinity

[Analyses were performed by the U.S. Geological Survey's National Water Quality Laboratory. Analytical results are in milligrams per liter. Abbreviations: (m/d/y), month/day/year. Symbols: $<$ indicates concentration is less than the specified laboratory reporting level; NR indicates analysis not requested; BB indicates bottle broke during shipment. Sample identifier: see figure 2 for location of wells; QAS indicates quality-assurance sample, 23 and 30 are blank samples, others are replicates]

\begin{tabular}{|c|c|c|c|c|c|c|c|}
\hline $\begin{array}{c}\text { Sample } \\
\text { identifier }\end{array}$ & $\begin{array}{c}\text { Date } \\
\text { sampled } \\
(\mathrm{m} / \mathrm{d} / \mathrm{y})\end{array}$ & $\begin{array}{c}\text { Ammonia } \\
\text { (as } \\
\text { nitrogen) }\end{array}$ & $\begin{array}{c}\text { Ammonia } \\
\text { plus organic } \\
\text { nitrogen (as } \\
\text { nitrogen) }\end{array}$ & $\begin{array}{l}\text { Nitrite } \\
\text { (as } \\
\text { nitrogen) }\end{array}$ & $\begin{array}{c}\text { Nitrite plus } \\
\text { nitrate (as } \\
\text { nitrogen) }\end{array}$ & $\begin{array}{c}\text { Ortho- } \\
\text { phosphate } \\
\text { (as } \\
\text { phosphorus) }\end{array}$ & $\begin{array}{l}\text { Total } \\
\text { organic } \\
\text { carbon }\end{array}$ \\
\hline QAS-23 & $6 / 12 / 92$ & $<0.01$ & NR & $<0.01$ & $<0.05$ & $<0.01$ & $<0.1$ \\
\hline QAS-30 & $6 / 15 / 93$ & .02 & NR & $<.01$ & $<.05$ & $<.01$ & NR \\
\hline \multirow[t]{3}{*}{ NRF-1 } & $3 / 5 / 91$ & NR & .6 & $<.01$ & 1.6 & .02 & .5 \\
\hline & 6/17/91 & NR & .2 & $<.01$ & 1.8 & .02 & .4 \\
\hline & 9/9/91 & NR & .3 & .01 & 1.7 & $<.01$ & .4 \\
\hline QAS-18 & 9/9/91 & NR & .3 & $<.01$ & 1.6 & $<.01$ & .4 \\
\hline \multirow[t]{9}{*}{ NRF-1 } & $12 / 4 / 91$ & .01 & NR & $<.01$ & 1.7 & .02 & .5 \\
\hline & $3 / 11 / 92$ & .08 & NR & $<.01$ & 1.8 & .02 & 1.0 \\
\hline & $6 / 11 / 92$ & $<.01$ & NR & $<.01$ & 1.8 & .02 & .3 \\
\hline & 9/16/92 & $<.01$ & NR & $<.01$ & 1.8 & .02 & .9 \\
\hline & $12 / 3 / 92$ & $<.01$ & NR & $<.01$ & 1.9 & .02 & .7 \\
\hline & $4 / 7 / 93$ & $<.01$ & NR & $<.01$ & 1.8 & .02 & 1.0 \\
\hline & $6 / 15 / 93$ & .03 & NR & $<.01$ & 1.9 & .02 & .5 \\
\hline & $9 / 15 / 93$ & .02 & NR & $<.01$ & 1.9 & .03 & .4 \\
\hline & $11 / 4 / 93$ & .02 & NR & $<.01$ & 1.9 & .04 & .7 \\
\hline NRF-2 & $3 / 5 / 91$ & NR & .3 & $<.01$ & 1.9 & .02 & .5 \\
\hline QAS-13 & $3 / 5 / 91$ & NR & $<.2$ & $<.01$ & 1.9 & .02 & .4 \\
\hline \multirow[t]{6}{*}{ NRF-2 } & $6 / 17 / 91$ & NR & $<.2$ & $<.01$ & 2.0 & .02 & .4 \\
\hline & 9/9/91 & NR & .4 & $<.01$ & 1.8 & $<.01$ & .4 \\
\hline & $12 / 4 / 91$ & .01 & NR & $<.01$ & 1.9 & .02 & .6 \\
\hline & $3 / 11 / 92$ & .08 & NR & $<.01$ & 2.0 & .02 & .4 \\
\hline & $6 / 11 / 92$ & $<.01$ & NR & $<.01$ & 2.0 & .03 & .3 \\
\hline & $9 / 16 / 92$ & $<.01$ & NR & $<.01$ & 1.9 & .02 & .9 \\
\hline
\end{tabular}


Table 7.-Concentrations of dissolved nutrients and total organic carbon in water from round-three samples, Naval Reactors Facility and vicinity-Continued

\begin{tabular}{|c|c|c|c|c|c|c|c|}
\hline $\begin{array}{c}\text { Sample } \\
\text { identifier }\end{array}$ & $\begin{array}{c}\text { Date } \\
\text { sampled } \\
(\mathrm{m} / \mathrm{d} / \mathrm{y})\end{array}$ & $\begin{array}{l}\text { Ammonia } \\
\quad \text { (as } \\
\text { nitrogen) }\end{array}$ & $\begin{array}{c}\text { Ammonia } \\
\text { plus organic } \\
\text { nitrogen (as } \\
\text { nitrogen) }\end{array}$ & $\begin{array}{c}\text { Nitrite } \\
\text { (as } \\
\text { nitrogen) }\end{array}$ & $\begin{array}{c}\text { Nitrite plus } \\
\text { nitrate (as } \\
\text { nitrogen) }\end{array}$ & $\begin{array}{c}\text { Ortho- } \\
\text { phosphate } \\
\text { (as } \\
\text { phosphorus) }\end{array}$ & $\begin{array}{l}\text { Total } \\
\text { organic } \\
\text { carbon }\end{array}$ \\
\hline \multirow[t]{4}{*}{ NRF-2 } & $12 / 3 / 92$ & $<.01$ & NR & $<.01$ & 2.0 & .02 & .5 \\
\hline & $4 / 7 / 93$ & $<.01$ & NR & $<.01$ & 1.9 & .02 & .6 \\
\hline & $6 / 15 / 93$ & .03 & NR & $<.01$ & 2.0 & .02 & .4 \\
\hline & $9 / 15 / 93$ & .02 & NR & $<.01$ & 2.0 & .03 & .5 \\
\hline QAS-31 & $9 / 15 / 93$ & .02 & NR & $<.01$ & 2.0 & .02 & 1.8 \\
\hline NRF-2 & $11 / 4 / 93$ & .01 & NR & $<.01$ & 2.0 & .03 & .4 \\
\hline NRF-3 & $6 / 17 / 91$ & NR & .4 & $<.01$ & 1.8 & .03 & 1.8 \\
\hline QAS-16 & $6 / 17 / 91$ & NR & .2 & $<.01$ & 1.8 & .02 & .5 \\
\hline \multirow[t]{6}{*}{ NRF-3 } & $9 / 26 / 91$ & NR & $<.2$ & $<.01$ & 1.8 & .03 & .5 \\
\hline & $12 / 4 / 91$ & .01 & NR & $<.01$ & 1.7 & .02 & .6 \\
\hline & $3 / 11 / 92$ & .07 & NR & $<.01$ & 1.8 & .02 & .5 \\
\hline & $6 / 11 / 92$ & $<.01$ & NR & $<.01$ & 1.8 & .02 & .7 \\
\hline & $9 / 16 / 92$ & .02 & NR & $<.01$ & 1.8 & .02 & 1.8 \\
\hline & $12 / 3 / 92$ & $<.01$ & NR & .01 & 1.9 & .02 & .7 \\
\hline QAS-26 & $12 / 3 / 92$ & $<.01$ & NR & $<.01$ & 1.9 & .02 & .7 \\
\hline \multirow[t]{4}{*}{ NRF-3 } & $4 / 8 / 93$ & $<.01$ & NR & $<.01$ & 1.8 & .02 & 1.9 \\
\hline & $6 / 15 / 93$ & .03 & NR & $<.01$ & 1.8 & .02 & 1.4 \\
\hline & $9 / 15 / 93$ & .02 & NR & $<.01$ & 1.9 & .02 & 1.0 \\
\hline & $11 / 4 / 93$ & .01 & NR & $<.01$ & 1.9 & .02 & 1.2 \\
\hline \multirow[t]{8}{*}{ NRF-4 } & $3 / 5 / 91$ & NR & .3 & $<.01$ & 1.9 & .03 & .4 \\
\hline & $6 / 17 / 91$ & NR & .2 & $<.01$ & 1.9 & .02 & .3 \\
\hline & 9/9/91 & NR & .4 & $<.01$ & 1.9 & $<.01$ & .4 \\
\hline & $12 / 4 / 91$ & .02 & NR & $<.01$ & 1.9 & .02 & .7 \\
\hline & $3 / 11 / 92$ & .07 & NR & $<.01$ & 2.1 & .03 & .5 \\
\hline & $6 / 11 / 92$ & $<.01$ & NR & $<.01$ & 2.0 & .03 & .4 \\
\hline & $9 / 16 / 92$ & .01 & NR & $<.01$ & 2.0 & .02 & .9 \\
\hline & $12 / 3 / 92$ & $<.01$ & NR & .01 & 2.1 & .02 & .4 \\
\hline
\end{tabular}


Table 7.-Concentrations of dissolved nutrients and total organic carbon in water from round-three samples, Naval Reactors Facility and vicinity-Continued

\begin{tabular}{|c|c|c|c|c|c|c|c|}
\hline $\begin{array}{l}\text { Sample } \\
\text { identifier }\end{array}$ & $\begin{array}{c}\text { Date } \\
\text { sampled } \\
(\mathrm{m} / \mathrm{d} / \mathrm{y})\end{array}$ & $\begin{array}{l}\text { Ammonia } \\
\quad \text { (as } \\
\text { nitrogen) }\end{array}$ & $\begin{array}{c}\text { Ammonia } \\
\text { plus organic } \\
\text { nitrogen (as } \\
\text { nitrogen) }\end{array}$ & $\begin{array}{l}\text { Nitrite } \\
\quad \text { (as } \\
\text { nitrogen) }\end{array}$ & $\begin{array}{c}\text { Nitrite plus } \\
\text { nitrate (as } \\
\text { nitrogen) }\end{array}$ & $\begin{array}{c}\text { Ortho- } \\
\text { phosphate } \\
\text { (as } \\
\text { phosphorus) }\end{array}$ & $\begin{array}{l}\text { Total } \\
\text { organic } \\
\text { carbon }\end{array}$ \\
\hline NRF-4 & $4 / 7 / 93$ & $<.01$ & NR & $<.01$ & 2.0 & .02 & 1.0 \\
\hline QAS-28 & $4 / 7 / 93$ & $<.01$ & NR & $<.01$ & 2.0 & .02 & .5 \\
\hline \multirow[t]{3}{*}{ NRF-4 } & $6 / 15 / 93$ & .03 & NR & $<.01$ & 2.1 & .02 & .5 \\
\hline & $9 / 15 / 93$ & .02 & NR & $<.01$ & 2.2 & .03 & 1.4 \\
\hline & $11 / 4 / 93$ & .02 & NR & $<.01$ & 2.2 & .02 & .6 \\
\hline \multirow[t]{5}{*}{ NRF-6 } & $9 / 18 / 92$ & .01 & NR & $<.01$ & 1.7 & .08 & .9 \\
\hline & $12 / 9 / 92$ & $<.01$ & NR & .01 & 1.8 & .07 & .6 \\
\hline & $4 / 9 / 93$ & $<.01$ & NR & $<.01$ & 1.9 & .08 & 1.2 \\
\hline & $9 / 14 / 93$ & .01 & NR & $<.01$ & 1.8 & .07 & BB \\
\hline & $11 / 4 / 93$ & .02 & NR & $<.01$ & 1.9 & .07 & .6 \\
\hline \multirow[t]{6}{*}{ NRF-7 } & $9 / 18 / 92$ & .02 & NR & $<.01$ & 1.8 & .02 & .8 \\
\hline & $12 / 9 / 92$ & $<.01$ & NR & .03 & .54 & .02 & .1 \\
\hline & $4 / 9 / 93$ & $<.01$ & NR & $<.01$ & .47 & .01 & .5 \\
\hline & $6 / 10 / 93$ & .02 & NR & $<.01$ & .48 & .03 & .2 \\
\hline & $9 / 14 / 93$ & .02 & NR & $<.01$ & .53 & .02 & .1 \\
\hline & $11 / 3 / 93$ & .03 & NR & $<.01$ & .53 & .02 & .1 \\
\hline \multirow[t]{2}{*}{ USGS 12} & $6 / 10 / 91$ & NR & $<.2$ & .01 & 1.8 & .01 & .3 \\
\hline & 9/6/91 & NR & .2 & $<.01$ & 1.8 & $<.01$ & .4 \\
\hline QAS-17 & 9/6/91 & NR & .3 & $<.01$ & 1.7 & $<.01$ & .4 \\
\hline \multirow[t]{8}{*}{ USGS 12} & $12 / 5 / 91$ & .01 & NR & $<.01$ & 1.8 & .02 & .5 \\
\hline & $3 / 12 / 92$ & .06 & NR & $<.01$ & 1.9 & .02 & .4 \\
\hline & $6 / 19 / 92$ & .01 & NR & $<.01$ & 2.0 & .04 & .3 \\
\hline & $9 / 18 / 92$ & .01 & NR & $<.01$ & .46 & .01 & .6 \\
\hline & $12 / 1 / 92$ & $<.01$ & NR & .02 & 2.0 & .02 & .4 \\
\hline & $4 / 13 / 93$ & $<.01$ & NR & $<.01$ & 2.0 & .02 & .4 \\
\hline & $6 / 14 / 93$ & .02 & NR & $<.01$ & 2.1 & .03 & .6 \\
\hline & $9 / 16 / 93$ & .02 & NR & $<.01$ & 2.0 & .03 & 1.7 \\
\hline
\end{tabular}


Table 7.-Concentrations of dissolved nutrients and total organic carbon in water from round-three samples, Naval Reactors Facility and vicinity-Continued

\begin{tabular}{|c|c|c|c|c|c|c|c|}
\hline $\begin{array}{l}\text { Sample } \\
\text { identifier }\end{array}$ & $\begin{array}{c}\text { Date } \\
\text { sampled } \\
(\mathrm{m} / \mathrm{d} / \mathrm{y})\end{array}$ & $\begin{array}{l}\text { Ammonia } \\
\quad \text { (as } \\
\text { nitrogen) }\end{array}$ & $\begin{array}{c}\text { Ammonia } \\
\text { plus organic } \\
\text { nitrogen (as } \\
\text { nitrogen) }\end{array}$ & $\begin{array}{l}\text { Nitrite } \\
\quad \text { (as } \\
\text { nitrogen) }\end{array}$ & $\begin{array}{c}\text { Nitrite plus } \\
\text { nitrate (as } \\
\text { nitrogen) }\end{array}$ & $\begin{array}{c}\text { Ortho- } \\
\text { phosphate } \\
\text { (as } \\
\text { phosphorus) }\end{array}$ & $\begin{array}{l}\text { Total } \\
\text { organic } \\
\text { carbon }\end{array}$ \\
\hline USGS 12 & $11 / 5 / 93$ & .02 & NR & $<.01$ & 2.0 & .03 & .4 \\
\hline QAS-33 & $11 / 5 / 93$ & .02 & NR & $<.01$ & 2.0 & .02 & 6 \\
\hline \multirow[t]{4}{*}{ USGS 15} & $6 / 10 / 91$ & NR & $<.02$ & $<.01$ & .85 & $<.01$ & .2 \\
\hline & $9 / 6 / 91$ & NR & $<.02$ & $<.01$ & 1.3 & $<.01$ & .3 \\
\hline & $12 / 4 / 91$ & .01 & NR & $<.01$ & .48 & .02 & .3 \\
\hline & $3 / 12 / 92$ & $<.01$ & NR & $<.01$ & .30 & .01 & $<.1$ \\
\hline QAS-22 & $3 / 12 / 92$ & .04 & NR & $<.01$ & .34 & .02 & .2 \\
\hline \multirow[t]{7}{*}{ USGS 15} & $6 / 19 / 92$ & .02 & NR & $<.01$ & .98 & .04 & 3.8 \\
\hline & $9 / 17 / 92$ & $<.01$ & NR & $<.01$ & .95 & .02 & .4 \\
\hline & $12 / 1 / 92$ & $<.01$ & NR & .03 & .45 & .02 & .4 \\
\hline & $4 / 13 / 93$ & $<.01$ & NR & $<.01$ & .32 & .01 & .4 \\
\hline & $6 / 14 / 93$ & .02 & NR & $<.01$ & .54 & .02 & .3 \\
\hline & $9 / 16 / 93$ & .03 & NR & $<.01$ & 1.0 & .03 & .2 \\
\hline & $11 / 5 / 93$ & .04 & NR & $<.01$ & .55 & .02 & .4 \\
\hline USGS 17 & $3 / 13 / 91$ & NR & $<.2$ & .02 & .34 & .01 & .1 \\
\hline QAS-14 & $3 / 13 / 91$ & NR & $<.2$ & .02 & .31 & .01 & .4 \\
\hline \multirow[t]{9}{*}{ USGS 17} & $6 / 6 / 91$ & NR & $<.2$ & $<.01$ & .32 & $<.01$ & .1 \\
\hline & $9 / 5 / 91$ & NR & $<.2$ & $<.01$ & .31 & .03 & .1 \\
\hline & $12 / 3 / 91$ & .01 & NR & $<.01$ & .33 & .01 & .4 \\
\hline & $3 / 16 / 92$ & $<.01$ & NR & $<.01$ & .30 & .01 & $<.1$ \\
\hline & $6 / 11 / 92$ & $<.01$ & NR & $<.01$ & .33 & .01 & .1 \\
\hline & $9 / 16 / 92$ & .01 & NR & $<.01$ & .34 & .01 & 1.0 \\
\hline & $12 / 3 / 92$ & $<.01$ & NR & $<.01$ & .36 & $<.01$ & .2 \\
\hline & $4 / 7 / 93$ & .04 & NR & $<.01$ & .31 & .02 & .4 \\
\hline & $6 / 11 / 93$ & .02 & NR & $<.01$ & .35 & .03 & .1 \\
\hline QAS-29 & $6 / 11 / 93$ & .02 & NR & $<.01$ & .34 & .02 & .5 \\
\hline USGS 17 & $9 / 15 / 93$ & .02 & NR & $<.01$ & .36 & .01 & .2 \\
\hline
\end{tabular}


Table 7.-Concentrations of dissolved nutrients and total organic carbon in water from round-three samples, Naval Reactors Facility and vicinity-Continued

\begin{tabular}{|c|c|c|c|c|c|c|c|}
\hline $\begin{array}{l}\text { Sample } \\
\text { identifier }\end{array}$ & $\begin{array}{c}\text { Date } \\
\text { sampled } \\
(\mathrm{m} / \mathrm{d} / \mathrm{y})\end{array}$ & $\begin{array}{l}\text { Ammonia } \\
\quad \text { (as } \\
\text { nitrogen) }\end{array}$ & $\begin{array}{l}\text { Ammonia } \\
\text { plus organic } \\
\text { nitrogen (as } \\
\text { nitrogen) }\end{array}$ & $\begin{array}{l}\text { Nitrite } \\
\quad \text { (as } \\
\text { nitrogen) }\end{array}$ & $\begin{array}{l}\text { Nitrite plus } \\
\text { nitrate (as } \\
\text { nitrogen) }\end{array}$ & $\begin{array}{c}\text { Ortho- } \\
\text { phosphate } \\
\text { (as } \\
\text { phosphorus) }\end{array}$ & $\begin{array}{l}\text { Total } \\
\text { organic } \\
\text { carbon }\end{array}$ \\
\hline USGS 17 & $11 / 8 / 93$ & $<.01$ & NR & $<.01$ & .4 & .02 & .1 \\
\hline \multirow[t]{2}{*}{ USGS 97} & $3 / 13 / 91$ & NR & $<.2$ & .02 & 1.9 & .02 & .3 \\
\hline & $6 / 7 / 91$ & NR & .2 & $<.01$ & 1.9 & .02 & .3 \\
\hline QAS-15 & $6 / 7 / 91$ & NR & .2 & $<.01$ & 2.0 & .01 & .4 \\
\hline \multirow[t]{10}{*}{ USGS 97} & $9 / 5 / 91$ & NR & .3 & $<.01$ & 2.0 & $<.01$ & .6 \\
\hline & $12 / 3 / 91$ & $<.01$ & NR & $<.01$ & 1.9 & .02 & .6 \\
\hline & $3 / 16 / 92$ & $<.01$ & NR & $<.01$ & 1.9 & .02 & .3 \\
\hline & $6 / 17 / 92$ & .02 & NR & $<.01$ & 2.0 & .02 & .8 \\
\hline & $9 / 21 / 92$ & .01 & NR & $<.01$ & 1.9 & .03 & .6 \\
\hline & $12 / 8 / 92$ & $<.01$ & NR & .01 & 2.1 & .03 & .4 \\
\hline & $4 / 6 / 93$ & $<.01$ & NR & $<.01$ & 2.0 & .02 & .5 \\
\hline & $6 / 9 / 93$ & .02 & NR & $<.01$ & 1.9 & .02 & .4 \\
\hline & 9/13/93 & .02 & NR & $<.01$ & 2.1 & .02 & .5 \\
\hline & $11 / 4 / 93$ & .01 & NR & $<.01$ & 2.0 & .03 & .4 \\
\hline QAS-32 & $11 / 4 / 93$ & .02 & NR & $<.01$ & 2.0 & .04 & .5 \\
\hline \multirow[t]{7}{*}{ USGS 98} & $3 / 13 / 91$ & NR & $<.2$ & $<.01$ & 1.1 & .02 & .1 \\
\hline & $6 / 7 / 91$ & NR & $<.2$ & $<.01$ & 1.1 & $<.01$ & .2 \\
\hline & 9/5/91 & NR & .4 & $<.01$ & 1.1 & $<.01$ & .2 \\
\hline & $12 / 3 / 91$ & .01 & NR & $<.01$ & 1.1 & .02 & .4 \\
\hline & $3 / 16 / 92$ & $<.01$ & NR & $<.01$ & 1.0 & .01 & $<.1$ \\
\hline & $6 / 16 / 92$ & .02 & NR & $<.01$ & 1.1 & .02 & .2 \\
\hline & $9 / 21 / 92$ & $<.01$ & NR & $<.01$ & 1.0 & .01 & .5 \\
\hline QAS-25 & $9 / 21 / 92$ & .02 & NR & $<.01$ & 1.0 & .02 & .5 \\
\hline \multirow[t]{3}{*}{ USGS 98} & $12 / 8 / 92$ & $<.01$ & NR & .02 & 1.1 & .02 & .3 \\
\hline & $4 / 6 / 93$ & .04 & NR & $<.01$ & 1.1 & .02 & 1.0 \\
\hline & $6 / 8 / 93$ & .01 & NR & $<.01$ & 1.0 & .02 & .2 \\
\hline USGS 99 & $3 / 13 / 91$ & NR & $<.2$ & .02 & 1.6 & .02 & .3 \\
\hline
\end{tabular}


Table 7.-Concentrations of dissolved nutrients and total organic carbon in water from round-three samples, Naval Reactors Facility and vicinity-Continued

\begin{tabular}{|c|c|c|c|c|c|c|c|}
\hline $\begin{array}{c}\text { Sample } \\
\text { identifier }\end{array}$ & $\begin{array}{c}\text { Date } \\
\text { sampled } \\
(\mathrm{m} / \mathrm{d} / \mathrm{y})\end{array}$ & $\begin{array}{l}\text { Ammonia } \\
\quad \text { (as } \\
\text { nitrogen) }\end{array}$ & $\begin{array}{c}\text { Ammonia } \\
\text { plus organic } \\
\text { nitrogen (as } \\
\text { nitrogen) }\end{array}$ & $\begin{array}{l}\text { Nitrite } \\
\text { (as } \\
\text { nitrogen) }\end{array}$ & $\begin{array}{c}\text { Nitrite plus } \\
\text { nitrate (as } \\
\text { nitrogen) }\end{array}$ & $\begin{array}{c}\text { Ortho- } \\
\text { phosphate } \\
\text { (as } \\
\text { phosphorus) }\end{array}$ & $\begin{array}{l}\text { Total } \\
\text { organic } \\
\text { carbon }\end{array}$ \\
\hline \multirow[t]{5}{*}{ USGS 99} & $6 / 7 / 91$ & NR & $<.2$ & $<.01$ & 1.6 & $<.01$ & .3 \\
\hline & $9 / 5 / 91$ & NR & .3 & $<.01$ & 1.5 & $<.01$ & .7 \\
\hline & $12 / 3 / 91$ & .01 & NR & $<.01$ & 1.5 & .02 & .4 \\
\hline & $3 / 16 / 92$ & $<.01$ & NR & $<.01$ & 1.5 & .02 & .1 \\
\hline & $6 / 16 / 92$ & .02 & NR & $<.01$ & 1.5 & .02 & .3 \\
\hline QAS-24 & $6 / 16 / 92$ & .01 & NR & $<.01$ & 1.5 & .02 & .4 \\
\hline \multirow[t]{6}{*}{ USGS 99} & $9 / 21 / 92$ & $<.01$ & NR & $<.01$ & 1.5 & .02 & .7 \\
\hline & $12 / 8 / 92$ & $<.01$ & NR & .01 & 1.6 & .02 & .6 \\
\hline & $4 / 6 / 93$ & $<.01$ & NR & $<.01$ & 1.5 & .02 & 1.7 \\
\hline & $6 / 9 / 93$ & .01 & NR & $<.01$ & 1.5 & .02 & .3 \\
\hline & $9 / 13 / 93$ & .02 & NR & $<.01$ & 1.6 & .01 & .5 \\
\hline & $11 / 2 / 93$ & .03 & NR & $<.01$ & 1.6 & .02 & .4 \\
\hline \multirow[t]{7}{*}{ USGS 102} & $6 / 7 / 91$ & NR & .2 & $<.01$ & 1.8 & .01 & .4 \\
\hline & $9 / 5 / 91$ & NR & $<.2$ & $<.01$ & 1.8 & $<.01$ & .2 \\
\hline & $12 / 3 / 91$ & $<.01$ & NR & $<.01$ & 1.8 & .02 & .5 \\
\hline & $3 / 16 / 92$ & $<.01$ & NR & $<.01$ & 1.7 & .02 & .2 \\
\hline & $6 / 16 / 92$ & .02 & NR & $<.01$ & 1.8 & .03 & .4 \\
\hline & $9 / 21 / 92$ & .02 & NR & $<.01$ & 1.8 & .02 & .8 \\
\hline & $12 / 9 / 92$ & $<.01$ & NR & .02 & 1.9 & .02 & .4 \\
\hline QAS-27 & $12 / 9 / 92$ & $<.01$ & NR & .02 & 1.9 & .02 & .4 \\
\hline \multirow[t]{4}{*}{ USGS 102} & $4 / 6 / 93$ & $<.01$ & NR & $<.01$ & 1.8 & .02 & .8 \\
\hline & $6 / 9 / 93$ & .01 & NR & $<.01$ & 1.8 & .02 & .4 \\
\hline & $9 / 13 / 93$ & .02 & NR & $<.01$ & 2.0 & .02 & .5 \\
\hline & $11 / 4 / 93$ & .01 & NR & $<.01$ & 2.0 & .03 & .4 \\
\hline \multirow{3}{*}{$\begin{array}{l}\text { Water Supply } \\
\text { INEL-1 }\end{array}$} & $3 / 13 / 91$ & NR & .4 & .02 & 5.2 & .01 & .7 \\
\hline & $6 / 5 / 91$ & NR & .5 & $<.01$ & 5.2 & $<.01$ & .5 \\
\hline & $9 / 5 / 91$ & NR & .4 & $<.01$ & 5.1 & $<.01$ & .9 \\
\hline
\end{tabular}


Table 7.-Concentrations of dissolved nutrients and total organic carbon in water from round-three samples, Naval Reactors Facility and vicinity-Continued

\begin{tabular}{lccccccc}
\hline \multicolumn{1}{c}{$\begin{array}{c}\text { Sample } \\
\text { identifier }\end{array}$} & $\begin{array}{c}\text { Date } \\
\text { sampled } \\
\text { (m/d/y) }\end{array}$ & $\begin{array}{c}\text { Ammonia } \\
\text { (as } \\
\text { nitrogen) }\end{array}$ & $\begin{array}{c}\text { Ammonia } \\
\text { plus organic } \\
\text { nitrogen (as } \\
\text { nitrogen) }\end{array}$ & $\begin{array}{c}\text { Nitrite } \\
\text { (as } \\
\text { nitrogen) }\end{array}$ & $\begin{array}{c}\text { Nitrite plus } \\
\text { nitrate (as } \\
\text { nitrogen) }\end{array}$ & $\begin{array}{c}\text { Ortho- } \\
\text { phosphate } \\
\text { (as } \\
\text { phosphorus) }\end{array}$ & $\begin{array}{c}\text { Total } \\
\text { organic } \\
\text { carbon }\end{array}$ \\
\hline $\begin{array}{l}\text { Water Supply } \\
\text { INEL-1 }\end{array}$ & $12 / 3 / 91$ & .02 & $\mathrm{NR}$ & $<.01$ & 5.1 & .01 & .9 \\
QAS-19 & $12 / 3 / 91$ & .02 & $\mathrm{NR}$ & $<.01$ & 5.4 & .01 & .7 \\
Water Supply & $3 / 16 / 92$ & $<.01$ & $\mathrm{NR}$ & $<.01$ & 4.5 & .01 & .5 \\
INEL-1 & $6 / 16 / 92$ & .02 & $\mathrm{NR}$ & $<.01$ & 5.0 & .01 & .8 \\
& $9 / 21 / 92$ & $<.01$ & $\mathrm{NR}$ & $<.01$ & 5.0 & .01 & 1.2 \\
& $12 / 8 / 92$ & $<.01$ & $\mathrm{NR}$ & .01 & 5.0 & .02 & .7 \\
& $4 / 6 / 93$ & $<.01$ & $\mathrm{NR}$ & $<.01$ & 4.5 & .02 & .8 \\
& $6 / 8 / 93$ & .01 & $\mathrm{NR}$ & $<.01$ & 4.6 & .01 & .6 \\
& $9 / 13 / 93$ & .02 & $\mathrm{NR}$ & $<.01$ & 4.6 & $<.01$ & .8 \\
& $11 / 2 / 93$ & .04 & $\mathrm{NR}$ & $<.01$ & 4.6 & .01 & .8 \\
\hline
\end{tabular}


Table 8. - Statistical parameters for dissolved nutrients, total organic carbon, total phenols, and turbidity, by well

[See figure 2 for location of wells. Units are milligrams per liter. Symbol: < indicates concentration is less than the specified laboratory reporting level. Values are derived from tables $7,12,13,18$, and 19. Quality-assurance replicates are included in the calculation of statistical parameters. Mean and median sample size: includes all samples with concentrations greater than laboratory reporting level]

\begin{tabular}{|c|c|c|c|c|c|c|}
\hline \multirow{2}{*}{$\begin{array}{l}\text { Constituent or physical } \\
\text { characteristic }\end{array}$} & \multicolumn{6}{|c|}{ Statistical parameter } \\
\hline & Minimum & Maximum & Median & Mean & $\begin{array}{l}\text { Sample } \\
\text { size }\end{array}$ & $\begin{array}{l}\text { Mean and median } \\
\text { sample size }\end{array}$ \\
\hline & \multicolumn{6}{|c|}{ NRF-1 } \\
\hline Ammonia (as nitrogen) & $<0.01$ & 0.08 & 0.02 & 0.03 & 9 & 5 \\
\hline $\begin{array}{l}\text { Ammonia plus organic } \\
\text { nitrogen (as nitrogen) }\end{array}$ & .2 & 6 & .3 & .4 & 4 & 4 \\
\hline $\begin{array}{l}\text { Nitrite plus nitrate } \\
\text { (as nitrogen) }\end{array}$ & 1.6 & 1.9 & 1.8 & 1.8 & 13 & 13 \\
\hline $\begin{array}{l}\text { Orthophosphate (as } \\
\text { phosphorus) }\end{array}$ & $<.01$ & .04 & .02 & .02 & 13 & 11 \\
\hline \multirow[t]{2}{*}{ Organic carbon } & .3 & 1.0 & .5 & .6 & 13 & 13 \\
\hline & \multicolumn{6}{|c|}{ NRF-2 } \\
\hline Ammonia (as nitrogen) & $<.01$ & .08 & .02 & .03 & 10 & 6 \\
\hline $\begin{array}{l}\text { Ammonia plus organic } \\
\text { nitrogen (as nitrogen) }\end{array}$ & $<.2$ & .4 & .35 & .4 & 4 & 2 \\
\hline $\begin{array}{l}\text { Nitrite plus nitrate } \\
\text { (as nitrogen) }\end{array}$ & 1.8 & 2.0 & 2.0 & 2.0 & 14 & 14 \\
\hline $\begin{array}{l}\text { Orthophosphate (as } \\
\text { phosphorus) }\end{array}$ & $<.01$ & .03 & .02 & .02 & 14 & 13 \\
\hline \multirow[t]{2}{*}{ Organic carbon } & .3 & 1.8 & .4 & .6 & 14 & 14 \\
\hline & \multicolumn{6}{|c|}{ NRF-3 } \\
\hline Ammonia (as nitrogen) & $<.01$ & .07 & .02 & .03 & 10 & 6 \\
\hline $\begin{array}{l}\text { Ammonia plus organic } \\
\text { nitrogen (as nitrogen) }\end{array}$ & $<.2$ & .4 & .3 & .3 & 3 & 2 \\
\hline $\begin{array}{l}\text { Nitrite plus nitrate } \\
\text { (as nitrogen) }\end{array}$ & 1.7 & 1.9 & 1.8 & 1.8 & 13 & 13 \\
\hline $\begin{array}{l}\text { Orthophosphate (as } \\
\text { phosphorus) }\end{array}$ & .02 & .03 & .02 & .02 & 13 & 13 \\
\hline \multirow[t]{2}{*}{ Organic carbon } & .5 & 1.9 & .7 & 1.0 & 13 & 13 \\
\hline & \multicolumn{6}{|c|}{ NRF-4 } \\
\hline Ammonia (as nitrogen) & $<.01$ & .07 & .02 & .03 & 10 & 6 \\
\hline
\end{tabular}


Table 8.-Statistical parameters for dissolved nutrients, total organic carbon, total phenols, and turbidity, by well-Continued

\begin{tabular}{|c|c|c|c|c|c|c|}
\hline \multirow{2}{*}{$\begin{array}{l}\text { Constituent or physical } \\
\text { characteristic }\end{array}$} & \multicolumn{6}{|c|}{ Statistical parameter } \\
\hline & Minimum & Maximum & Median & Mean & $\begin{array}{l}\text { Sample } \\
\text { size }\end{array}$ & $\begin{array}{l}\text { Mean and median } \\
\text { sample size }\end{array}$ \\
\hline & \multicolumn{6}{|c|}{ NRF-4 - cont. } \\
\hline $\begin{array}{l}\text { Ammonia plus organic } \\
\text { nitrogen (as nitrogen) }\end{array}$ & .2 & .4 & .3 & .3 & 3 & 3 \\
\hline $\begin{array}{l}\text { Nitrite plus nitrate } \\
\text { (as nitrogen) }\end{array}$ & 1.9 & 2.2 & 2.0 & 2.0 & 13 & 13 \\
\hline $\begin{array}{l}\text { Orthophosphate (as } \\
\text { phosphorus) }\end{array}$ & $<.01$ & .03 & .02 & .02 & 13 & 12 \\
\hline \multirow[t]{2}{*}{ Organic carbon } & .3 & 1.4 & .5 & .6 & 13 & 13 \\
\hline & \multicolumn{6}{|c|}{ NRF-6 } \\
\hline Ammonia (as nitrogen) & $<.01$ & .02 & .02 & .02 & 11 & 7 \\
\hline $\begin{array}{l}\text { Nitrite plus nitrate } \\
\text { (as nitrogen) }\end{array}$ & 1.6 & 1.9 & 1.7 & 1.7 & 11 & 11 \\
\hline $\begin{array}{l}\text { Orthophosphate (as } \\
\text { phosphorus) }\end{array}$ & .02 & .08 & .07 & .07 & 12 & 12 \\
\hline Organic carbon & .5 & 1.2 & .6 & .8 & 11 & 11 \\
\hline Phenols & 1 & 5 & 2 & 2 & 7 & 7 \\
\hline \multirow[t]{2}{*}{ Turbidity } & .3 & .7 & .5 & .5 & 7 & 7 \\
\hline & \multicolumn{6}{|c|}{ NRF-7 } \\
\hline Ammonia (as nitrogen) & $<.01$ & .05 & .02 & .02 & 12 & 7 \\
\hline $\begin{array}{l}\text { Nitrite plus nitrate } \\
\text { (as nitrogen) }\end{array}$ & .38 & 1.8 & .465 & .57 & 12 & 12 \\
\hline $\begin{array}{l}\text { Orthophosphate (as } \\
\text { phosphorus) }\end{array}$ & $<.01$ & .03 & .02 & .02 & 12 & 9 \\
\hline Organic carbon & .1 & .9 & .4 & .4 & 13 & 13 \\
\hline Phenols & $<1$ & 2 & 1 & 1 & 7 & 5 \\
\hline \multirow[t]{2}{*}{ Turbidity } & 1.7 & 7.3 & 3.6 & 3.6 & 7 & 7 \\
\hline & \multicolumn{6}{|c|}{ USGS 12} \\
\hline Ammonia (as nitrogen) & $<.01$ & .06 & .02 & .02 & 10 & 8 \\
\hline $\begin{array}{l}\text { Ammonia plus organic } \\
\text { nitrogen (as nitrogen) }\end{array}$ & $<.2$ & .3 & .25 & .2 & 3 & 2 \\
\hline Nitrite (as nitrogen) & $<.01$ & .02 & .015 & .02 & 13 & 2 \\
\hline $\begin{array}{l}\text { Nitrite plus nitrate } \\
\text { (as nitrogen) }\end{array}$ & .46 & 2.1 & 2.0 & 1.8 & 13 & 13 \\
\hline
\end{tabular}


Table 8. Statistical parameters for dissolved nutrients, total organic carbon, total phenols, and turbidity, by well-Continued

\begin{tabular}{|c|c|c|c|c|c|c|}
\hline \multirow{2}{*}{$\begin{array}{l}\text { Constituent or physical } \\
\text { characteristic }\end{array}$} & \multicolumn{6}{|c|}{ Statistical parameter } \\
\hline & Minimum & Maximum & Median & Mean & $\begin{array}{l}\text { Sample } \\
\text { size }\end{array}$ & $\begin{array}{l}\text { Mean and median } \\
\text { sample size }\end{array}$ \\
\hline & \multicolumn{6}{|c|}{ USGS 12 - cont. } \\
\hline $\begin{array}{l}\text { Orthophosphate (as } \\
\text { phosphorus) }\end{array}$ & $<.01$ & .04 & .02 & .02 & 13 & 11 \\
\hline \multirow[t]{2}{*}{ Organic carbon } & .3 & 1.7 & .4 & .5 & 13 & 13 \\
\hline & \multicolumn{6}{|c|}{ USGS 15} \\
\hline Ammonia (as nitrogen) & $<.01$ & .04 & .025 & .03 & 10 & 6 \\
\hline $\begin{array}{l}\text { Nitrite plus nitrate } \\
\text { (as nitrogen) }\end{array}$ & .30 & 1.3 & .545 & .67 & 12 & 12 \\
\hline $\begin{array}{l}\text { Orthophosphate (as } \\
\text { phosphorus) }\end{array}$ & $<.01$ & .04 & .02 & .02 & 12 & 10 \\
\hline \multirow[t]{2}{*}{ Organic carbon } & $<.1$ & 3.8 & .3 & .6 & 12 & 11 \\
\hline & \multicolumn{6}{|c|}{ USGS 17} \\
\hline Ammonia (as nitrogen) & $<.01$ & .04 & .02 & .02 & 10 & 6 \\
\hline Nitrite (as nitrogen) & $<.01$ & .02 & .02 & .02 & 14 & 2 \\
\hline $\begin{array}{l}\text { Nitrite plus nitrate } \\
\text { (as nitrogen) }\end{array}$ & .30 & .4 & .335 & .34 & 14 & 14 \\
\hline $\begin{array}{l}\text { Orthophosphate (as } \\
\text { phosphorus) }\end{array}$ & $<.01$ & .03 & .01 & .02 & 14 & 12 \\
\hline \multirow[t]{2}{*}{ Organic carbon } & $<.01$ & 1.0 & .2 & .3 & 14 & 13 \\
\hline & \multicolumn{6}{|c|}{ USGS 97} \\
\hline Ammonia (as nitrogen) & $<.01$ & .02 & .02 & .02 & 10 & 6 \\
\hline $\begin{array}{l}\text { Ammonia plus organic } \\
\text { nitrogen (as nitrogen) }\end{array}$ & $<.2$ & .3 & .2 & .2 & 4 & 3 \\
\hline Nitrite (as nitrogen) & $<.01$ & .02 & .015 & .02 & 14 & 2 \\
\hline $\begin{array}{l}\text { Nitrite plus nitrate } \\
\text { (as nitrogen) }\end{array}$ & 1.9 & 2.1 & 2.0 & 2.0 & 14 & 14 \\
\hline $\begin{array}{l}\text { Orthophosphate (as } \\
\text { phosphorus) }\end{array}$ & $<.01$ & .04 & .02 & .02 & 14 & 13 \\
\hline \multirow[t]{2}{*}{ Organic carbon } & .3 & .8 & .45 & .5 & 14 & 14 \\
\hline & \multicolumn{6}{|c|}{ USGS 98} \\
\hline Ammonia (as nitrogen) & $<.01$ & .04 & .02 & .02 & 8 & 5 \\
\hline $\begin{array}{l}\text { Nitrite plus nitrate } \\
\text { (as nitrogen) }\end{array}$ & 1.0 & 1.1 & 1.1 & 1.1 & 11 & 11 \\
\hline
\end{tabular}


Table 8.-Statistical parameters for dissolved nutrients, total organic carbon, total phenols, and turbidity, by well-Continued

\begin{tabular}{|c|c|c|c|c|c|c|}
\hline \multirow{2}{*}{$\begin{array}{l}\text { Constituent or physical } \\
\text { characteristic }\end{array}$} & \multicolumn{6}{|c|}{ Statistical parameter } \\
\hline & Minimum & Maximum & Median & Mean & $\begin{array}{l}\text { Sample } \\
\text { size }\end{array}$ & $\begin{array}{l}\text { Mean and median } \\
\text { sample size }\end{array}$ \\
\hline & \multicolumn{6}{|c|}{ USGS 98 - cont. } \\
\hline $\begin{array}{l}\text { Orthophosphate (as } \\
\text { phosphorus) }\end{array}$ & $<.01$ & .02 & .02 & .02 & 11 & 9 \\
\hline \multirow[t]{2}{*}{ Organic carbon } & $<.1$ & 1 & .25 & .4 & 11 & 10 \\
\hline & \multicolumn{6}{|c|}{ USGS 99} \\
\hline Ammonia (as nitrogen) & $<.01$ & .03 & .015 & .02 & 10 & 6 \\
\hline Nitrite (as nitrogen) & $<.01$ & .02 & .015 & .02 & 13 & 2 \\
\hline $\begin{array}{l}\text { Nitrite plus nitrate } \\
\text { (as nitrogen) }\end{array}$ & 1.5 & 1.6 & 1.5 & 1.5 & 13 & 13 \\
\hline $\begin{array}{l}\text { Orthophosphate (as } \\
\text { phosphorus) }\end{array}$ & $<.01$ & .02 & .02 & .02 & 13 & 11 \\
\hline \multirow[t]{2}{*}{ Organic carbon } & .1 & 1.7 & .4 & .5 & 13 & 13 \\
\hline & \multicolumn{6}{|c|}{ USGS 102} \\
\hline Ammonia (as nitrogen) & $<.01$ & .02 & .02 & .02 & 10 & 5 \\
\hline Nitrite (as nitrogen) & $<.01$ & .02 & .02 & .02 & 12 & 2 \\
\hline $\begin{array}{l}\text { Nitrite plus nitrate } \\
\text { (as nitrogen) }\end{array}$ & 1.7 & 2 & 1.8 & 1.8 & 12 & 12 \\
\hline $\begin{array}{l}\text { Orthophosphate (as } \\
\text { phosphorus) }\end{array}$ & $<.01$ & .03 & .02 & .02 & 12 & 11 \\
\hline \multirow[t]{2}{*}{ Organic carbon } & .2 & .8 & .4 & .4 & 12 & 12 \\
\hline & \multicolumn{6}{|c|}{ Water Supply INEL-1 } \\
\hline Ammonia (as nitrogen) & $<.01$ & .04 & .02 & .02 & 10 & 6 \\
\hline $\begin{array}{l}\text { Ammonia plus organic } \\
\text { nitrogen (as nitrogen) }\end{array}$ & .4 & .5 & .4 & .4 & 3 & 3 \\
\hline Nitrite (as nitrogen) & $<.01$ & .02 & .015 & .02 & 13 & 2 \\
\hline $\begin{array}{l}\text { Nitrite plus nitrate } \\
\text { (as nitrogen) }\end{array}$ & 4.5 & 5.4 & 5.0 & 4.9 & 13 & 13 \\
\hline $\begin{array}{l}\text { Orthophosphate (as } \\
\text { phosphorus) }\end{array}$ & $<.01$ & .02 & .01 & .01 & 13 & 10 \\
\hline Organic carbon & .5 & 1.2 & .8 & .8 & 13 & 13 \\
\hline
\end{tabular}


Table 9.-Concentrations of gross alpha-particle radioactivity in water from round-three samples, Naval Reactors Facility and vicinity

[Analyses were performed by the U.S. Geological Survey's National Water Quality Laboratory using a residue procedure. Raw field samples were processed in laboratory prior to analysis. Analytical results and uncertainties - for example, $\mathbf{2 . 7 7} \pm \mathbf{0 . 4 4 4}$ in indicated units. Analytical uncertainties are reported as $1 \mathrm{~s}$. Concentrations that meet or exceed the reporting level of 3 times the $1 \mathrm{~s}$ value are shown in boldface type. Abbreviations: (m/d/y), month/day/year; $\mu \mathrm{g} / \mathrm{L}$, microgram per liter; $\mathrm{pCi} / \mathrm{L}$, picocurie per liter. Symbol: NR indicates analysis not requested. Sample identifier: see figure 2 for location of wells; QAS indicates quality-assurance samples, 23 and 30 are blank samples, others are replicates]

\begin{tabular}{|c|c|c|c|c|c|}
\hline \multirow[b]{2}{*}{$\begin{array}{l}\text { Sample } \\
\text { identifier }\end{array}$} & \multirow{2}{*}{$\begin{array}{c}\text { Date } \\
\text { sampled } \\
(\mathrm{m} / \mathrm{d} / \mathrm{y})\end{array}$} & \multicolumn{2}{|c|}{ Dissolved } & \multicolumn{2}{|c|}{ Suspended } \\
\hline & & $\begin{array}{l}\text { as uranium } \\
(\mu \mathrm{g} / \mathrm{L})\end{array}$ & $\begin{array}{l}\text { as thorium-230 } \\
\qquad(\mathrm{pCi} / \mathrm{L})\end{array}$ & $\begin{array}{l}\text { as uranium } \\
(\mu \mathrm{g} / \mathrm{L})\end{array}$ & $\begin{array}{l}\text { as thorium-230 } \\
\qquad(\mathrm{pCi} / \mathrm{L})\end{array}$ \\
\hline QAS-23 & $6 / 12 / 92$ & $0.231 \pm 0.164$ & $0.157 \pm 0.111$ & $0.019 \pm 0.166$ & $0.010 \pm .090$ \\
\hline QAS-30 & $6 / 15 / 93$ & $-.018 \pm 0.060$ & $-.011 \pm 0.036$ & NR & NR \\
\hline \multirow[t]{3}{*}{ NRF-1 } & $3 / 5 / 91$ & $2.77 \pm 0.444$ & $2.50 \pm 0.422$ & $.132 \pm 0.145$ & $.073 \pm 0.083$ \\
\hline & $6 / 17 / 91$ & $4.01 \pm 0.60$ & $2.78 \pm 0.423$ & $-.111 \pm 0.079$ & $-.061 \pm 0.045$ \\
\hline & 9/9/91 & $3.33 \pm 0.56$ & $2.33 \pm 0.392$ & $-.119 \pm 0.116$ & $-.064 \pm 0.062$ \\
\hline QAS-18 & 9/9/91 & $3.12 \pm 0.52$ & $2.19 \pm 0.368$ & $-.028 \pm 0.148$ & $-.016 \pm 0.084$ \\
\hline \multirow[t]{9}{*}{ NRF-1 } & $12 / 4 / 91$ & $3.92 \pm 0.60$ & $2.80 \pm 0.428$ & $.029 \pm 0.256$ & $.016 \pm 0.137$ \\
\hline & $3 / 11 / 92$ & $1.77 \pm 0.394$ & $1.23 \pm 0.276$ & $.028 \pm 0.138$ & $.015 \pm 0.076$ \\
\hline & $6 / 11 / 92$ & $2.45 \pm 0.474$ & $1.69 \pm 0.328$ & $.606 \pm 0.316$ & $.333 \pm 0.186$ \\
\hline & $9 / 16 / 92$ & $2.67 \pm 0.488$ & $1.87 \pm 0.344$ & $.075 \pm 0.154$ & $.041 \pm 0.084$ \\
\hline & $12 / 3 / 92$ & $6.37 \pm 1.36$ & $4.73 \pm 1.01$ & $.636 \pm 0.346$ & $.370 \pm 0.204$ \\
\hline & $4 / 7 / 93$ & $4.14 \pm 0.76$ & $2.99 \pm 0.54$ & $.004 \pm 0.096$ & $.002 \pm 0.053$ \\
\hline & $6 / 15 / 93$ & $3.83 \pm 1.15$ & $2.72 \pm 0.82$ & NR & NR \\
\hline & $9 / 15 / 93$ & $6.76 \pm 1.52$ & $5.01 \pm 1.12$ & NR & NR \\
\hline & $11 / 4 / 93$ & $5.42 \pm 1.36$ & $3.91 \pm 0.98$ & NR & NR \\
\hline NRF-2 & $3 / 5 / 91$ & $2.81+0.442$ & $2.84 \pm 0.447$ & $.539 \pm 0.298$ & $.291 \pm 0.164$ \\
\hline QAS-13 & $3 / 5 / 91$ & $3.01+0.436$ & $3.02 \pm 0.440$ & $-.122 \pm 0.118$ & $-.066 \pm 0.064$ \\
\hline \multirow[t]{5}{*}{ NRF-2 } & $6 / 17 / 91$ & $3.85 \pm 0.60$ & $2.66 \pm 0.424$ & $-.085 \pm 0.162$ & $-.048 \pm 0.092$ \\
\hline & 9/9/91 & $3.50 \pm 0.56$ & $2.43 \pm 0.392$ & $.113 \pm 0.191$ & $.060 \pm 0.102$ \\
\hline & $12 / 4 / 91$ & $3.56 \pm 0.57$ & $2.48 \pm 0.400$ & $-.162 \pm 0.214$ & $-.086 \pm 0.115$ \\
\hline & $3 / 11 / 92$ & $4.64 \pm 0.70$ & $2.90 \pm 0.464$ & $-.083 \pm 0.158$ & $-.044 \pm 0.084$ \\
\hline & $6 / 11 / 92$ & $2.28 \pm 0.450$ & $1.58 \pm 0.314$ & $-.274 \pm 0.124$ & $-.158 \pm 0.077$ \\
\hline
\end{tabular}


Table 9.-Concentrations of gross alpha-particle radioactivity in water from round-three samples, Naval Reactors Facility and vicinity-Continued

\begin{tabular}{|c|c|c|c|c|c|}
\hline \multirow[b]{2}{*}{$\begin{array}{l}\text { Sample } \\
\text { identifier }\end{array}$} & \multirow{2}{*}{$\begin{array}{c}\text { Date } \\
\text { sampled } \\
(\mathrm{m} / \mathrm{d} / \mathrm{y})\end{array}$} & \multicolumn{2}{|c|}{ Dissolved } & \multicolumn{2}{|c|}{ Suspended } \\
\hline & & $\begin{array}{l}\text { as uranium } \\
(\mu \mathrm{g} / \mathrm{L})\end{array}$ & $\begin{array}{l}\text { as thorium-230 } \\
\qquad(\mathrm{pCi} / \mathrm{L})\end{array}$ & $\begin{array}{l}\text { as uranium } \\
(\mu \mathrm{g} / \mathrm{L})\end{array}$ & $\begin{array}{l}\text { as thorium-230 } \\
\qquad(\mathrm{pCi} / \mathrm{L})\end{array}$ \\
\hline \multirow[t]{5}{*}{ NRF-2 } & $9 / 16 / 92$ & $3.69 \pm 0.58$ & $2.63 \pm 0.412$ & $.019 \pm 0.164$ & $.010 \pm 0.089$ \\
\hline & $12 / 3 / 92$ & $5.49 \pm 1.36$ & $3.97 \pm 0.98$ & $-.046 \pm 0.191$ & $-.024 \pm 0.101$ \\
\hline & $4 / 7 / 93$ & $4.53 \pm 0.82$ & $2.82 \pm 0.53$ & $-.067 \pm 0.028$ & $-.036 \pm 0.016$ \\
\hline & $6 / 15 / 93$ & $3.97 \pm 1.18$ & $3.06 \pm 0.90$ & NR & NR \\
\hline & $9 / 15 / 93$ & $4.56 \pm 1.30$ & $3.38 \pm 0.96$ & NR & NR \\
\hline QAS-31 & $9 / 15 / 93$ & $5.73 \pm 1.50$ & $4.42 \pm 1.16$ & NR & NR \\
\hline NRF-2 & $11 / 4 / 93$ & $4.68 \pm 1.40$ & $3.38 \pm 1.00$ & NR & NR \\
\hline NRF-3 & $6 / 17 / 91$ & $4.38 \pm 0.64$ & $3.04 \pm 0.436$ & $.083 \pm 0.126$ & $.047 \pm 0.072$ \\
\hline QAS-16 & $6 / 17 / 91$ & $3.66 \pm 0.60$ & $2.26 \pm 0.390$ & $-.028 \pm 0.152$ & $-.015 \pm 0.082$ \\
\hline \multirow[t]{6}{*}{ NRF-3 } & $9 / 26 / 91$ & $3.21 \pm 0.56$ & $1.98 \pm 0.365$ & $-.028 \pm 0.148$ & $-.016 \pm 0.084$ \\
\hline & $12 / 4 / 91$ & $3.24 \pm 0.57$ & $2.01 \pm 0.372$ & $.065 \pm 0.176$ & $.036 \pm 0.097$ \\
\hline & $3 / 11 / 92$ & $4.00 \pm 0.63$ & $2.81 \pm 0.442$ & $.159 \pm 0.200$ & $.086 \pm 0.110$ \\
\hline & $6 / 11 / 92$ & $1.53 \pm 0.345$ & $1.01 \pm 0.228$ & $-.092 \pm 0.178$ & $-.053 \pm 0.103$ \\
\hline & $9 / 16 / 92$ & $3.02 \pm 0.52$ & $2.11 \pm 0.370$ & $6.58 \pm 1.84$ & $5.08 \pm 1.60$ \\
\hline & $12 / 3 / 92$ & $5.13 \pm 1.22$ & $3.69 \pm 0.88$ & $-.348 \pm 0.164$ & $-.200 \pm 0.096$ \\
\hline QAS-26 & $12 / 3 / 92$ & $5.12 \pm 1.42$ & $3.95 \pm 1.09$ & $.122 \pm 0.168$ & $.066 \pm 0.092$ \\
\hline \multirow[t]{4}{*}{ NRF-3 } & $4 / 8 / 93$ & $3.38 \pm 0.72$ & $2.12 \pm 0.461$ & $.090 \pm 0.170$ & $.049 \pm 0.092$ \\
\hline & $6 / 15 / 93$ & $6.17 \pm 1.48$ & $3.95 \pm 0.96$ & NR & NR \\
\hline & $9 / 15 / 93$ & $4.96 \pm 1.37$ & $3.82 \pm 1.06$ & NR & NR \\
\hline & $11 / 4 / 93$ & $3.47 \pm 1.04$ & $2.44 \pm 0.73$ & NR & NR \\
\hline \multirow[t]{8}{*}{ NRF-4 } & $3 / 5 / 91$ & $2.89 \pm 0.428$ & $2.87 \pm 0.432$ & $.074 \pm 0.152$ & $.041 \pm 0.086$ \\
\hline & $6 / 17 / 91$ & $3.78 \pm 0.60$ & $2.69 \pm 0.424$ & $.401 \pm 0.212$ & $.218 \pm 0.120$ \\
\hline & 9/9/91 & $3.77 \pm 0.58$ & $2.66 \pm 0.410$ & $.066 \pm 0.179$ & $.036 \pm 0.098$ \\
\hline & $12 / 4 / 91$ & $3.80 \pm 0.60$ & $2.72 \pm 0.426$ & $-.067 \pm 0.236$ & $-.036 \pm 0.128$ \\
\hline & $3 / 11 / 92$ & $3.40 \pm 0.56$ & $2.37 \pm 0.388$ & $.029 \pm 0.145$ & $.015 \pm 0.076$ \\
\hline & $6 / 11 / 92$ & $1.60 \pm 0.382$ & $.972 \pm 0.240$ & $-.345 \pm 0.180$ & $-.190 \pm 0.103$ \\
\hline & $9 / 16 / 92$ & $4.37 \pm 0.66$ & $2.71 \pm 0.438$ & $.009 \pm 0.183$ & $.005 \pm 0.096$ \\
\hline & $12 / 3 / 92$ & $4.40 \pm 1.14$ & $3.27 \pm 0.84$ & $.056 \pm 0.198$ & $.030 \pm 0.107$ \\
\hline
\end{tabular}


Table 9.-Concentrations of gross alpha-particle radioactivity in water from round-three samples, Naval Reactors Facility and vicinity-Continued

\begin{tabular}{|c|c|c|c|c|c|}
\hline \multirow[b]{2}{*}{$\begin{array}{l}\text { Sample } \\
\text { identifier }\end{array}$} & \multirow{2}{*}{$\begin{array}{c}\text { Date } \\
\text { sampled } \\
(\mathrm{m} / \mathrm{d} / \mathrm{y})\end{array}$} & \multicolumn{2}{|c|}{ Dissolved } & \multicolumn{2}{|c|}{ Suspended } \\
\hline & & $\begin{array}{l}\text { as uranium } \\
\qquad(\mu \mathrm{g} / \mathrm{L})\end{array}$ & $\begin{array}{l}\text { as thorium-230 } \\
\qquad(\mathrm{pCi} / \mathrm{L})\end{array}$ & $\begin{array}{l}\text { as uranium } \\
(\mu \mathrm{g} / \mathrm{L})\end{array}$ & $\begin{array}{l}\text { as thorium-230 } \\
\qquad(\mathrm{pCi} / \mathrm{L})\end{array}$ \\
\hline NRF-4 & $4 / 7 / 93$ & $3.56 \pm 0.61$ & $2.49 \pm 0.431$ & $.120 \pm 0.136$ & $.065 \pm 0.074$ \\
\hline QAS-28 & $4 / 7 / 93$ & $3.03 \pm 0.62$ & $2.10 \pm 0.436$ & $.217 \pm 0.194$ & $.116 \pm 0.106$ \\
\hline \multirow[t]{3}{*}{ NRF-4 } & $6 / 15 / 93$ & $4.75 \pm 1.42$ & $3.04 \pm 0.92$ & NR & NR \\
\hline & $9 / 15 / 93$ & $6.48 \pm 1.70$ & $4.11 \pm 1.08$ & NR & NR \\
\hline & $11 / 4 / 93$ & $1.35 \pm 0.68$ & $.996 \pm 0.50$ & NR & NR \\
\hline \multirow[t]{5}{*}{ NRF-6 } & $9 / 18 / 92$ & $5.08 \pm 0.70$ & $3.53 \pm 0.487$ & $.068 \pm 0.184$ & $.036 \pm 0.098$ \\
\hline & $12 / 9 / 92$ & $2.88 \pm 1.72$ & $2.13 \pm 1.28$ & $-.091 \pm 0.178$ & $-.049 \pm 0.096$ \\
\hline & $4 / 9 / 93$ & $3.45 \pm 0.94$ & $2.38 \pm 0.64$ & $.111 \pm 0.121$ & $.058 \pm 0.064$ \\
\hline & $9 / 14 / 93$ & $5.92 \pm 2.62$ & $4.20 \pm 1.86$ & NR & NR \\
\hline & $11 / 4 / 93$ & $3.63 \pm 2.16$ & $2.80 \pm 1.66$ & NR & NR \\
\hline \multirow[t]{6}{*}{ NRF-7 } & $9 / 18 / 92$ & $3.37 \pm 0.54$ & $2.32 \pm 0.368$ & $.064 \pm 0.174$ & $.037 \pm 0.099$ \\
\hline & $12 / 9 / 92$ & $2.55 \pm 0.63$ & $1.84 \pm 0.452$ & $-.134 \pm 0.162$ & $-.082 \pm 0.101$ \\
\hline & 4/9/93 & $1.91 \pm 0.426$ & $1.33 \pm 0.298$ & $.225 \pm 0.190$ & $.130 \pm 0.114$ \\
\hline & $6 / 10 / 93$ & $2.69 \pm 0.68$ & $1.99 \pm 0.50$ & NR & NR \\
\hline & $9 / 14 / 93$ & $1.46 \pm 0.58$ & $.935 \pm 0.370$ & NR & NR \\
\hline & $11 / 3 / 93$ & $1.92 \pm 0.58$ & $1.37 \pm 0.416$ & NR & NR \\
\hline \multirow[t]{2}{*}{ USGS 12} & $6 / 10 / 91$ & $4.26 \pm 0.62$ & $2.95 \pm 0.436$ & $.086 \pm 0.130$ & $.045 \pm 0.070$ \\
\hline & $9 / 6 / 91$ & $3.16 \pm 0.55$ & $2.23 \pm 0.389$ & $.034 \pm 0.099$ & $.022 \pm 0.065$ \\
\hline QAS-17 & $9 / 6 / 91$ & $3.03 \pm 0.54$ & $2.09 \pm 0.372$ & $.175 \pm 0.156$ & $.098 \pm 0.090$ \\
\hline \multirow[t]{9}{*}{ USGS 12} & $12 / 5 / 91$ & $4.21 \pm 0.62$ & $2.89 \pm 0.434$ & $-.212 \pm 0.214$ & $-.112 \pm 0.114$ \\
\hline & $3 / 12 / 92$ & $3.46 \pm 0.58$ & $2.41 \pm 0.410$ & $.373 \pm 0.212$ & $.197 \pm 0.117$ \\
\hline & $6 / 19 / 92$ & $3.33 \pm 0.54$ & $2.30 \pm 0.376$ & $.028 \pm 0.140$ & $.015 \pm 0.076$ \\
\hline & $9 / 18 / 92$ & $1.51 \pm 0.390$ & $1.05 \pm 0.272$ & $.163 \pm 0.175$ & $.097 \pm 0.106$ \\
\hline & $12 / 1 / 92$ & $1.84 \pm 0.57$ & $1.17 \pm 0.362$ & $3.75 \pm 1.06$ & $3.72 \pm 1.06$ \\
\hline & $4 / 13 / 93$ & $3.28 \pm 0.62$ & $2.29 \pm 0.436$ & $-.018 \pm 0.120$ & $-.010 \pm 0.064$ \\
\hline & $6 / 14 / 93$ & $4.48 \pm 1.13$ & $3.24 \pm 0.82$ & NR & NR \\
\hline & $9 / 16 / 93$ & $5.38 \pm 1.39$ & $3.85 \pm 1.00$ & NR & NR \\
\hline & $11 / 5 / 93$ & $4.50 \pm 1.24$ & $3.33 \pm 0.92$ & NR & NR \\
\hline
\end{tabular}


Table 9.-Concentrations of gross alpha-particle radioactivity in water from round-three samples, Naval Reactors Facility and vicinity-Continued

\begin{tabular}{|c|c|c|c|c|c|}
\hline \multirow[b]{2}{*}{$\begin{array}{l}\text { Sample } \\
\text { identifier }\end{array}$} & \multirow{2}{*}{$\begin{array}{c}\text { Date } \\
\text { sampled } \\
(\mathrm{m} / \mathrm{d} / \mathrm{y})\end{array}$} & \multicolumn{2}{|c|}{ Dissolved } & \multicolumn{2}{|c|}{ Suspended } \\
\hline & & $\begin{array}{l}\text { as uranium } \\
(\mu \mathrm{g} / \mathrm{L})\end{array}$ & $\begin{array}{l}\text { as thorium-230 } \\
\qquad(\mathrm{pCi} / \mathrm{L})\end{array}$ & $\begin{array}{l}\text { as uranium } \\
\qquad(\mu \mathrm{g} / \mathrm{L})\end{array}$ & $\begin{array}{l}\text { as thorium-230 } \\
\qquad(\mathrm{pCi} / \mathrm{L})\end{array}$ \\
\hline QAS-33 & $11 / 5 / 93$ & $5.15 \pm 1.26$ & $3.59 \pm 0.88$ & NR & NR \\
\hline \multirow[t]{4}{*}{ USGS 15} & $6 / 10 / 91$ & $3.72 \pm 0.58$ & $2.56 \pm 0.410$ & $-.076 \pm 0.136$ & $-.040 \pm .073$ \\
\hline & $9 / 6 / 91$ & $2.33 \pm 0.490$ & $1.45 \pm 0.315$ & $.028 \pm 0.251$ & $.016 \pm .142$ \\
\hline & $12 / 4 / 91$ & $2.15 \pm 0.431$ & $1.47 \pm 0.294$ & $-.236 \pm 0.144$ & $-.126 \pm 0.080$ \\
\hline & $3 / 12 / 92$ & $1.87 \pm 0.426$ & $1.37 \pm 0.312$ & $.066 \pm 0.178$ & $.035 \pm 0.096$ \\
\hline QAS-22 & $3 / 12 / 92$ & $2.48 \pm 0.474$ & $1.74 \pm 0.334$ & $-.009 \pm 0.087$ & $-.005 \pm 0.048$ \\
\hline \multirow[t]{7}{*}{ USGS 15} & $6 / 19 / 92$ & $1.90 \pm 0.395$ & $1.29 \pm 0.267$ & $29.0 \pm 14.9$ & $22.4 \pm 11.45$ \\
\hline & $9 / 17 / 92$ & $1.62 \pm 0.380$ & $1.12 \pm 0.263$ & $.342 \pm 0.238$ & $.205 \pm 0.144$ \\
\hline & $12 / 1 / 92$ & $3.96 \pm 1.24$ & $3.06 \pm 0.95$ & $.499 \pm 0.254$ & $.274 \pm 0.148$ \\
\hline & $4 / 13 / 93$ & $2.04 \pm 0.461$ & $1.28 \pm 0.296$ & $-.066 \pm 0.104$ & $-.035 \pm 0.056$ \\
\hline & $6 / 14 / 93$ & $3.71 \pm 0.86$ & $2.85 \pm 0.66$ & NR & NR \\
\hline & $9 / 16 / 93$ & $3.54 \pm 0.96$ & $2.56 \pm 0.69$ & NR & NR \\
\hline & $11 / 5 / 93$ & $2.24 \pm 0.73$ & $1.72 \pm 0.56$ & NR & NR \\
\hline USGS 17 & $3 / 13 / 91$ & $1.26 \pm 0.292$ & $1.26 \pm 0.293$ & $.107 \pm 0.216$ & $.056 \pm 0.114$ \\
\hline QAS-14 & $3 / 13 / 91$ & $1.51 \pm 0.318$ & $1.35 \pm 0.296$ & $.365 \pm 0.208$ & $.197 \pm 0.117$ \\
\hline \multirow[t]{9}{*}{ USGS 17} & $6 / 6 / 91$ & $2.56 \pm 0.492$ & $1.83 \pm 0.352$ & $.215 \pm 0.192$ & $.117 \pm 0.106$ \\
\hline & $9 / 5 / 91$ & $2.21 \pm 0.454$ & $1.58 \pm 0.325$ & $-.121 \pm 0.118$ & $-.067 \pm 0.066$ \\
\hline & $12 / 3 / 91$ & $2.06 \pm 0.432$ & $1.42 \pm 0.301$ & $.061 \pm 0.254$ & $.037 \pm 0.153$ \\
\hline & $3 / 16 / 92$ & $2.07 \pm 0.434$ & $1.42 \pm 0.300$ & $.216 \pm 0.166$ & $.131 \pm 0.104$ \\
\hline & $6 / 11 / 92$ & $1.51 \pm 0.386$ & $.931 \pm 0.244$ & $.064 \pm 0.172$ & $.040 \pm 0.108$ \\
\hline & $9 / 16 / 92$ & $4.11 \pm 0.62$ & $2.94 \pm 0.443$ & $-.292 \pm 0.154$ & $-.157 \pm 0.088$ \\
\hline & $12 / 3 / 92$ & $3.14 \pm 0.74$ & $2.00 \pm 0.472$ & $.046 \pm 0.210$ & $.026 \pm 0.122$ \\
\hline & $4 / 7 / 93$ & $2.02 \pm 0.420$ & $1.39 \pm 0.289$ & $-.029 \pm 0.154$ & $-.015 \pm 0.081$ \\
\hline & $6 / 11 / 93$ & $1.91 \pm 0.58$ & $1.38 \pm 0.414$ & NR & NR \\
\hline QAS-29 & $6 / 11 / 93$ & $2.81 \pm 0.72$ & $2.08 \pm 0.53$ & NR & NR \\
\hline \multirow[t]{2}{*}{ USGS 17} & $9 / 15 / 93$ & $2.04 \pm 0.68$ & $1.45 \pm 0.480$ & NR & NR \\
\hline & $11 / 8 / 93$ & $2.31 \pm 0.68$ & $1.66 \pm 0.488$ & NR & NR \\
\hline USGS 97 & $3 / 13 / 91$ & $3.18 \pm 0.453$ & $3.21 \pm 0.461$ & $.280 \pm 0.284$ & $.150 \pm 0.153$ \\
\hline
\end{tabular}


Table 9.-Concentrations of gross alpha-particle radioactivity in water from round-three samples, Naval Reactors Facility and vicinity-Continued

\begin{tabular}{|c|c|c|c|c|c|}
\hline \multirow[b]{2}{*}{$\begin{array}{l}\text { Sample } \\
\text { identifier }\end{array}$} & \multirow{2}{*}{$\begin{array}{c}\text { Date } \\
\text { sampled } \\
(\mathrm{m} / \mathrm{d} / \mathrm{y})\end{array}$} & \multicolumn{2}{|c|}{ Dissolved } & \multicolumn{2}{|c|}{ Suspended } \\
\hline & & $\begin{array}{l}\text { as uranium } \\
(\mu \mathrm{g} / \mathrm{L})\end{array}$ & $\begin{array}{l}\text { as thorium-230 } \\
\qquad(\mathrm{pCi} / \mathrm{L})\end{array}$ & $\begin{array}{l}\text { as uranium } \\
(\mu \mathrm{g} / \mathrm{L})\end{array}$ & $\begin{array}{l}\text { as thorium-230 } \\
\qquad(\mathrm{pCi} / \mathrm{L})\end{array}$ \\
\hline USGS 97 & $6 / 7 / 91$ & $4.96 \pm 0.68$ & $3.47 \pm 0.482$ & $.185 \pm 0.165$ & $.097 \pm 0.087$ \\
\hline QAS-15 & $6 / 7 / 91$ & $4.50 \pm 0.64$ & $3.13 \pm 0.453$ & $.039 \pm 0.114$ & $.020 \pm 0.060$ \\
\hline \multirow[t]{10}{*}{ USGS 97} & $9 / 5 / 91$ & $3.78 \pm 0.58$ & $2.60 \pm 0.396$ & $.018 \pm 0.159$ & $.011 \pm 0.094$ \\
\hline & $12 / 3 / 91$ & $3.67 \pm 0.58$ & $2.55 \pm 0.403$ & $-.305 \pm 0.180$ & $-.162 \pm 0.100$ \\
\hline & $3 / 16 / 92$ & $2.74 \pm 0.484$ & $1.87 \pm 0.330$ & $.009 \pm 0.183$ & $.005 \pm 0.096$ \\
\hline & $6 / 17 / 92$ & $2.15 \pm 0.469$ & $1.34 \pm 0.302$ & $-.028 \pm 0.152$ & $-.015 \pm 0.083$ \\
\hline & $9 / 21 / 92$ & $3.23 \pm 0.54$ & $2.26 \pm 0.383$ & $.212 \pm 0.190$ & $.112 \pm 0.102$ \\
\hline & $12 / 8 / 92$ & $2.58 \pm 0.78$ & $1.86 \pm 0.56$ & $.122 \pm 0.168$ & $.068 \pm 0.094$ \\
\hline & $4 / 6 / 93$ & $3.20 \pm 0.74$ & $2.33 \pm 0.54$ & $-.030 \pm 0.100$ & $-.016 \pm 0.054$ \\
\hline & $6 / 9 / 93$ & $5.93 \pm 1.41$ & $4.19 \pm 1.00$ & NR & NR \\
\hline & $9 / 13 / 93$ & $5.93 \pm 1.30$ & $4.17 \pm 0.92$ & NR & NR \\
\hline & $11 / 4 / 93$ & $2.29 \pm 0.88$ & $1.70 \pm 0.66$ & NR & NR \\
\hline QAS-32 & $11 / 4 / 93$ & $5.31 \pm 1.34$ & $3.79 \pm 0.96$ & NR & NR \\
\hline \multirow[t]{7}{*}{ USGS 98} & $3 / 13 / 91$ & $1.98 \pm 0.360$ & $1.76 \pm 0.336$ & $.179 \pm 0.160$ & $.096 \pm 0.088$ \\
\hline & $6 / 7 / 91$ & $2.97 \pm 0.54$ & $1.83 \pm 0.350$ & $.131 \pm 0.144$ & $.071 \pm 0.078$ \\
\hline & $9 / 5 / 91$ & $1.93 \pm 0.448$ & $1.20 \pm 0.286$ & $-.009 \pm 0.085$ & $-.005 \pm 0.051$ \\
\hline & $12 / 3 / 91$ & $2.03 \pm 0.427$ & $1.40 \pm 0.294$ & $.205 \pm 0.184$ & $.126 \pm 0.116$ \\
\hline & $3 / 16 / 92$ & $3.04 \pm 0.54$ & $2.12 \pm 0.377$ & $.127 \pm 0.175$ & $.066 \pm 0.092$ \\
\hline & $6 / 16 / 92$ & $2.45 \pm 0.488$ & $1.70 \pm 0.342$ & $-.046 \pm 0.192$ & $-.026 \pm 0.106$ \\
\hline & $9 / 21 / 92$ & $1.49 \pm 0.356$ & $1.02 \pm 0.242$ & $.107 \pm 0.216$ & $.056 \pm 0.114$ \\
\hline QAS-25 & $9 / 21 / 92$ & $2.97 \pm 0.52$ & $2.13 \pm 0.377$ & $.255 \pm 0.224$ & $.136 \pm 0.122$ \\
\hline \multirow[t]{3}{*}{ USGS 98} & $12 / 8 / 92$ & $3.27 \pm 0.84$ & $2.43 \pm 0.62$ & $.121 \pm 0.168$ & $.066 \pm 0.092$ \\
\hline & $4 / 6 / 93$ & $2.12 \pm 0.450$ & $1.52 \pm 0.324$ & $-.028 \pm 0.150$ & $-.015 \pm 0.082$ \\
\hline & $6 / 8 / 93$ & $3.44 \pm 0.96$ & $2.20 \pm 0.62$ & NR & NR \\
\hline \multirow[t]{4}{*}{ USGS 99} & $3 / 13 / 91$ & $2.22 \pm 0.394$ & $2.29 \pm 0.407$ & $.169 \pm 0.150$ & $.106 \pm 0.096$ \\
\hline & $6 / 7 / 91$ & $3.58 \pm 0.60$ & $2.21 \pm 0.392$ & $.084 \pm 0.128$ & $.045 \pm 0.070$ \\
\hline & $9 / 5 / 91$ & $2.85 \pm 0.50$ & $1.96 \pm 0.350$ & $.222 \pm 0.199$ & $.119 \pm 0.107$ \\
\hline & $12 / 3 / 91$ & $3.23 \pm 0.55$ & $2.25 \pm 0.382$ & $-.065 \pm 0.102$ & $-.036 \pm 0.057$ \\
\hline
\end{tabular}


Table 9.-Concentrations of gross alpha-particle radioactivity in water from round-three samples, Naval Reactors Facility and vicinity-Continued

\begin{tabular}{|c|c|c|c|c|c|}
\hline \multirow[b]{2}{*}{$\begin{array}{l}\text { Sample } \\
\text { identifier }\end{array}$} & \multirow{2}{*}{$\begin{array}{c}\text { Date } \\
\text { sampled } \\
(\mathrm{m} / \mathrm{d} / \mathrm{y})\end{array}$} & \multicolumn{2}{|c|}{ Dissolved } & \multicolumn{2}{|c|}{ Suspended } \\
\hline & & $\begin{array}{l}\text { as uranium } \\
(\mu \mathrm{g} / \mathrm{L})\end{array}$ & $\begin{array}{l}\text { as thorium-230 } \\
\qquad(\mathrm{pCi} / \mathrm{L})\end{array}$ & $\begin{array}{l}\text { as uranium } \\
(\mu \mathrm{g} / \mathrm{L})\end{array}$ & $\begin{array}{l}\text { as thorium-230 } \\
\qquad(\mathrm{pCi} / \mathrm{L})\end{array}$ \\
\hline \multirow[t]{2}{*}{ USGS 99} & $3 / 16 / 92$ & $2.87 \pm 0.50$ & $1.99 \pm 0.349$ & $-.028 \pm 0.152$ & $-.015 \pm 0.081$ \\
\hline & $6 / 16 / 92$ & $1.82 \pm 0.403$ & $1.27 \pm 0.280$ & $-.185 \pm 0.155$ & $-.103 \pm 0.088$ \\
\hline QAS-24 & $6 / 16 / 92$ & $1.76 \pm 0.419$ & $1.28 \pm 0.304$ & $.418 \pm 0.284$ & $.232 \pm 0.164$ \\
\hline \multirow[t]{6}{*}{ USGS 99} & $9 / 21 / 92$ & $2.43 \pm 0.478$ & $1.69 \pm 0.334$ & $-.105 \pm 0.206$ & $-.055 \pm 0.110$ \\
\hline & $12 / 8 / 92$ & $5.08 \pm 1.22$ & $3.24 \pm 0.78$ & $-.230 \pm 0.140$ & $-.122 \pm 0.076$ \\
\hline & $4 / 6 / 93$ & $3.07 \pm 0.56$ & $2.15 \pm 0.398$ & $.029 \pm 0.145$ & $.015 \pm 0.076$ \\
\hline & $6 / 9 / 93$ & $2.91 \pm 0.94$ & $2.08 \pm 0.68$ & NR & NR \\
\hline & $9 / 13 / 93$ & $3.80 \pm 1.10$ & $2.43 \pm 0.71$ & NR & NR \\
\hline & $11 / 2 / 93$ & $4.47 \pm 1.20$ & $2.85 \pm 0.77$ & NR & NR \\
\hline \multirow[t]{7}{*}{ USGS 102} & $6 / 7 / 91$ & $5.73 \pm 0.74$ & $4.11 \pm 0.54$ & $-.019 \pm 0.123$ & $-.010 \pm 0.067$ \\
\hline & $9 / 5 / 91$ & $3.16 \pm 0.56$ & $2.20 \pm 0.392$ & $.164 \pm 0.176$ & $.096 \pm 0.104$ \\
\hline & $12 / 3 / 91$ & $4.48 \pm 0.64$ & $3.10 \pm 0.436$ & $-.092 \pm 0.180$ & $-.052 \pm 0.102$ \\
\hline & $3 / 16 / 92$ & $3.87 \pm 0.58$ & $2.66 \pm 0.400$ & $-.066 \pm 0.104$ & $-.035 \pm 0.056$ \\
\hline & $6 / 16 / 92$ & $1.98 \pm 0.444$ & $1.38 \pm 0.310$ & $.163 \pm 0.205$ & $.089 \pm 0.112$ \\
\hline & $9 / 21 / 92$ & $3.64 \pm 0.62$ & $2.27 \pm 0.402$ & $-.029 \pm 0.153$ & $-.015 \pm 0.082$ \\
\hline & $12 / 9 / 92$ & $3.04 \pm 1.00$ & $2.15 \pm 0.71$ & $.215 \pm 0.192$ & $.117 \pm 0.106$ \\
\hline QAS-27 & $12 / 9 / 92$ & $4.92 \pm 1.24$ & $3.48 \pm 0.88$ & $.104 \pm 0.210$ & $.055 \pm 0.112$ \\
\hline \multirow[t]{4}{*}{ USGS 102} & $4 / 6 / 93$ & $3.89 \pm 0.70$ & $2.71 \pm 0.490$ & $-.042 \pm 0.072$ & $-.023 \pm 0.039$ \\
\hline & $6 / 9 / 93$ & $3.55 \pm 1.06$ & $2.53 \pm 0.76$ & NR & NR \\
\hline & $9 / 13 / 93$ & $5.42 \pm 1.40$ & $3.89 \pm 1.00$ & NR & NR \\
\hline & $11 / 4 / 93$ & $3.13 \pm 0.98$ & $2.26 \pm 0.71$ & NR & NR \\
\hline \multirow{4}{*}{$\begin{array}{l}\text { Water Supply } \\
\text { INEL-1 }\end{array}$} & $3 / 13 / 91$ & $2.39 \pm 0.404$ & $2.40 \pm 0.407$ & $.227 \pm 0.174$ & $.123 \pm 0.098$ \\
\hline & $6 / 5 / 91$ & $2.64 \pm 0.51$ & $1.82 \pm 0.353$ & $-.120 \pm 0.116$ & $-.063 \pm 0.062$ \\
\hline & $9 / 5 / 91$ & $2.19 \pm 0.468$ & $1.54 \pm 0.330$ & $.226 \pm 0.245$ & $.134 \pm 0.148$ \\
\hline & $12 / 3 / 91$ & $2.72 \pm 0.52$ & $1.69 \pm 0.338$ & $.388 \pm 0.247$ & $.217 \pm 0.144$ \\
\hline QAS-19 & $12 / 3 / 91$ & $3.33 \pm 0.55$ & $2.29 \pm 0.385$ & $-.118 \pm 0.230$ & $-.065 \pm 0.126$ \\
\hline
\end{tabular}


Table 9.-Concentrations of gross alpha-particle radioactivity in water from round-three samples, Naval Reactors Facility and vicinity-Continued

\begin{tabular}{|c|c|c|c|c|c|}
\hline \multirow[b]{2}{*}{$\begin{array}{l}\text { Sample } \\
\text { identifier }\end{array}$} & \multirow{2}{*}{$\begin{array}{c}\text { Date } \\
\text { sampled } \\
(\mathrm{m} / \mathrm{d} / \mathrm{y})\end{array}$} & \multicolumn{2}{|c|}{ Dissolved } & \multicolumn{2}{|c|}{ Suspended } \\
\hline & & $\begin{array}{l}\text { as uranium } \\
\qquad(\mu \mathrm{g} / \mathrm{L})\end{array}$ & $\begin{array}{l}\text { as thorium-230 } \\
\qquad(\mathrm{pCi} / \mathrm{L})\end{array}$ & $\begin{array}{l}\text { as uranium } \\
(\mu \mathrm{g} / \mathrm{L})\end{array}$ & $\begin{array}{l}\text { as thorium-230 } \\
\qquad(\mathrm{pCi} / \mathrm{L})\end{array}$ \\
\hline \multirow{8}{*}{$\begin{array}{l}\text { Water Supply } \\
\text { INEL-1 }\end{array}$} & $3 / 16 / 92$ & $2.87 \pm 0.54$ & $1.80 \pm 0.356$ & $.419 \pm 0.222$ & $.223 \pm 0.124$ \\
\hline & $6 / 16 / 92$ & $2.52 \pm 0.473$ & $1.74 \pm 0.330$ & $-.108 \pm 0.210$ & $-.075 \pm 0.147$ \\
\hline & $9 / 21 / 92$ & $2.75 \pm 0.51$ & $1.92 \pm 0.356$ & $.131 \pm 0.251$ & $.071 \pm 0.138$ \\
\hline & $12 / 8 / 92$ & $3.32 \pm 1.24$ & $2.56 \pm 0.96$ & $-.140 \pm 0.170$ & $-.076 \pm 0.094$ \\
\hline & $4 / 6 / 93$ & $2.15 \pm 0.56$ & $1.48 \pm 0.388$ & $.433 \pm 0.274$ & $.238 \pm 0.156$ \\
\hline & $6 / 8 / 93$ & $1.64 \pm 0.82$ & $1.26 \pm 0.63$ & NR & NR \\
\hline & $9 / 13 / 93$ & $2.54 \pm 1.04$ & $1.96 \pm 0.80$ & NR & NR \\
\hline & $11 / 2 / 93$ & $3.40 \pm 1.21$ & $2.60 \pm 0.925$ & NR & NR \\
\hline
\end{tabular}


Table 10.-Concentrations of gross beta-particle radioactivity in water from round-three samples, Naval Reactors Facility and vicinity-

[Analyses were performed by the U.S. Geological Survey's National Water Quality Laboratory using a residue procedure. Raw field samples were processed in laboratory prior to analysis. Analytical results and uncertainties-for example, 2.45 $\pm \mathbf{0 . 4 1 4}$ - in picocuries per liter.

Analytical uncertainties are reported as 1 s. Concentrations that meet or exceed the reporting level of 3 times the $1 s$ value are shown in boldface type. Abbreviations: $(\mathrm{m} / \mathrm{d} / \mathrm{y})$, month/day/year; Sr-90/Y-90, strontium-90 in equilibrium with yttrium-90. Symbol: NR indicates analysis not requested. Sample identifier: see figure 2 for location of wells; QAS indicates quality-assurance sample, 23 and 30 are blank samples, others are replicates]

\begin{tabular}{|c|c|c|c|c|c|}
\hline \multirow{2}{*}{$\begin{array}{l}\text { Sample } \\
\text { identifier }\end{array}$} & \multirow{2}{*}{$\begin{array}{c}\text { Date } \\
\text { sampled } \\
(\mathrm{m} / \mathrm{d} / \mathrm{y})\end{array}$} & \multicolumn{2}{|c|}{ Dissolved } & \multicolumn{2}{|c|}{ Suspended } \\
\hline & & as $\mathrm{Sr}-90 / \mathrm{Y}-90$ & as cesium-137 & as $\mathrm{Sr}-90 / \mathrm{Y}-90$ & as cesium-137 \\
\hline QAS-23 & $6 / 12 / 92$ & $0.188 \pm 0.164$ & $0.196 \pm 0.172$ & $0.181 \pm 0.238$ & $0.186 \pm 0.246$ \\
\hline QAS-30 & $6 / 15 / 93$ & $-.070 \pm 0.150$ & $-.072 \pm 0.153$ & NR & NR \\
\hline \multirow[t]{3}{*}{ NRF-1 } & $3 / 5 / 91$ & $2.45 \pm 0.414$ & $3.28 \pm 0.56$ & $.480 \pm 0.278$ & $.567 \pm 0.328$ \\
\hline & $6 / 17 / 91$ & $2.45 \pm 0.403$ & $3.23 \pm 0.53$ & $.219 \pm 0.216$ & $.229 \pm 0.226$ \\
\hline & 9/9/91 & $2.45 \pm 0.59$ & $3.33 \pm 0.69$ & $.415 \pm 0.272$ & $.439 \pm 0.289$ \\
\hline QAS-18 & 9/9/91 & $2.76 \pm 0.52$ & $3.59 \pm 0.68$ & $.174 \pm 0.258$ & $.184 \pm 0.273$ \\
\hline \multirow[t]{9}{*}{ NRF-1 } & $12 / 4 / 91$ & $3.02 \pm 0.50$ & $4.03 \pm 0.67$ & $.312 \pm 0.251$ & $.330 \pm 0.266$ \\
\hline & $3 / 11 / 92$ & $2.54 \pm 0.424$ & $3.38 \pm 0.56$ & $.588 \pm 0.258$ & $.623 \pm 0.273$ \\
\hline & $6 / 11 / 92$ & $1.67 \pm 0.326$ & $2.21 \pm 0.432$ & $.279 \pm 0.244$ & $.292 \pm 0.254$ \\
\hline & $9 / 16 / 92$ & $2.19 \pm 0.378$ & $2.89 \pm 0.50$ & $.139 \pm 0.252$ & $.143 \pm 0.258$ \\
\hline & $12 / 3 / 92$ & $2.33 \pm 0.389$ & $3.13 \pm 0.52$ & $.720 \pm 0.274$ & $.764 \pm 0.291$ \\
\hline & $4 / 7 / 93$ & $2.39 \pm 0.430$ & $3.20 \pm 0.58$ & $.453 \pm 0.232$ & $.480 \pm 0.246$ \\
\hline & $6 / 15 / 93$ & $2.54 \pm 0.407$ & $3.37 \pm 0.54$ & NR & NR \\
\hline & $9 / 15 / 93$ & $2.51 \pm 0.411$ & $3.33 \pm 0.54$ & NR & NR \\
\hline & $11 / 4 / 93$ & $3.14 \pm 0.58$ & $3.95 \pm 0.72$ & NR & NR \\
\hline NRF-2 & $3 / 5 / 91$ & $2.92 \pm 0.488$ & $3.86 \pm 0.64$ & $.582 \pm 0.236$ & $.731 \pm 0.296$ \\
\hline QAS-13 & $3 / 5 / 91$ & $2.88 \pm 0.481$ & $3.83 \pm 0.64$ & $.447 \pm 0.249$ & $.467 \pm 0.260$ \\
\hline \multirow[t]{5}{*}{ NRF-2 } & $6 / 17 / 91$ & $2.24 \pm 0.407$ & $3.01 \pm 0.54$ & $.138 \pm 0.215$ & $.147 \pm 0.228$ \\
\hline & 9/9/91 & $2.70 \pm 0.490$ & $3.76 \pm 0.73$ & $.262 \pm 0.240$ & $.270 \pm 0.248$ \\
\hline & $12 / 4 / 91$ & $3.79 \pm 0.58$ & $5.05 \pm 0.77$ & $.116 \pm 0.230$ & $.120 \pm 0.238$ \\
\hline & $3 / 11 / 92$ & $2.99 \pm 0.466$ & $4.00 \pm 0.62$ & $.190 \pm 0.228$ & $.197 \pm 0.236$ \\
\hline & $6 / 11 / 92$ & $2.71 \pm 0.463$ & $3.57 \pm 0.61$ & $.086 \pm 0.232$ & $.088 \pm 0.238$ \\
\hline
\end{tabular}


Table 10.-Concentrations of gross beta-particle radioactivity in water from round-three samples, Naval Reactors Facility and vicinity-Continued

\begin{tabular}{|c|c|c|c|c|c|}
\hline \multirow{2}{*}{$\begin{array}{l}\text { Sample } \\
\text { identifier }\end{array}$} & \multirow{2}{*}{$\begin{array}{c}\text { Date } \\
\text { sampled } \\
(\mathrm{m} / \mathrm{d} / \mathrm{y})\end{array}$} & \multicolumn{2}{|c|}{ Dissolved } & \multicolumn{2}{|c|}{ Suspended } \\
\hline & & as $\mathrm{Sr}-90 / \mathrm{Y}-90$ & as cesium-137 & as $\mathrm{Sr}-90 / \mathrm{Y}-90$ & as cesium-137 \\
\hline \multirow[t]{5}{*}{ NRF-2 } & $9 / 16 / 92$ & $2.60 \pm 0.410$ & $3.48 \pm 0.55$ & $.119 \pm 0.227$ & $.126 \pm 0.240$ \\
\hline & $12 / 3 / 92$ & $3.09 \pm 0.436$ & $4.09 \pm 0.58$ & $.291 \pm 0.265$ & $.300 \pm 0.272$ \\
\hline & $4 / 7 / 93$ & $2.91 \pm 0.470$ & $3.89 \pm 0.63$ & $.400 \pm 0.210$ & $.413 \pm 0.218$ \\
\hline & $6 / 15 / 93$ & $2.80 \pm 0.439$ & $3.76 \pm 0.59$ & NR & NR \\
\hline & $9 / 15 / 93$ & $3.64 \pm 0.488$ & $4.89 \pm 0.65$ & NR & NR \\
\hline QAS-31 & $9 / 15 / 93$ & $3.61 \pm 0.50$ & $4.83 \pm 0.67$ & NR & NR \\
\hline NRF-2 & $11 / 4 / 93$ & $2.83 \pm 0.74$ & $3.70 \pm 0.94$ & NR & NR \\
\hline NRF-3 & $6 / 17 / 91$ & $2.02 \pm 0.378$ & $2.68 \pm 0.50$ & $.078 \pm 0.232$ & $.080 \pm 0.240$ \\
\hline QAS-16 & $6 / 17 / 91$ & $2.20 \pm 0.402$ & $2.94 \pm 0.54$ & $.427 \pm 0.236$ & $.453 \pm 0.250$ \\
\hline \multirow[t]{6}{*}{ NRF-3 } & $9 / 26 / 91$ & $3.09 \pm 0.482$ & $4.12 \pm 0.64$ & $.212 \pm 0.250$ & $.218 \pm 0.258$ \\
\hline & $12 / 4 / 91$ & $3.51 \pm 0.54$ & $4.70 \pm 0.72$ & $.113 \pm 0.242$ & $.117 \pm 0.250$ \\
\hline & $3 / 11 / 92$ & $2.76 \pm 0.412$ & $3.66 \pm 0.54$ & $.453 \pm 0.225$ & $.474 \pm 0.235$ \\
\hline & $6 / 11 / 92$ & $2.31 \pm 0.424$ & $3.10 \pm 0.56$ & $.212 \pm 0.236$ & $.225 \pm 0.250$ \\
\hline & $9 / 16 / 92$ & $2.12 \pm 0.354$ & $2.83 \pm 0.474$ & $4.52 \pm 0.52$ & $4.91 \pm 0.56$ \\
\hline & $12 / 3 / 92$ & $2.38 \pm 0.387$ & $3.14 \pm 0.51$ & $.200 \pm 0.258$ & $.209 \pm 0.270$ \\
\hline QAS-26 & $12 / 3 / 92$ & $2.03 \pm 0.370$ & $2.74 \pm 0.497$ & $.468 \pm 0.284$ & $.483 \pm 0.294$ \\
\hline \multirow[t]{4}{*}{ NRF-3 } & $4 / 8 / 93$ & $2.48 \pm 0.431$ & $3.32 \pm 0.58$ & $.100 \pm 0.229$ & $.103 \pm 0.236$ \\
\hline & $6 / 15 / 93$ & $2.93 \pm 0.442$ & $3.93 \pm 0.60$ & NR & NR \\
\hline & $9 / 15 / 93$ & $3.28 \pm 0.458$ & $4.41 \pm 0.62$ & NR & NR \\
\hline & $11 / 4 / 93$ & $3.04 \pm 0.453$ & $4.04 \pm 0.60$ & NR & NR \\
\hline \multirow[t]{8}{*}{ NRF-4 } & $3 / 5 / 91$ & $3.47 \pm 0.52$ & $4.54 \pm 0.68$ & $.111 \pm 0.222$ & $.141 \pm 0.281$ \\
\hline & $6 / 17 / 91$ & $2.40 \pm 0.411$ & $3.21 \pm 0.55$ & $.295 \pm 0.254$ & $.303 \pm 0.261$ \\
\hline & 9/9/91 & $3.22 \pm 0.53$ & $4.44 \pm 0.82$ & $.098 \pm 0.245$ & $.103 \pm 0.256$ \\
\hline & $12 / 4 / 91$ & $3.17 \pm 0.54$ & $4.23 \pm 0.72$ & $.355 \pm 0.240$ & $.371 \pm 0.252$ \\
\hline & $3 / 11 / 92$ & $3.08 \pm 0.69$ & $4.23 \pm 0.76$ & $.267 \pm 0.236$ & $.283 \pm 0.250$ \\
\hline & $6 / 11 / 92$ & $2.42 \pm 0.402$ & $3.26 \pm 0.54$ & $-.484 \pm 0.224$ & $-.500 \pm 0.231$ \\
\hline & $9 / 16 / 92$ & $3.74 \pm 0.479$ & $5.03 \pm 0.64$ & $.061 \pm 0.230$ & $.063 \pm 0.238$ \\
\hline & $12 / 3 / 92$ & $2.56 \pm 0.403$ & $3.44 \pm 0.54$ & $.434 \pm 0.243$ & $.460 \pm 0.258$ \\
\hline
\end{tabular}


Table 10.-Concentrations of gross beta-particle radioactivity in water from round-three samples, Naval Reactors Facility and vicinity-Continued

\begin{tabular}{|c|c|c|c|c|c|}
\hline \multirow{2}{*}{$\begin{array}{c}\text { Sample } \\
\text { identifier }\end{array}$} & \multirow{2}{*}{$\begin{array}{c}\text { Date } \\
\text { sampled } \\
(\mathrm{m} / \mathrm{d} / \mathrm{y})\end{array}$} & \multicolumn{2}{|c|}{ Dissolved } & \multicolumn{2}{|c|}{ Suspended } \\
\hline & & as $\mathrm{Sr}-90 / \mathrm{Y}-90$ & as cesium-137 & as $\mathrm{Sr}-90 / \mathrm{Y}-90$ & as cesium-137 \\
\hline NRF-4 & $4 / 7 / 93$ & $2.70 \pm 0.425$ & $3.56 \pm 0.56$ & $.250 \pm 0.230$ & $.262 \pm 0.240$ \\
\hline QAS-28 & $4 / 7 / 93$ & $3.76 \pm 0.51$ & $5.00 \pm 0.68$ & $.308 \pm 0.222$ & $.327 \pm 0.234$ \\
\hline \multirow[t]{3}{*}{ NRF-4 } & $6 / 15 / 93$ & $2.78 \pm 0.450$ & $3.73 \pm 0.60$ & NR & NR \\
\hline & $9 / 15 / 93$ & $3.10 \pm 0.444$ & $4.11 \pm 0.59$ & NR & NR \\
\hline & $11 / 4 / 93$ & $3.50 \pm 0.50$ & $4.69 \pm 0.67$ & NR & NR \\
\hline \multirow[t]{5}{*}{ NRF-6 } & $9 / 18 / 92$ & $5.00 \pm 0.80$ & $6.61 \pm 1.06$ & $.388 \pm 0.224$ & $.411 \pm 0.237$ \\
\hline & $12 / 9 / 92$ & $5.43 \pm 0.84$ & $7.28 \pm 1.12$ & $-.042 \pm 0.248$ & $-.043 \pm 0.256$ \\
\hline & $4 / 9 / 93$ & $5.53 \pm 0.92$ & $7.34 \pm 1.22$ & $.177 \pm 0.220$ & $.182 \pm 0.226$ \\
\hline & $9 / 14 / 93$ & $6.83 \pm 0.96$ & $9.01 \pm 1.28$ & NR & NR \\
\hline & $11 / 4 / 93$ & $6.41 \pm 1.19$ & $8.69 \pm 1.73$ & NR & NR \\
\hline \multirow[t]{6}{*}{ NRF-7 } & $9 / 18 / 92$ & $2.29 \pm 0.376$ & $3.07 \pm 0.50$ & $.290 \pm 0.202$ & $.304 \pm 0.212$ \\
\hline & $12 / 9 / 92$ & $2.62 \pm 0.356$ & $3.42 \pm 0.55$ & $.595 \pm 0.254$ & $.631 \pm 0.270$ \\
\hline & $4 / 9 / 93$ & $3.16 \pm 0.52$ & $4.13 \pm 0.61$ & $.666 \pm 0.268$ & $.688 \pm 0.277$ \\
\hline & $6 / 10 / 93$ & $2.71 \pm 0.458$ & $3.49 \pm 0.55$ & NR & NR \\
\hline & $9 / 14 / 93$ & $3.29 \pm 0.398$ & $4.32 \pm 0.64$ & NR & NR \\
\hline & $11 / 3 / 93$ & $3.29 \pm 0.396$ & $4.33 \pm 0.64$ & NR & NR \\
\hline \multirow[t]{2}{*}{ USGS 12} & $6 / 10 / 91$ & $1.79 \pm 0.378$ & $2.36 \pm 0.498$ & $.744 \pm 0.262$ & $.765 \pm 0.270$ \\
\hline & $9 / 6 / 91$ & $2.31 \pm 0.460$ & $2.95 \pm 0.59$ & $.672 \pm 0.263$ & $.693 \pm 0.271$ \\
\hline QAS-17 & $9 / 6 / 91$ & $1.67 \pm 0.400$ & $2.27 \pm 0.57$ & $.418 \pm 0.245$ & $.430 \pm 0.252$ \\
\hline \multirow[t]{9}{*}{ USGS 12} & $12 / 5 / 91$ & $2.70 \pm 0.467$ & $3.54 \pm 0.62$ & $-.215 \pm 0.222$ & $-.221 \pm 0.228$ \\
\hline & $3 / 12 / 92$ & $2.91 \pm 0.456$ & $3.81 \pm 0.60$ & $-.016 \pm 0.240$ & $-.016 \pm 0.246$ \\
\hline & $6 / 19 / 92$ & $2.28 \pm 0.384$ & $3.06 \pm 0.52$ & $-.013 \pm 0.238$ & $-.013 \pm 0.244$ \\
\hline & $9 / 18 / 92$ & $3.06 \pm 0.380$ & $4.07 \pm 0.59$ & $.723 \pm 0.245$ & $.747 \pm 0.253$ \\
\hline & $12 / 1 / 92$ & $1.55 \pm 0.292$ & $2.10 \pm 0.424$ & $2.92 \pm 0.413$ & $3.15 \pm 0.446$ \\
\hline & $4 / 13 / 93$ & $2.09 \pm 0.403$ & $2.80 \pm 0.54$ & $.235 \pm 0.228$ & $.245 \pm 0.238$ \\
\hline & $6 / 14 / 93$ & $2.81 \pm 0.462$ & $3.74 \pm 0.62$ & NR & NR \\
\hline & $9 / 16 / 93$ & $2.85 \pm 0.433$ & $3.73 \pm 0.56$ & NR & NR \\
\hline & $11 / 5 / 93$ & $3.05 \pm 0.80$ & $3.79 \pm 1.02$ & NR & NR \\
\hline
\end{tabular}


Table 10.-Concentrations of gross beta-particle radioactivity in water from round-three samples, Naval Reactors Facility and vicinity-Continued

\begin{tabular}{|c|c|c|c|c|c|}
\hline \multirow{2}{*}{$\begin{array}{l}\text { Sample } \\
\text { identifier }\end{array}$} & \multirow{2}{*}{$\begin{array}{c}\text { Date } \\
\text { sampled } \\
(\mathrm{m} / \mathrm{d} / \mathrm{y})\end{array}$} & \multicolumn{2}{|c|}{ Dissolved } & \multicolumn{2}{|c|}{ Suspended } \\
\hline & & as $\mathrm{Sr}-90 / \mathrm{Y}-90$ & as cesium-137 & as $\mathrm{Sr}-90 / \mathrm{Y}-90$ & as cesium- 137 \\
\hline QAS-33 & $11 / 5 / 93$ & $2.98 \pm 0.431$ & $3.99 \pm 0.58$ & NR & NR \\
\hline \multirow[t]{4}{*}{ USGS 15} & $6 / 10 / 91$ & $1.83 \pm 0.318$ & $2.53 \pm 0.474$ & $.298 \pm 0.232$ & $.311 \pm 0.243$ \\
\hline & $9 / 6 / 91$ & $1.81 \pm 0.386$ & $2.48 \pm 0.56$ & $.003 \pm 0.222$ & $.003 \pm 0.229$ \\
\hline & $12 / 4 / 91$ & $1.69 \pm 0.304$ & $2.29 \pm 0.445$ & $.048 \pm 0.247$ & $.051 \pm 0.262$ \\
\hline & $3 / 12 / 92$ & $1.48 \pm 0.296$ & $1.98 \pm 0.422$ & $.251 \pm 0.234$ & $.266 \pm 0.248$ \\
\hline QAS-22 & $3 / 12 / 92$ & $1.40 \pm 0.286$ & $1.89 \pm 0.410$ & $.303 \pm 0.237$ & $.313 \pm 0.244$ \\
\hline \multirow[t]{7}{*}{ USGS 15} & $6 / 19 / 92$ & $1.90 \pm 0.331$ & $2.48 \pm 0.430$ & $32.2 \pm 4.27$ & $35.4 \pm 4.70$ \\
\hline & $9 / 17 / 92$ & $2.14 \pm 0.345$ & $2.83 \pm 0.50$ & $.520 \pm 0.258$ & $.544 \pm 0.270$ \\
\hline & $12 / 1 / 92$ & $2.69 \pm 0.414$ & $3.62 \pm 0.56$ & $-.187 \pm 0.239$ & $-.193 \pm 0.247$ \\
\hline & $4 / 13 / 93$ & $1.16 \pm 0.274$ & $1.57 \pm 0.388$ & $.262 \pm 0.240$ & $.270 \pm 0.248$ \\
\hline & $6 / 14 / 93$ & $1.79 \pm 0.312$ & $2.44 \pm 0.461$ & NR & NR \\
\hline & $9 / 16 / 93$ & $1.55 \pm 0.296$ & $2.11 \pm 0.432$ & NR & NR \\
\hline & $11 / 5 / 93$ & $2.17 \pm 0.372$ & $2.90 \pm 0.56$ & NR & NR \\
\hline USGS 17 & $3 / 13 / 91$ & $2.12 \pm 0.336$ & $2.86 \pm 0.51$ & $.520 \pm 0.242$ & $.646 \pm 0.300$ \\
\hline QAS-14 & $3 / 13 / 91$ & $2.26 \pm 0.337$ & $3.02 \pm 0.50$ & $.531 \pm 0.250$ & $.672 \pm 0.316$ \\
\hline \multirow[t]{9}{*}{ USGS 17} & $6 / 6 / 91$ & $2.39 \pm 0.458$ & $3.20 \pm 0.53$ & $.236 \pm 0.234$ & $.243 \pm 0.242$ \\
\hline & $9 / 5 / 91$ & $2.54 \pm 0.360$ & $3.42 \pm 0.54$ & $.377 \pm 0.238$ & $.394 \pm 0.249$ \\
\hline & $12 / 3 / 91$ & $2.29 \pm 0.341$ & $3.05 \pm 0.51$ & $.343 \pm 0.246$ & $.354 \pm 0.254$ \\
\hline & $3 / 16 / 92$ & $1.99 \pm 0.320$ & $2.67 \pm 0.471$ & $.592 \pm 0.275$ & $.627 \pm 0.292$ \\
\hline & $6 / 11 / 92$ & $2.16 \pm 0.417$ & $2.85 \pm 0.496$ & $.631 \pm 0.265$ & $.669 \pm 0.281$ \\
\hline & $9 / 16 / 92$ & $2.48 \pm 0.393$ & $3.30 \pm 0.52$ & $.699 \pm 0.249$ & $.740 \pm 0.264$ \\
\hline & $12 / 3 / 92$ & $2.18 \pm 0.421$ & $2.88 \pm 0.50$ & $.412 \pm 0.248$ & $.431 \pm 0.260$ \\
\hline & $4 / 7 / 93$ & $2.73 \pm 0.370$ & $3.66 \pm 0.58$ & $.439 \pm 0.252$ & $.453 \pm 0.261$ \\
\hline & $6 / 11 / 93$ & $2.02 \pm 0.336$ & $2.70 \pm 0.50$ & NR & NR \\
\hline QAS-29 & $6 / 11 / 93$ & $2.32 \pm 0.444$ & $3.08 \pm 0.52$ & NR & NR \\
\hline \multirow[t]{2}{*}{ USGS 17} & $9 / 15 / 93$ & $2.74 \pm 0.480$ & $3.61 \pm 0.56$ & NR & NR \\
\hline & $11 / 8 / 93$ & $2.51 \pm 0.466$ & $3.33 \pm 0.54$ & NR & NR \\
\hline USGS 97 & $3 / 13 / 91$ & $2.59 \pm 0.420$ & $3.48 \pm 0.56$ & $.239 \pm 0.264$ & $.282 \pm 0.312$ \\
\hline
\end{tabular}


Table 10. Concentrations of gross beta-particle radioactivity in water from round-three samples, Naval Reactors Facility and vicinity-Continued

\begin{tabular}{|c|c|c|c|c|c|}
\hline \multirow{2}{*}{$\begin{array}{l}\text { Sample } \\
\text { identifier }\end{array}$} & \multirow{2}{*}{$\begin{array}{c}\text { Date } \\
\text { sampled } \\
(\mathrm{m} / \mathrm{d} / \mathrm{y})\end{array}$} & \multicolumn{2}{|c|}{ Dissolved } & \multicolumn{2}{|c|}{ Suspended } \\
\hline & & as $\mathrm{Sr}-90 / \mathrm{Y}-90$ & as cesium-137 & as $\mathrm{Sr}-90 / \mathrm{Y}-90$ & as cesium- 137 \\
\hline USGS 97 & $6 / 7 / 91$ & $3.20 \pm 0.488$ & $4.25 \pm 0.65$ & $.360 \pm 0.223$ & $.381 \pm 0.236$ \\
\hline QAS-15 & $6 / 7 / 91$ & $2.36 \pm 0.466$ & $3.09 \pm 0.61$ & $.409 \pm 0.240$ & $.427 \pm 0.252$ \\
\hline \multirow[t]{10}{*}{ USGS 97} & $9 / 5 / 91$ & $2.75 \pm 0.496$ & $3.75 \pm 0.73$ & $.419 \pm 0.255$ & $.438 \pm 0.266$ \\
\hline & $12 / 3 / 91$ & $2.50 \pm 0.475$ & $3.29 \pm 0.62$ & $.269 \pm 0.248$ & $.277 \pm 0.256$ \\
\hline & $3 / 16 / 92$ & $2.18 \pm 0.371$ & $2.88 \pm 0.490$ & $.380 \pm 0.246$ & $.398 \pm 0.258$ \\
\hline & $6 / 17 / 92$ & $3.04 \pm 0.446$ & $4.03 \pm 0.60$ & $-.242 \pm 0.240$ & $-.250 \pm 0.248$ \\
\hline & $9 / 21 / 92$ & $3.07 \pm 0.430$ & $4.06 \pm 0.57$ & $.513 \pm 0.230$ & $.529 \pm 0.237$ \\
\hline & $12 / 8 / 92$ & $2.13 \pm 0.368$ & $2.82 \pm 0.486$ & $.314 \pm 0.244$ & $.323 \pm 0.250$ \\
\hline & $4 / 6 / 93$ & $2.20 \pm 0.420$ & $2.88 \pm 0.55$ & $-.029 \pm 0.240$ & $-.031 \pm 0.254$ \\
\hline & $6 / 9 / 93$ & $2.08 \pm 0.406$ & $2.73 \pm 0.54$ & NR & NR \\
\hline & $9 / 13 / 93$ & $2.96 \pm 0.468$ & $3.93 \pm 0.62$ & NR & NR \\
\hline & $11 / 4 / 93$ & $3.14 \pm 0.437$ & $4.15 \pm 0.58$ & NR & NR \\
\hline QAS-32 & $11 / 4 / 93$ & $2.66 \pm 0.440$ & $3.53 \pm 0.58$ & NR & NR \\
\hline \multirow[t]{7}{*}{ USGS 98} & $3 / 13 / 91$ & $2.98 \pm 0.409$ & $3.90 \pm 0.54$ & $.495 \pm 0.278$ & $.524 \pm 0.294$ \\
\hline & $6 / 7 / 91$ & $2.22 \pm 0.358$ & $2.95 \pm 0.475$ & $.312 \pm 0.219$ & $.330 \pm 0.232$ \\
\hline & $9 / 5 / 91$ & $1.83 \pm 0.444$ & $2.49 \pm 0.52$ & $.368 \pm 0.253$ & $.378 \pm 0.260$ \\
\hline & $12 / 3 / 91$ & $2.32 \pm 0.357$ & $3.21 \pm 0.56$ & $.241 \pm 0.260$ & $.256 \pm 0.276$ \\
\hline & $3 / 16 / 92$ & $1.96 \pm 0.447$ & $2.69 \pm 0.50$ & $.174 \pm 0.261$ & $.179 \pm 0.268$ \\
\hline & $6 / 16 / 92$ & $2.31 \pm 0.340$ & $3.17 \pm 0.52$ & $-.274 \pm 0.238$ & $-.283 \pm 0.246$ \\
\hline & $9 / 21 / 92$ & $2.74 \pm 0.56$ & $3.76 \pm 0.59$ & $.463 \pm 0.247$ & $.490 \pm 0.262$ \\
\hline QAS-25 & $9 / 21 / 92$ & $2.72 \pm 0.397$ & $3.63 \pm 0.53$ & $.607 \pm 0.227$ & $.635 \pm 0.238$ \\
\hline \multirow[t]{3}{*}{ USGS 98} & $12 / 8 / 92$ & $2.76 \pm 0.378$ & $3.81 \pm 0.59$ & $.504 \pm 0.256$ & $.518 \pm 0.264$ \\
\hline & $4 / 6 / 93$ & $2.19 \pm 0.491$ & $3.01 \pm 0.53$ & $.517 \pm 0.236$ & $.548 \pm 0.251$ \\
\hline & $6 / 8 / 93$ & $2.13 \pm 0.356$ & $2.78 \pm 0.464$ & NR & NR \\
\hline \multirow[t]{4}{*}{ USGS 99} & $3 / 13 / 91$ & $1.71 \pm 0.360$ & $2.26 \pm 0.476$ & $.407 \pm 0.224$ & $.511 \pm 0.282$ \\
\hline & $6 / 7 / 91$ & $2.34 \pm 0.416$ & $3.14 \pm 0.56$ & $.488 \pm 0.246$ & $.510 \pm 0.257$ \\
\hline & $9 / 5 / 91$ & $2.31 \pm 0.432$ & $3.20 \pm 0.64$ & $.087 \pm 0.224$ & $.092 \pm 0.238$ \\
\hline & $12 / 3 / 91$ & $2.08 \pm 0.412$ & $2.76 \pm 0.54$ & $.514 \pm 0.252$ & $.537 \pm 0.263$ \\
\hline
\end{tabular}


Table 10.-Concentrations of gross beta-particle radioactivity in water from round-three samples, Naval Reactors Facility and vicinity-Continued

\begin{tabular}{|c|c|c|c|c|c|}
\hline \multirow{2}{*}{$\begin{array}{l}\text { Sample } \\
\text { identifier }\end{array}$} & \multirow{2}{*}{$\begin{array}{c}\text { Date } \\
\text { sampled } \\
(\mathrm{m} / \mathrm{d} / \mathrm{y})\end{array}$} & \multicolumn{2}{|c|}{ Dissolved } & \multicolumn{2}{|c|}{ Suspended } \\
\hline & & as $\mathrm{Sr}-90 / \mathrm{Y}-90$ & as cesium-137 & as $\mathrm{Sr}-90 / \mathrm{Y}-90$ & as cesium-137 \\
\hline \multirow[t]{2}{*}{ USGS 99} & $3 / 16 / 92$ & $1.89 \pm 0.334$ & $2.53 \pm 0.446$ & $-.063 \pm 0.246$ & $-.065 \pm 0.254$ \\
\hline & $6 / 16 / 92$ & $1.70 \pm 0.339$ & $2.25 \pm 0.446$ & $.438 \pm 0.254$ & $.457 \pm 0.266$ \\
\hline QAS-24 & $6 / 16 / 92$ & $1.82 \pm 0.339$ & $2.43 \pm 0.454$ & $.453 \pm 0.252$ & $.480 \pm 0.268$ \\
\hline \multirow[t]{6}{*}{ USGS 99} & $9 / 21 / 92$ & $1.94 \pm 0.352$ & $2.55 \pm 0.462$ & $-.260 \pm 0.228$ & $-.267 \pm 0.234$ \\
\hline & $12 / 8 / 92$ & $1.40 \pm 0.312$ & $1.88 \pm 0.418$ & $.274 \pm 0.272$ & $.283 \pm 0.281$ \\
\hline & $4 / 6 / 93$ & $1.83 \pm 0.364$ & $2.45 \pm 0.485$ & $.266 \pm 0.230$ & $.278 \pm 0.241$ \\
\hline & $6 / 9 / 93$ & $2.40 \pm 0.382$ & $3.22 \pm 0.52$ & NR & NR \\
\hline & $9 / 13 / 93$ & $2.64 \pm 0.400$ & $3.47 \pm 0.52$ & NR & NR \\
\hline & $11 / 2 / 93$ & $2.33 \pm 0.398$ & $3.04 \pm 0.52$ & NR & NR \\
\hline \multirow[t]{7}{*}{ USGS 102} & $6 / 7 / 91$ & $2.95 \pm 0.442$ & $3.95 \pm 0.59$ & $.586 \pm 0.250$ & $.602 \pm 0.258$ \\
\hline & $9 / 5 / 91$ & $2.67 \pm 0.458$ & $3.73 \pm 0.70$ & $.229 \pm 0.238$ & $.237 \pm 0.246$ \\
\hline & $12 / 3 / 91$ & $3.23 \pm 0.52$ & $4.28 \pm 0.68$ & $.076 \pm 0.248$ & $.080 \pm 0.259$ \\
\hline & $3 / 16 / 92$ & $2.53 \pm 0.433$ & $3.34 \pm 0.57$ & $-.048 \pm 0.242$ & $-.050 \pm 0.250$ \\
\hline & $6 / 16 / 92$ & $2.43 \pm 0.410$ & $3.20 \pm 0.54$ & $-.070 \pm 0.220$ & $-.073 \pm 0.230$ \\
\hline & $9 / 21 / 92$ & $3.22 \pm 0.439$ & $4.28 \pm 0.58$ & $.389 \pm 0.225$ & $.400 \pm 0.232$ \\
\hline & $12 / 9 / 92$ & $2.18 \pm 0.372$ & $2.88 \pm 0.490$ & $.022 \pm 0.248$ & $.023 \pm 0.255$ \\
\hline QAS-27 & $12 / 9 / 92$ & $2.44 \pm 0.384$ & $3.25 \pm 0.51$ & $.231 \pm 0.260$ & $.242 \pm 0.272$ \\
\hline \multirow[t]{4}{*}{ USGS 102} & $4 / 6 / 93$ & $2.49 \pm 0.423$ & $3.34 \pm 0.56$ & $.095 \pm 0.237$ & $.099 \pm 0.248$ \\
\hline & $6 / 9 / 93$ & $2.73 \pm 0.423$ & $3.62 \pm 0.56$ & NR & NR \\
\hline & $9 / 13 / 93$ & $2.57 \pm 0.418$ & $3.37 \pm 0.55$ & NR & NR \\
\hline & $11 / 4 / 93$ & $3.06 \pm 0.480$ & $4.06 \pm 0.64$ & NR & NR \\
\hline \multirow{4}{*}{$\begin{array}{l}\text { Water Supply } \\
\text { INEL-1 }\end{array}$} & $3 / 13 / 91$ & $4.33 \pm 0.66$ & $5.80 \pm 0.88$ & $.781 \pm 0.274$ & $.807 \pm 0.283$ \\
\hline & $6 / 5 / 91$ & $3.63 \pm 0.58$ & $4.82 \pm 0.77$ & $.446 \pm 0.249$ & $.460 \pm 0.257$ \\
\hline & $9 / 5 / 91$ & $3.78 \pm 0.60$ & $4.95 \pm 0.78$ & $.244 \pm 0.244$ & $.255 \pm 0.254$ \\
\hline & $12 / 3 / 91$ & $3.78 \pm 0.63$ & $4.99 \pm 0.83$ & $.054 \pm 0.240$ & $.055 \pm 0.247$ \\
\hline QAS-19 & $12 / 3 / 91$ & $4.04 \pm 0.64$ & $5.44 \pm 0.86$ & $.371 \pm 0.260$ & $.384 \pm 0.271$ \\
\hline $\begin{array}{l}\text { Water Supply } \\
\text { INEL-1 }\end{array}$ & $3 / 16 / 92$ & $3.45 \pm 0.53$ & $4.58 \pm 0.70$ & $.269 \pm 0.240$ & $.282 \pm 0.250$ \\
\hline
\end{tabular}


Table 10.-Concentrations of gross beta-particle radioactivity in water from round-three samples, Naval Reactors Facility and vicinity-Continued

\begin{tabular}{|c|c|c|c|c|c|}
\hline \multirow{2}{*}{$\begin{array}{l}\text { Sample } \\
\text { identifier }\end{array}$} & \multirow{2}{*}{$\begin{array}{c}\text { Date } \\
\text { sampled } \\
(\mathrm{m} / \mathrm{d} / \mathrm{y})\end{array}$} & \multicolumn{2}{|c|}{ Dissolved } & \multicolumn{2}{|c|}{ Suspended } \\
\hline & & as $\mathrm{Sr}-90 / \mathrm{Y}-90$ & as cesium-137 & as $\mathrm{Sr}-90 / \mathrm{Y}-90$ & as cesium-137 \\
\hline \multirow{7}{*}{$\begin{array}{l}\text { Water Supply } \\
\text { INEL-1 }\end{array}$} & $6 / 16 / 92$ & $2.54 \pm 0.448$ & $3.40 \pm 0.60$ & $.292 \pm 0.246$ & $.301 \pm 0.254$ \\
\hline & $9 / 21 / 92$ & $3.61 \pm 0.50$ & $4.79 \pm 0.67$ & $.872 \pm 0.270$ & $.911 \pm 0.283$ \\
\hline & $12 / 8 / 92$ & $3.29 \pm 0.480$ & $4.42 \pm 0.64$ & $.736 \pm 0.275$ & $.780 \pm 0.292$ \\
\hline & $4 / 6 / 93$ & $3.70 \pm 0.55$ & $4.86 \pm 0.72$ & $.791 \pm 0.287$ & $.817 \pm 0.296$ \\
\hline & $6 / 8 / 93$ & $3.43 \pm 0.496$ & $4.55 \pm 0.66$ & NR & NR \\
\hline & $9 / 13 / 93$ & $3.96 \pm 0.54$ & $5.30 \pm 0.73$ & NR & NR \\
\hline & $11 / 2 / 93$ & $2.82 \pm 0.55$ & $3.66 \pm 0.71$ & NR & NR \\
\hline
\end{tabular}


Table 11. Statistical parameters for gross alpha-particle radioactivity, gross beta-particle radioactivity, radium-226, and radium-228, by well-

[See figure 2 for location of wells. Gross alpha-particle radioactivity is expressed as uranium in micrograms per liter and thorium-230 in picocuries per liter. All other units are picocuries per liter. Gross beta-particle radioactivity is expressed as strontium-90 in equilibrium with yttrium-90 and as cesium-137. Values are derived from tables 9, 10, 17, and 23. Qualityassurance replicates are included in the calculation of statistical parameters. See section on calculation of estimated experimental standard error for the estimated uncertainty of the mean concentration. Abbreviation: $\mathrm{Sr}-90 / \mathrm{Y}-90$, strontium-90 in equilibrium with yttrium-90]

\begin{tabular}{|c|c|c|c|c|c|}
\hline \multirow{2}{*}{$\begin{array}{l}\text { Radioactivity or } \\
\text { radionuclide }\end{array}$} & \multicolumn{5}{|c|}{ Statistical parameter } \\
\hline & Minimum & Maximum & Median & Mean & $\begin{array}{c}\text { Sample } \\
\text { size }\end{array}$ \\
\hline & \multicolumn{5}{|c|}{ NRF-1 } \\
\hline Uranium (dissolved) & $1.77 \pm 0.394$ & $6.76 \pm 1.52$ & $3.83 \pm 1.15$ & $3.89 \pm 0.416$ & 13 \\
\hline Thorium-230 (dissolved) & $1.23 \pm 0.276$ & $5.01 \pm 1.12$ & $2.78 \pm 0.423$ & $2.83 \pm 0.311$ & 13 \\
\hline Uranium (suspended) & $-.119 \pm 0.116$ & $.636 \pm 0.346$ & $.0285 \pm 0.145$ & $.125 \pm 0.086$ & 10 \\
\hline Thorium-230 (suspended) & $-.064 \pm 0.062$ & $.370 \pm 0.204$ & $.0155 \pm 0.078$ & $.071 \pm 0.049$ & 10 \\
\hline SR-90/Y-90 (dissolved) & $1.67 \pm 0.326$ & $3.14 \pm 0.58$ & $2.45 \pm 0.403$ & $2.50 \pm 0.101$ & 13 \\
\hline Cesium-137 (dissolved) & $2.21 \pm 0.432$ & $4.03 \pm 0.67$ & $3.33 \pm 0.69$ & $3.30 \pm 0.125$ & 13 \\
\hline SR-90/Y-90 (suspended) & $.139 \pm 0.252$ & $.720 \pm 0.274$ & $.3635 \pm 0.185$ & $.378 \pm 0.059$ & 10 \\
\hline \multirow[t]{2}{*}{ Cesium-137 (suspended) } & $.143 \pm 0.258$ & $.764 \pm 0.291$ & $.3845 \pm 0.196$ & $.405 \pm 0.065$ & 10 \\
\hline & \multicolumn{5}{|c|}{ NRF-2 } \\
\hline Uranium (dissolved) & $2.28 \pm 0.450$ & $5.73 \pm 1.50$ & $3.91 \pm 0.66$ & $4.02 \pm 0.259$ & 14 \\
\hline Thorium-230 (dissolved) & $1.58 \pm 0.314$ & $4.42 \pm 1.15$ & $2.87 \pm 0.322$ & $2.97 \pm 0.184$ & 14 \\
\hline Uranium (suspended) & $-.274 \pm 0.124$ & $.539 \pm 0.298$ & $-.075 \pm 0.080$ & $-.017 \pm 0.070$ & 10 \\
\hline Thorium-230 (suspended) & $-.158 \pm 0.077$ & $.291 \pm 0.164$ & $-.040 \pm 0.043$ & $-.010 \pm 0.038$ & 10 \\
\hline SR-90/Y-90 (dissolved) & $2.24 \pm 0.407$ & $3.79 \pm 0.58$ & $2.895 \pm 0.336$ & $2.98 \pm 0.115$ & 14 \\
\hline Cesium-137 (dissolved) & $3.01 \pm 0.54$ & $5.05 \pm 0.77$ & $3.845 \pm 0.454$ & $3.98 \pm 0.154$ & 14 \\
\hline SR-90/Y-90 (suspended) & $.086 \pm 0.232$ & $.582 \pm 0.236$ & $.226 \pm 0.166$ & $.263 \pm 0.053$ & 10 \\
\hline \multirow[t]{2}{*}{ Cesium-137 (suspended) } & $.088 \pm 0.238$ & $.731 \pm 0.296$ & $.2335 \pm 0.171$ & $.286 \pm 0.066$ & 10 \\
\hline & \multicolumn{5}{|c|}{ NRF-3 } \\
\hline Uranium (dissolved) & $1.53 \pm 0.345$ & $6.17 \pm 1.48$ & $3.66 \pm 0.60$ & $3.94 \pm 0.333$ & 13 \\
\hline Thorium-230 (dissolved) & $1.01 \pm 0.228$ & $3.95 \pm 0.96$ & $2.44 \pm 0.73$ & $2.71 \pm 0.257$ & 13 \\
\hline Uranium (suspended) & $-.348 \pm 0.164$ & $6.58 \pm 1.84$ & $.074 \pm 0.108$ & $.660 \pm 0.66$ & 10 \\
\hline Thorium-230 (suspended) & $-.200 \pm 0.096$ & $5.08 \pm 1.60$ & $.0415 \pm 0.060$ & $.508 \pm 0.51$ & 10 \\
\hline SR-90/Y-90 (dissolved) & $2.02 \pm 0.378$ & $3.51 \pm 0.54$ & $2.48 \pm 0.431$ & $2.63 \pm 0.140$ & 13 \\
\hline Cesium-137 (dissolved) & $2.68 \pm 0.50$ & $4.70 \pm 0.72$ & $3.32+0.58$ & $3.51 \pm 0.188$ & 13 \\
\hline
\end{tabular}


Table 11.-Statistical parameters for gross alpha-particle radioactivity, gross beta-particle radioactivity, radium-226, and radium-228, by well-Continued

\begin{tabular}{|c|c|c|c|c|c|}
\hline \multirow{2}{*}{$\begin{array}{l}\text { Radioactivity or } \\
\text { radionuclide }\end{array}$} & \multicolumn{5}{|c|}{ Statistical parameter } \\
\hline & Minimum & Maximum & Median & Mean & $\begin{array}{c}\text { Sample } \\
\text { size }\end{array}$ \\
\hline & \multicolumn{5}{|c|}{ NRF-3 - cont. } \\
\hline SR-90/Y-90 (suspended) & $.078 \pm 0.232$ & $4.52 \pm 0.52$ & $.212 \pm 0.172$ & $.678 \pm 0.429$ & 10 \\
\hline \multirow[t]{2}{*}{ Cesium-137 (suspended) } & $.080 \pm 0.240$ & $4.91 \pm 0.56$ & $.2215 \pm 0.180$ & $.727 \pm 0.467$ & 10 \\
\hline & \multicolumn{5}{|c|}{ NRF-4 } \\
\hline Uranium (dissolved) & $1.35 \pm 0.68$ & $6.48 \pm 1.70$ & $3.77 \pm 0.58$ & $3.63 \pm 0.366$ & 13 \\
\hline Thorium-230 (dissolved) & $.972 \pm 0.240$ & $4.11 \pm 1.08$ & $2.69 \pm 0.424$ & $2.54 \pm 0.234$ & 13 \\
\hline Uranium (suspended) & $-.345 \pm 0.180$ & $.401 \pm 0.212$ & $.061 \pm 0.133$ & $.056 \pm 0.060$ & 10 \\
\hline Thorium-230 (suspended) & $-.190 \pm 0.103$ & $.218 \pm 0.120$ & $.033 \pm 0.073$ & $.030 \pm 0.028$ & 10 \\
\hline SR-90/Y-90 (dissolved) & $2.40 \pm 0.411$ & $3.76 \pm 0.51$ & $3.10 \pm 0.444$ & $3.07 \pm 0.130$ & 13 \\
\hline Cesium-137 (dissolved) & $3.21 \pm 0.55$ & $5.03 \pm 0.64$ & $4.23 \pm 0.76$ & $4.11 \pm 0.174$ & 13 \\
\hline SR-90/Y-90 (suspended) & $-.484 \pm 0.224$ & $.434 \pm 0.243$ & $.2585 \pm 0.165$ & $.170 \pm 0.082$ & 10 \\
\hline \multirow[t]{2}{*}{ Cesium-137 (suspended) } & $-.500 \pm 0.231$ & $.460 \pm 0.258$ & $.2725 \pm 0.173$ & $.181 \pm 0.085$ & 10 \\
\hline & \multicolumn{5}{|c|}{ NRF-6 } \\
\hline Uranium (dissolved) & $2.88 \pm 1.72$ & $7.03 \pm 2.74$ & $4.14 \pm 0.440$ & $4.28 \pm 0.355$ & 12 \\
\hline Thorium-230 (dissolved) & $1.97 \pm 0.352$ & $5.22 \pm 2.04$ & $2.89 \pm 0.309$ & $3.04 \pm 0.271$ & 12 \\
\hline Uranium (suspended) - & $-.135 \pm 0.164$ & $.216 \pm 0.194$ & $.0895 \pm 0.131$ & $.050 \pm 0.035$ & 10 \\
\hline Thorium-230 (suspended) & $-.076 \pm 0.092$ & $.116 \pm 0.106$ & $.047 \pm 0.070$ & $.027 \pm 0.019$ & 10 \\
\hline SR-90/Y-90 (dissolved) & $1.85 \pm 0.68$ & $6.83 \pm 0.96$ & $5.465 \pm 0.60$ & $5.16 \pm 0.354$ & 12 \\
\hline Cesium-137 (dissolved) & $2.43 \pm 0.89$ & $9.01 \pm 1.28$ & $7.055 \pm 0.75$ & $6.79 \pm 0.480$ & 12 \\
\hline SR-90/Y-90 (suspended) & $-.042 \pm 0.248$ & $.520 \pm 0.238$ & $.2105 \pm 0.167$ & $.216 \pm 0.061$ & 10 \\
\hline Cesium-137 (suspended) & $-.043 \pm 0.256$ & $.537 \pm 0.246$ & $.2235 \pm 0.177$ & $.226 \pm 0.064$ & 10 \\
\hline Radium-226 (dissolved) & $.013 \pm 0.004$ & $.052 \pm 0.007$ & $.029 \pm 0.004$ & $.030 \pm 0.005$ & 7 \\
\hline \multirow[t]{2}{*}{ Radium-228 (dissolved) } & $-.044 \pm 0.121$ & $.259 \pm 0.143$ & $.197 \pm 0.134$ & $.167 \pm 0.039$ & 7 \\
\hline & \multicolumn{5}{|c|}{ NRF-7 } \\
\hline Uranium (dissolved) & $.833 \pm 0.268$ & $3.37 \pm 0.54$ & $1.98 \pm 0.422$ & $2.05 \pm 0.197$ & 13 \\
\hline Thorium-230 (dissolved) & $.553 \pm 0.178$ & $2.32 \pm 0.368$ & $1.37 \pm 0.416$ & $1.43 \pm 0.145$ & 13 \\
\hline Uranium (suspended) & $-.134 \pm 0.162$ & $.494 \pm 0.242$ & $.1415 \pm 0.148$ & $.156 \pm 0.054$ & 10 \\
\hline Thorium-230 (suspended) & $-.082 \pm 0.101$ & $.408 \pm 0.214$ & $.0975 \pm 0.087$ & $.112 \pm 0.041$ & 10 \\
\hline SR-90/Y-90 (dissolved) & $2.27 \pm 0.335$ & $3.29 \pm 0.396$ & $2.71 \pm 0.458$ & $2.77 \pm 0.094$ & 13 \\
\hline Cesium-137 (dissolved) & $3.03 \pm 0.498$ & $4.33 \pm 0.64$ & $3.49 \pm 0.55$ & $3.65 \pm 0.123$ & 13 \\
\hline
\end{tabular}


Table 11. Statistical parameters for gross alpha-particle radioactivity, gross beta-particle radioactivity, radium-226, and radium-228, by well-Continued

\begin{tabular}{|c|c|c|c|c|c|}
\hline \multirow{2}{*}{$\begin{array}{l}\text { Radioactivity or } \\
\text { radionuclide }\end{array}$} & \multicolumn{5}{|c|}{ Statistical parameter } \\
\hline & Minimum & Maximum & Median & Mean & $\begin{array}{l}\text { Sample } \\
\text { size }\end{array}$ \\
\hline & \multicolumn{5}{|c|}{ NRF-7 - cont. } \\
\hline SR-90/Y-90 (suspended) & $.290 \pm 0.202$ & $.928 \pm 0.286$ & $.6145 \pm 0.184$ & $.606 \pm 0.063$ & 10 \\
\hline Cesium-137 (suspended) & $.304 \pm 0.212$ & $.963 \pm 0.297$ & $.643 \pm 0.193$ & $.631 \pm 0.062$ & 10 \\
\hline Radium-226 (dissolved) & $.033 \pm 0.006$ & $.047 \pm 0.006$ & $.042 \pm 0.007$ & $.041 \pm 0.002$ & 7 \\
\hline \multirow[t]{2}{*}{ Radium-228 (dissolved) } & $.110 \pm 0.122$ & $.328 \pm 0.152$ & $.189 \pm 0.120$ & $.216 \pm 0.033$ & 7 \\
\hline & \multicolumn{5}{|c|}{ USGS 12} \\
\hline Uranium (dissolved) & $1.51 \pm 0.390$ & $5.38 \pm 1.39$ & $3.46 \pm 0.585$ & $3.66 \pm 0.321$ & 13 \\
\hline Thorium-230 (dissolved) & $1.05 \pm 0.272$ & $3.85 \pm 1.00$ & $2.41 \pm 0.410$ & $2.57 \pm 0.237$ & 13 \\
\hline Uranium (suspended) & $-.212 \pm 0.214$ & $3.75 \pm 1.06$ & $.086 \pm 0.130$ & $.487 \pm 0.411$ & 9 \\
\hline Thorium-230 (suspended) & $-.112 \pm 0.114$ & $3.72 \pm 1.06$ & $.045 \pm 0.070$ & $.452 \pm 0.413$ & 9 \\
\hline SR-90/Y-90 (dissolved) & $1.55 \pm 0.292$ & $3.06 \pm 0.380$ & $2.70 \pm 0.467$ & $2.47 \pm 0.152$ & 13 \\
\hline Cesium-137 (dissolved) & $2.10 \pm 0.424$ & $4.07 \pm 0.59$ & $3.54 \pm 0.62$ & $3.25 \pm 0.193$ & 13 \\
\hline SR-90/Y-90 (suspended) & $-.215 \pm 0.222$ & $2.92 \pm 0.413$ & $.418 \pm 0.245$ & $.608 \pm 0.312$ & 9 \\
\hline \multirow[t]{2}{*}{ Cesium-137 (suspended) } & $-.221 \pm 0.228$ & $3.15 \pm 0.446$ & $.430 \pm 0.252$ & $.642 \pm 0.336$ & 9 \\
\hline & \multicolumn{5}{|c|}{ USGS 15} \\
\hline Uranium (dissolved) & $1.62 \pm 0.380$ & $3.96 \pm 1.24$ & $2.285 \pm 0.440$ & $2.63 \pm 0.245$ & 12 \\
\hline Thorium-230 (dissolved) & $1.12 \pm 0.263$ & $3.06 \pm 0.95$ & $1.595 \pm 0.316$ & $1.87 \pm 0.199$ & 12 \\
\hline Uranium (suspended) & $-.236 \pm 0.144$ & $29.0 \pm 14.9$ & $.028 \pm 0.251$ & $3.28 \pm 3.21$ & 9 \\
\hline Thorium-230 (suspended) & $-.126 \pm 0.080$ & $22.4 \pm 11.4$ & $.016 \pm 0.142$ & $2.52 \pm 2.48$ & 9 \\
\hline SR-90/Y-90 (dissolved) & $1.16 \pm 0.278$ & $2.69 \pm 0.414$ & $1.80 \pm 0.248$ & $1.80 \pm 0.117$ & 12 \\
\hline Cesium-137 (dissolved) & $1.57 \pm 0.388$ & $3.62 \pm 0.56$ & $2.46 \pm 0.365$ & $2.43 \pm 0.155$ & 12 \\
\hline SR-90/Y-90 (suspended) & $-.187 \pm 0.239$ & $32.2 \pm 4.27$ & $.262 \pm 0.240$ & $3.74 \pm 3.57$ & 9 \\
\hline \multirow[t]{2}{*}{ Cesium-137 (suspended) } & $-.193 \pm 0.247$ & $35.4 \pm 4.70$ & $.270 \pm 0.248$ & $4.11 \pm 3.91$ & 9 \\
\hline & \multicolumn{5}{|c|}{ USGS 17} \\
\hline Uranium (dissolved) & $1.26 \pm 0.292$ & $4.11 \pm 0.62$ & $2.065 \pm 0.306$ & $2.25 \pm 0.196$ & 14 \\
\hline Thorium-230 (dissolved) & $.931 \pm 0.244$ & $2.94 \pm 0.443$ & $1.435 \pm 0.283$ & $1.62 \pm 0.129$ & 14 \\
\hline Uranium (suspended) & $-.292 \pm 0.154$ & $.365 \pm 0.208$ & $.0625 \pm 0.153$ & $.063 \pm 0.059$ & 10 \\
\hline Thorium-230 (suspended) & $-.157 \pm 0.088$ & $.197 \pm 0.117$ & $.0385 \pm 0.094$ & $.036 \pm 0.032$ & 10 \\
\hline SR-90/Y-90 (dissolved) & $1.99 \pm 0.320$ & $2.74 \pm 0.480$ & $2.305 \pm 0.280$ & $2.34 \pm 0.064$ & 14 \\
\hline Cesium-137 (dissolved) & $2.67 \pm 0.471$ & $3.66 \pm 0.58$ & $3.065 \pm 0.364$ & $3.12 \pm 0.084$ & 14 \\
\hline
\end{tabular}


Table 11.-Statistical parameters for gross alpha-particle radioactivity, gross beta-particle radioactivity, radium-226, and radium-228, by well-Continued

\begin{tabular}{|c|c|c|c|c|c|}
\hline \multirow{2}{*}{$\begin{array}{l}\text { Radioactivity or } \\
\text { radionuclide }\end{array}$} & \multicolumn{5}{|c|}{ Statistical parameter } \\
\hline & Minimum & Maximum & Median & Mean & $\begin{array}{l}\text { Sample } \\
\text { size }\end{array}$ \\
\hline & \multicolumn{5}{|c|}{ USGS 17 - cont. } \\
\hline SR-90/Y-90 (suspended) & $.236 \pm 0.234$ & $.699 \pm 0.249$ & $.4795 \pm 0.175$ & $.478 \pm 0.045$ & 10 \\
\hline \multirow[t]{2}{*}{ Cesium-137 (suspended) } & $.243 \pm 0.242$ & $.740 \pm 0.264$ & $.540 \pm 0.196$ & $.523 \pm 0.053$ & 10 \\
\hline & \multicolumn{5}{|c|}{ USGS 97} \\
\hline Uranium (dissolved) & $2.15 \pm 0.469$ & $5.93 \pm 1.30$ & $3.45 \pm 0.398$ & $3.82 \pm 0.347$ & 14 \\
\hline Thorium-230 (dissolved) & $1.34 \pm 0.302$ & $4.19 \pm 1.00$ & $2.575 \pm 0.282$ & $2.75 \pm 0.248$ & 14 \\
\hline Uranium (suspended) & $-.305 \pm 0.180$ & $.280 \pm 0.284$ & $.0285 \pm 0.098$ & $.050 \pm 0.052$ & 10 \\
\hline Thorium-230 (suspended) & $-.162 \pm 0.100$ & $.150 \pm 0.153$ & $.0155 \pm 0.056$ & $.027 \pm 0.028$ & 10 \\
\hline SR-90/Y-90 (dissolved) & $2.08 \pm 0.406$ & $3.20 \pm 0.488$ & $2.625 \pm 0.304$ & $2.63 \pm 0.107$ & 14 \\
\hline Cesium-137 (dissolved) & $2.73 \pm 0.54$ & $4.25 \pm 0.65$ & $3.505 \pm 0.403$ & $3.49 \pm 0.146$ & 14 \\
\hline SR-90/Y-90 (suspended) & $-.242 \pm 0.240$ & $.513 \pm 0.230$ & $.337 \pm 0.165$ & $.263 \pm 0.073$ & 10 \\
\hline \multirow[t]{2}{*}{ Cesium-137 (suspended) } & $-.250 \pm 0.248$ & $.529 \pm 0.237$ & $.352 \pm 0.172$ & $.277 \pm 0.076$ & 10 \\
\hline & \multicolumn{5}{|c|}{ USGS 98} \\
\hline Uranium (dissolved) & $1.49 \pm 0.356$ & $3.44 \pm 0.96$ & $2.45 \pm 0.488$ & $2.52 \pm 0.195$ & 11 \\
\hline Thorium-230 (dissolved) & $1.02 \pm 0.242$ & $2.43 \pm 0.62$ & $1.76 \pm 0.336$ & $1.76 \pm 0.134$ & 11 \\
\hline Uranium (suspended) & $-.046 \pm 0.192$ & $.255 \pm 0.224$ & $.124 \pm 0.121$ & $.104 \pm 0.032$ & 10 \\
\hline Thorium-230 (suspended) & $-.026 \pm 0.106$ & $.136 \pm 0.122$ & $.066 \pm 0.065$ & $.057 \pm 0.018$ & 10 \\
\hline SR-90/Y-90 (dissolved) & $1.83 \pm 0.444$ & $2.98 \pm 0.409$ & $2.31 \pm 0.340$ & $2.38 \pm 0.111$ & 11 \\
\hline Cesium-137 (dissolved) & $2.49 \pm 0.52$ & $3.90 \pm 0.54$ & $3.17 \pm 0.52$ & $3.22 \pm 0.148$ & 11 \\
\hline SR-90/Y-90 (suspended) & $-.274 \pm 0.238$ & $.607 \pm 0.227$ & $.4155 \pm 0.177$ & $.341 \pm 0.079$ & 10 \\
\hline \multirow[t]{2}{*}{ Cesium-137 (suspended) } & $-.283 \pm 0.246$ & $.635 \pm 0.238$ & $.434 \pm 0.185$ & $.358 \pm 0.084$ & 10 \\
\hline & \multicolumn{5}{|c|}{ USGS 99} \\
\hline Uranium (dissolved) & $1.76 \pm 0.419$ & $5.08 \pm 1.22$ & $2.91 \pm 0.94$ & $3.08 \pm 0.270$ & 13 \\
\hline Thorium-230 (dissolved) & $1.27 \pm 0.280$ & $3.24 \pm 0.78$ & $2.15 \pm 0.398$ & $2.13 \pm 0.152$ & 13 \\
\hline Uranium (suspended) & $-.230 \pm 0.140$ & $.418 \pm 0.284$ & $.0005 \pm 0.105$ & $.031 \pm 0.063$ & 10 \\
\hline Thorium-230 (suspended) & $-.122 \pm 0.076$ & $.232 \pm 0.164$ & $0 \pm 0.056$ & $.019 \pm 0.036$ & 10 \\
\hline SR-90/Y-90 (dissolved) & $1.40 \pm 0.312$ & $2.64 \pm 0.400$ & $1.94 \pm 0.352$ & $2.03 \pm 0.098$ & 13 \\
\hline Cesium-137 (dissolved) & $1.88 \pm 0.412$ & $3.47 \pm 0.52$ & $2.55 \pm 0.462$ & $2.71 \pm 0.131$ & 13 \\
\hline SR-90/Y-90 (suspended) & $-.260 \pm 0.228$ & $.514 \pm 0.252$ & $.3405 \pm 0.176$ & $.260 \pm 0.082$ & 10 \\
\hline Cesium-137 (suspended) & $-.267 \pm 0.234$ & $.537 \pm 0.263$ & $.370 \pm 0.193$ & $.282 \pm 0.088$ & 10 \\
\hline
\end{tabular}


Table 11.-Statistical parameters for gross alpha-particle radioactivity, gross beta-particle radioactivity, radium-226, and radium-228, by well-Continued

\begin{tabular}{|c|c|c|c|c|c|}
\hline \multirow{2}{*}{$\begin{array}{l}\text { Radioactivity or } \\
\text { radionuclide }\end{array}$} & \multicolumn{5}{|c|}{ Statistical parameter } \\
\hline & Minimum & Maximum & Median & Mean & $\begin{array}{l}\text { Sample } \\
\text { size }\end{array}$ \\
\hline & \multicolumn{5}{|c|}{ USGS 102} \\
\hline Uranium (dissolved) & $1.98 \pm 0.444$ & $5.73 \pm 0.74$ & $3.755 \pm 0.424$ & $3.90 \pm 0.311$ & 12 \\
\hline Thorium-230 (dissolved) & $1.38 \pm 0.310$ & $4.11 \pm 0.54$ & $2.595 \pm 0.429$ & $2.73 \pm 0.228$ & 12 \\
\hline Uranium (suspended) & $-.092 \pm 0.180$ & $.215 \pm 0.192$ & $-.019 \pm 0.123$ & $.044 \pm 0.039$ & 9 \\
\hline Thorium-230 (suspended) & $-.052 \pm 0.102$ & $.117 \pm 0.106$ & $-.010 \pm 0.067$ & $.025 \pm 0.021$ & 9 \\
\hline SR-90/Y-90 (dissolved) & $2.18 \pm 0.372$ & $3.23 \pm 0.52$ & $2.62 \pm 0.310$ & $2.71 \pm 0.096$ & 12 \\
\hline Cesium-137 (dissolved) & $2.88 \pm 0.490$ & $4.28 \pm 0.58$ & $3.495 \pm 0.392$ & $3.61 \pm 0.131$ & 12 \\
\hline SR-90/Y-90 (suspended) & $-.07^{n+} n .220$ & $.586 \pm 0.250$ & $.095 \pm 0.237$ & $.168 \pm 0.072$ & 9 \\
\hline \multirow[t]{2}{*}{ Cesium-137 (suspended) } & $-.073 \pm 0.230$ & $.602 \pm 0.258$ & $.099 \pm 0.248$ & $.173 \pm 0.074$ & 9 \\
\hline & \multicolumn{5}{|c|}{ Water Supply INEL-1 } \\
\hline Uranium (dissolved) & $1.64 \pm 0.82$ & $3.40 \pm 1.21$ & $2.64 \pm 0.51$ & $2.65 \pm 0.141$ & 13 \\
\hline Thorium-230 (dissolved) & $1.26 \pm 0.63$ & $2.60 \pm 0.92$ & $1.82 \pm 0.353$ & $1.93 \pm 0.117$ & 13 \\
\hline Uranium (suspended) & $-.140 \pm 0.170$ & $.433 \pm 0.274$ & $.1785 \pm 0.175$ & $.134 \pm 0.076$ & 10 \\
\hline Thorium-230 (suspended) & $-.076 \pm 0.094$ & $.238 \pm 0.156$ & $.097 \pm 0.085$ & $.073 \pm 0.042$ & 10 \\
\hline SR-90/Y-90 (dissolved) & $2.54 \pm 0.448$ & $4.33 \pm 0.66$ & $3.63 \pm 0.58$ & $3.57 \pm 0.134$ & 13 \\
\hline Cesium-137 (dissolved) & $3.40 \pm 0.60$ & $5.80 \pm 0.88$ & $4.82 \pm 0.77$ & $4.74 \pm 0.182$ & 13 \\
\hline SR-90/Y-90 (suspended) & $.054 \pm 0.240$ & $.872 \pm 0.270$ & $.4085 \pm 0.180$ & $.486 \pm 0.090$ & 10 \\
\hline Cesium-137 (suspended) & $.055 \pm 0.247$ & $.911 \pm 0.283$ & $.422 \pm 0.187$ & $.505 \pm 0.094$ & 10 \\
\hline
\end{tabular}




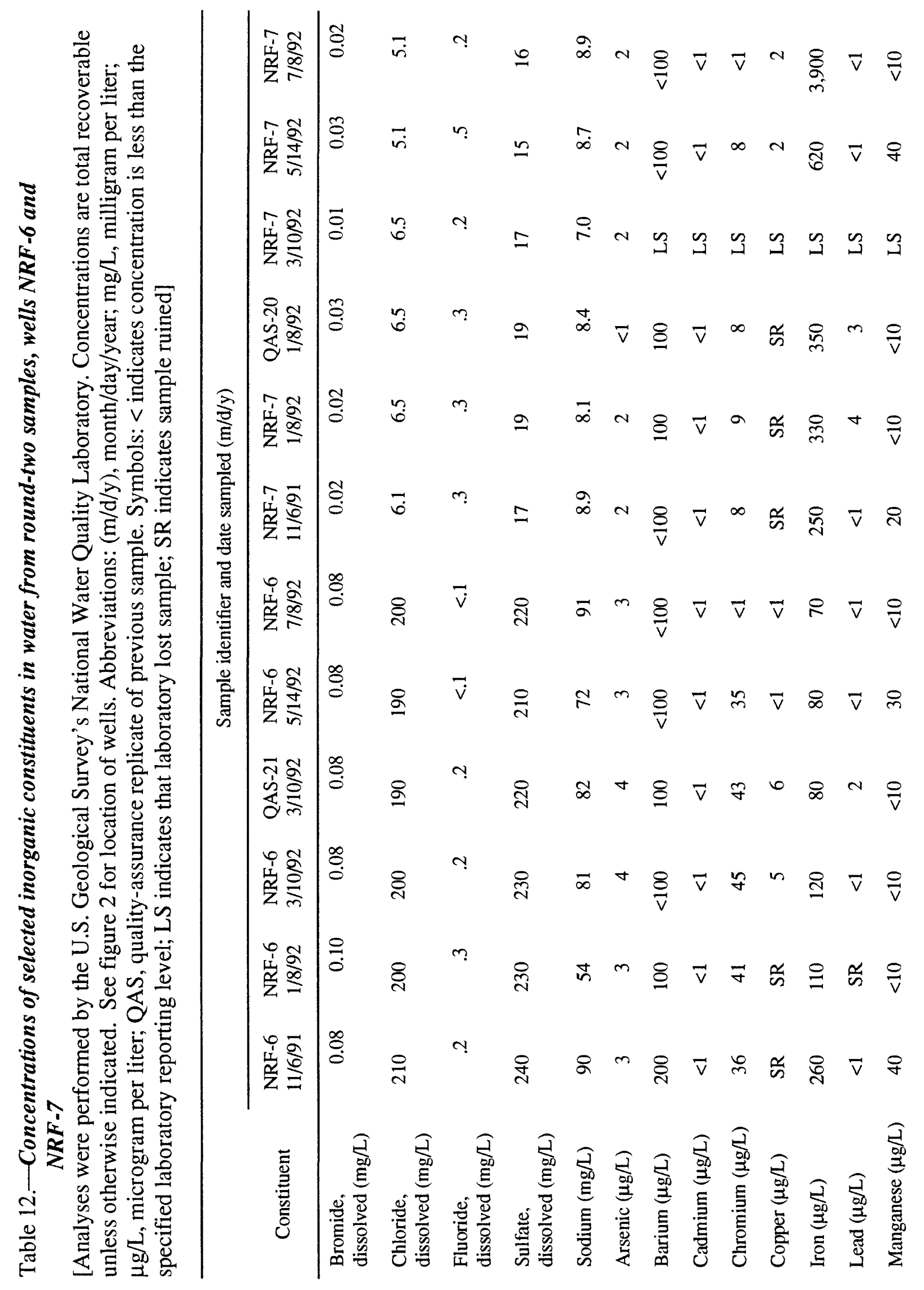




\begin{tabular}{|c|c|c|c|c|c|c|c|c|c|}
\hline & $\begin{array}{l}\frac{1}{2} \\
\frac{2}{2} \\
\frac{2}{2}\end{array}$ & $\vec{v} m$ & - & $\vec{v}$ & $\stackrel{\ominus}{v}$ & $\bar{\Xi}$ & $\vec{i}$ & ษִ & $\vec{i}$ \\
\hline & 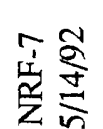 & $\vec{v}$ in & - & $\vec{v}$ & $\cong$ & $\overrightarrow{\stackrel{\vec{v}}{v}}$ & $\vec{i}$ & f. & $\bar{i}$ \\
\hline & 产突 & $\vec{v} \quad 3$ & - & 3 & 3 & $\because$ & $\overline{\vec{v}}$ & f. & $\overrightarrow{\mathrm{i}}$ \\
\hline & 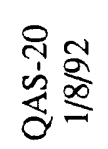 & $\vec{v} m$ & - & $\vec{v}$ & q & $\bar{i}$ & $\bar{i}$ & $\stackrel{\infty}{\eta}$ & $\bar{\sigma}$ \\
\hline ह & 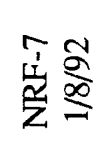 & $\vec{v}+$ & $\vec{v}$ & $\vec{v}$ & లి & $\vec{i}$ & $\overline{\vec{v}}$ & ले & $\delta$ \\
\hline 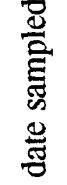 & 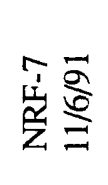 & $\vec{v} n$ & $\curvearrowright$ & $\vec{v}$ & $\stackrel{\ominus}{v}$ & $\tilde{\sigma}$ & $\vec{i}$ & के & $\overrightarrow{0}$ \\
\hline 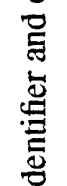 & 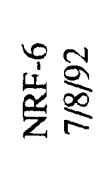 & $\vec{v} r$ & - & $\vec{v}$ & $\stackrel{\ominus}{v}$ & $\bar{a}$ & $\overline{\vec{v}}$ & $\stackrel{\infty}{-}$ & 5 \\
\hline 离 & 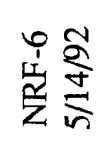 & $\vec{v} n$ & $\sim$ & $\bar{v}$ & $\cong$ & $\vec{i}$ & $\vec{i}$ & 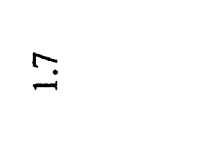 & $\stackrel{0}{0}$ \\
\hline & 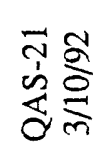 & $\vec{v}=$ & $\sim$ & $\overrightarrow{\mathrm{v}}$ & $\cong$ & $\tilde{\overbrace{}}$ & $\overline{\vec{v}}$ & $\Xi$ & 5 \\
\hline & 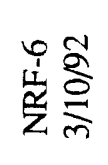 & $\vec{v} \vec{\sim}$ & $\sim$ & $\bar{v}$ & \& & $\tilde{\sigma}$ & $\bar{i}$ & $=$ & ¿. 1 \\
\hline & 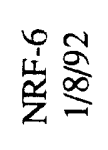 & $\vec{v} \varrho$ & $\sim$ & $\vec{v}$ & i & $\overline{\dot{v}}$ & $\overline{\vec{v}}_{\mathrm{v}}$ & $\stackrel{\varphi}{\longrightarrow}$ & 5 \\
\hline & 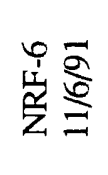 & $\vec{v} \vec{v}$ & $m$ & $\bar{v}$ & $\stackrel{\ominus}{v}$ & $\tilde{\sigma}$ & $\vec{i}$ & $\stackrel{\circ}{\longrightarrow}$ & 8 \\
\hline & 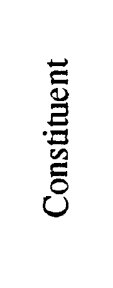 & 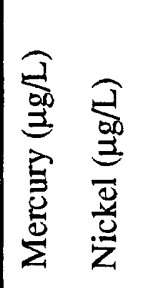 & 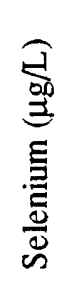 & 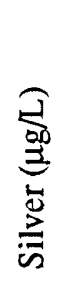 & 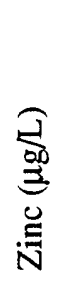 & 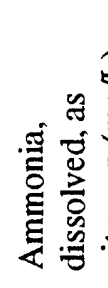 & 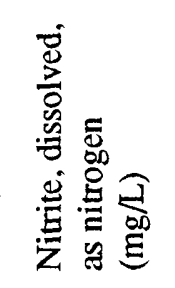 & 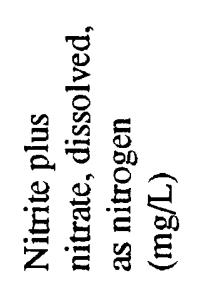 & 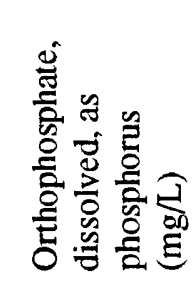 \\
\hline
\end{tabular}




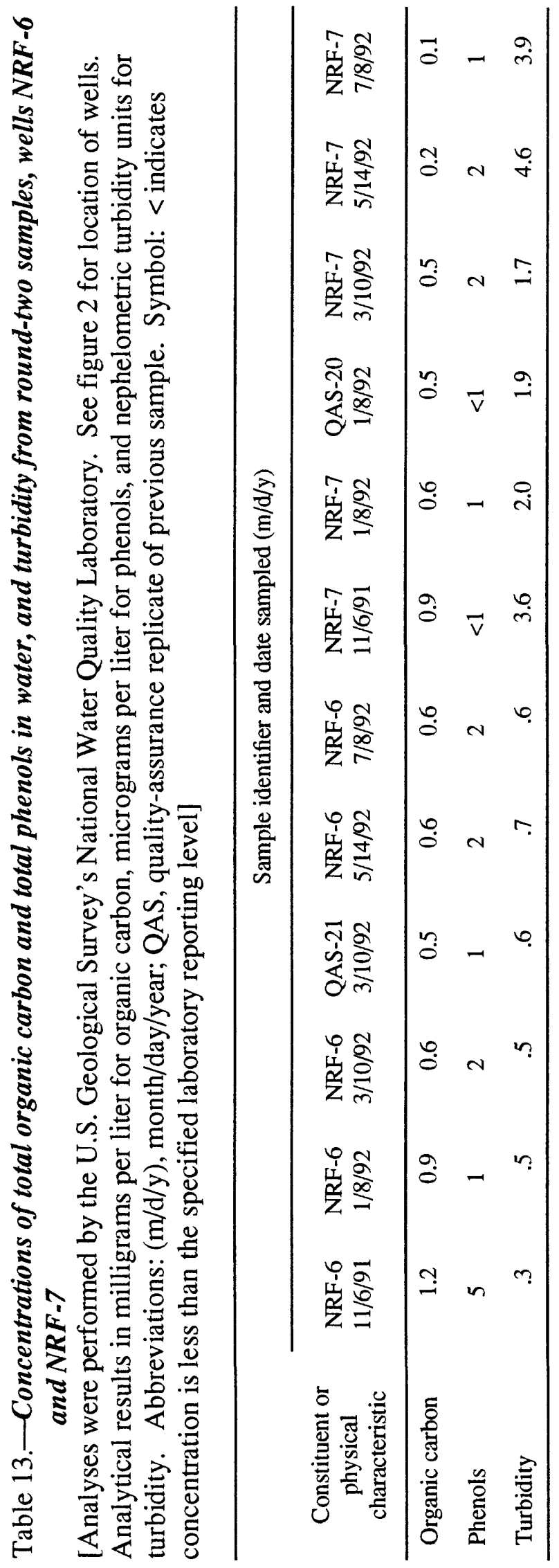


Table 14. Extractable acid and base/neutral organic compounds for which round-one and round-two water samples were analyzed

[Analyses were performed by the U.S. Geological Survey's National Water Quality Laboratory using gas chromatography to separate the compounds and mass spectrometry and flame ionization for identification and quantification. Initial extraction was with methylene chloride. Laboratory reporting levels are in micrograms per liter (C.A. Watterson and A.T. Kasuba, U.S. Geological Survey, written commun., 1993); 1,2,5,6-Dibenzanthracene was reported as Dibenzo $(a, h)$ anthracene for previous round-one and round-two samples]

\begin{tabular}{|c|c|c|c|}
\hline Compound & Reporting level & Compound & Reporting level \\
\hline Acenaphthene & 5 & 2,4-Dinitrophenol & 20 \\
\hline Acenaphthylene & 5 & 2,4-Dinitrotoluene & 5 \\
\hline Anthracene & 5 & 2,6-Dinitrotoluene & 5 \\
\hline Benzidine & 40 & Di-n-octyl phthalate & 10 \\
\hline Benzo (a) anthracene & 10 & 1,2-Diphenylhydrazine & 5 \\
\hline Benzo (b) fluoranthene & 10 & bis (2-Ethylhexyl) phthalate & 5 \\
\hline Benzo (k) fluoranthene & 10 & Fluoranthene & 5 \\
\hline Benzo (g,h,i) perylene & 10 & Fluorene & 5 \\
\hline Benzo (a) pyrene & 10 & Hexachlorobenzene & 5 \\
\hline 4-Bromophenyl phenyl ether & 5 & Hexachlorobutadiene & 5 \\
\hline Butyl benzyl phthalate & 5 & Hexachlorocyclopentadiene & 5 \\
\hline bis (2-Chloroethoxy) methane & 5 & Hexachloroethane & 5 \\
\hline bis (2-Chloroethyl) ether & 5 & Indeno $(1,2,3-c d)$ pyrene & 10 \\
\hline bis (2-Chloroisopropyl) ether & 5 & Isophorone & 5 \\
\hline 4-Chloro-3-methylphenol & 30 & 2-Methyl-4,6-dinitrophenol & 30 \\
\hline 2-Chloronaphthalene & 5 & Naphthalene & 5 \\
\hline 2-Chlorophenol & 5 & Nitrobenzene & 5 \\
\hline 4-Chlorophenyl phenyl ether & 5 & 2-Nitrophenol & 5 \\
\hline Chrysene & 10 & 4-Nitrophenol & 30 \\
\hline 1,2,5,6-Dibenzanthracene & 10 & n-Nitrosodimethylamine & 5 \\
\hline 1,2-Dichlorobenzene & 5 & n-Nitrosodi-n-propylamine & 5 \\
\hline 1,3-Dichlorobenzene & 5 & n-Nitrosodiphenylamine & 5 \\
\hline 1,4-Dichlorobenzene & 5 & Pentachlorophenol & 30 \\
\hline 3,3'-Dichlorobenzidine & 20 & Phenanthrene & 5 \\
\hline 2,4-Dichlorophenol & 5 & Phenol & 5 \\
\hline Diethyl phthalate & 5 & Pyrene & 5 \\
\hline Dimethyl phthalate & 5 & 1,2,4-Trichlorobenzene & 5 \\
\hline 2,4-Dimethylphenol & 5 & 2,4,6-Trichlorophenol & 20 \\
\hline Di-n-butyl phthalate & 5 & & \\
\hline
\end{tabular}


Table 15.-Concentrations of extractable acid and base/neutral organic compounds in water from round-one and round-two samples, wells $N R F-6$ and $N R F-7$

[Analyses were performed by the U.S. Geological Survey's National Water Quality Laboratory. Analytical results in micrograms per liter. Compounds listed are tentatively identified organic compounds (TIOC's); the reported concentration generally is accurate to one order of magnitude. Data for TIOC's in this report are based on a comparison of sample spectra with library spectra followed by visual examination by gas chromatograph/mass spectrometer analysts. TIOC data have not been confirmed by direct comparison with reference standards. Therefore, TIOC identification is tentative, and reported concentrations are semiquantitative. Sample identifier: see figure 2 for location of wells; QAS indicates quality-assurance replicate of previous sample. Date sampled: $\mathrm{m} / \mathrm{d} / \mathrm{y}$ indicates month/day/year. Retention time: time required for a compound to pass through the column of a gas chromatograph. Remarks: BRL indicates concentrations of all compounds in table 14 are less than the reporting level; CAS No. indicates Chemical Abstract Services number - no entry indicates CAS No. not listed in Pritt and Jones (1989) or on laboratory analytical result sheet]

\begin{tabular}{|c|c|c|c|c|c|}
\hline $\begin{array}{l}\text { Sample } \\
\text { identifier }\end{array}$ & $\begin{array}{l}\text { Date sampled } \\
\qquad(\mathrm{m} / \mathrm{d} / \mathrm{y})\end{array}$ & Compound & Concentration & $\begin{array}{c}\text { Retention } \\
\text { time (minutes) }\end{array}$ & Remarks \\
\hline \multirow[t]{4}{*}{ NRF-6 } & 9/9/91 & See remarks & & & BRL \\
\hline & $11 / 6 / 91$ & See remarks & & & BRL \\
\hline & $1 / 8 / 92$ & See remarks & & & BRL \\
\hline & $3 / 10 / 92$ & See remarks & & & BRL \\
\hline QAS-21 & $3 / 10 / 92$ & See remarks & & & BRL \\
\hline \multirow[t]{2}{*}{ NRF-6 } & $5 / 14 / 92$ & See remarks & & & BRL \\
\hline & $7 / 8 / 92$ & See remarks & & & BRL \\
\hline \multirow[t]{14}{*}{ NRF-7 } & $9 / 10 / 91$ & See remarks & & & BRL \\
\hline & $11 / 6 / 91$ & See remarks & & & BRL \\
\hline & $1 / 8 / 92$ & Hydrocarbon & 6 & 37.02 & \\
\hline & & Pentacosane & 100 & 41.35 & CAS. No. 629992 \\
\hline & & Hydrocarbon & 3 & 42.09 & \\
\hline & & Hexacosane & 200 & 42.67 & CAS No. 630013 \\
\hline & & Hydrocarbon & 6 & 43.37 & \\
\hline & & Hydrocarbon & 2 & 43.51 & \\
\hline & & Heptacosane & 300 & 43.96 & CAS No. 593497 \\
\hline & & Hydrocarbon & 10 & 44.61 & \\
\hline & & Hydrocarbon & 6 & 44.74 & \\
\hline & & Octacosane & 300 & 45.17 & CAS No. 630024 \\
\hline & & Nonacosane & 10 & 45.80 & CAS No. 630035 \\
\hline & & Hydrocarbon & 9 & 45.93 & \\
\hline
\end{tabular}


Table 15.-Concentrations of extractable acid and base/neutral organic compounds in water from round-one and round-two samples, wells NRF-6 and NRF-7-Continued

\begin{tabular}{|c|c|c|c|c|c|}
\hline $\begin{array}{l}\text { Sample } \\
\text { identifier }\end{array}$ & $\begin{array}{l}\text { Date sampled } \\
(\mathrm{m} / \mathrm{d} / \mathrm{y})\end{array}$ & Compound & Concentration & $\begin{array}{c}\text { Retention } \\
\text { time (minutes) }\end{array}$ & Remarks \\
\hline \multirow[t]{23}{*}{ NRF-7 } & $1 / 8 / 92$ (cont.) & Hydrocarbon & 300 & 46.34 & \\
\hline & & Triacontane & 10 & 46.95 & CAS No. 638686 \\
\hline & & Hydrocarbon & 6 & 47.09 & \\
\hline & & Triacontane & 200 & 47.52 & CAS No. 638686 \\
\hline & & Hydrocarbon & 10 & 48.23 & \\
\hline & & Alkane & 10 & 48.23 & \\
\hline & & Hydrocarbon & 8 & 48.39 & \\
\hline & & Hydrocarbon & 200 & 48.87 & \\
\hline & & Hydrocarbon & 9 & 49.42 & \\
\hline & & Hydrocarbon & 10 & 49.70 & \\
\hline & & Hydrocarbon & 7 & 49.89 & \\
\hline & & Hydrocarbon & 100 & 50.40 & \\
\hline & & $\begin{array}{l}\text { Nitrogen-containing } \\
\text { compound }\end{array}$ & 7 & 50.69 & \\
\hline & & Triacontane & 5 & 51.39 & CAS No. 638686 \\
\hline & & Hentriacontane & 4 & 51.63 & CAS No. 630046 \\
\hline & & Hydrocarbon & 70 & 52.19 & \\
\hline & & $\begin{array}{l}\text { Nitrogen-containing } \\
\text { compound }\end{array}$ & 4 & 52.59 & \\
\hline & & Hydrocarbon & 3 & 53.65 & \\
\hline & & Hydrocarbon & 30 & 54.28 & \\
\hline & & $\begin{array}{l}\text { Nitrogen-containing } \\
\text { compound }\end{array}$ & 3 & 54.83 & \\
\hline & & Hydrocarbon & 20 & 56.78 & \\
\hline & & Hydrocarbon & 3 & 59.77 & \\
\hline & & See remarks & & & BRL \\
\hline QAS-20 & $1 / 8 / 92$ & See remarks & & & BRL \\
\hline \multirow[t]{3}{*}{ NRF-7 } & $3 / 10 / 92$ & See remarks & & & BRL \\
\hline & $5 / 14 / 92$ & See remarks & & & BRL \\
\hline & 7/8/92 & See remarks & & & BRL \\
\hline
\end{tabular}


Table 16. Pesticides for which round-two water samples were analyzed

[Analyses were performed by the U.S. Geological Survey's National Water Quality Laboratory using the following methods: chlorophenoxy-acid herbicides by converting the compounds to methyl esters followed by identification with gas chromatography and an electron-capture detector; organochlorine insecticides and gross polychlorinated compounds by extracting with hexane and identification with gas chromatography using electron-capture detectors (Wershaw and others, 1987, p. 27-28 and 40). Laboratory reporting levels are from Pritt and Jones (1989). Abbreviation: $\mu \mathrm{g} / \mathrm{L}$, microgram per liter]

\begin{tabular}{|c|c|}
\hline \multicolumn{2}{|c|}{$\frac{\text { Organochlorine insecticides: reporting level is } 0.01 \mathrm{\mu g} / \mathrm{L} \text { except for chlordane and }}{\text { perthane }(0.1 \mathrm{\mu g} / \mathrm{L}) \text { and toxaphene }(1.0 \mathrm{\mu g} / \mathrm{L})}$} \\
\hline Aldrin & Heptachlor \\
\hline Chlordane & Heptachlor epoxide \\
\hline DDD & Lindane \\
\hline DDE & Methoxychlor \\
\hline DDT & Mirex \\
\hline Dieldrin & Perthane \\
\hline Endosulfan & Toxaphene \\
\hline Endrin & \\
\hline \multicolumn{2}{|c|}{ Gross polychlorinated compounds: reporting level is $0.1 \mathrm{ug} / \mathrm{L}$} \\
\hline \multicolumn{2}{|c|}{ Gross polychlorinated biphenyls ( $\mathrm{PCB}$ ) } \\
\hline \multicolumn{2}{|c|}{ Gross polychlorinated naphthalenes ( $\mathrm{PCN})$} \\
\hline \multicolumn{2}{|c|}{ Chlorophenoxy-acid herbicides: reporting level is $0.01 \mu \mathrm{g} / \mathrm{L}$} \\
\hline 2,4-D & Silvex \\
\hline 2,4-DP & $2,4,5-\mathrm{T}$ \\
\hline
\end{tabular}


Table 17.-Concentrations of radioactivity, dissolved radium-226, and dissolved radium-228 in water from round-two samples, wells NRF-6 and NRF -7 .

[Analyses were performed by the U.S. Geological Survey's National Water Quality Laboratory using the following methods: gross alpha- and gross beta-particle radioactivity by residue procedure; radium- 226 by radon emanation; and radium- 228 by separation and beta counting. Raw field samples were processed in laboratory prior to analyses. Analytical results and uncertainties - for example, 4.17 $\mathbf{0 . 6 2 5}$ - are in indicated units. Analytical uncertainties are reported as 1s. Concentrations that meet or exceed the reporting level of 3 times the $1 \mathrm{~s}$ value are shown in boldface type. Abbreviations: (m/d/y), month/day/year; $\mu \mathrm{g} / \mathrm{L}$, microgram per liter; pCi/L, picocurie per liter; Sr-90/Y-90, strontium-90 in equilibrium with yttrium-90. Sample identifier: see figure 2 for location of wells; QAS indicates quality-assurance replicate of previous sample]

\begin{tabular}{|c|c|c|c|c|c|}
\hline \multirow{3}{*}{$\begin{array}{l}\text { Sample } \\
\text { identifier }\end{array}$} & \multirow{3}{*}{$\begin{array}{c}\text { Date } \\
\text { sampled } \\
(\mathrm{m} / \mathrm{d} / \mathrm{y})\end{array}$} & \multicolumn{4}{|c|}{ Gross alpha-particle radioactivity } \\
\hline & & \multicolumn{2}{|c|}{ Dissolved } & \multicolumn{2}{|c|}{ Suspended } \\
\hline & & $\begin{array}{l}\text { as uranium } \\
\qquad(\mu \mathrm{g} / \mathrm{L})\end{array}$ & $\begin{array}{l}\text { as thorium-230 } \\
(\mathrm{pCi} / \mathrm{L})\end{array}$ & $\begin{array}{l}\text { as uranium } \\
(\mu \mathrm{g} / \mathrm{L})\end{array}$ & $\begin{array}{l}\text { as thorium-230 } \\
(\mathrm{pCi} / \mathrm{L})\end{array}$ \\
\hline \multirow[t]{3}{*}{ NRF-6 } & $11 / 6 / 91$ & $4.17 \pm 0.62$ & $2.92 \pm 0.441$ & $0.111 \pm 0.186$ & $0.058 \pm 0.099$ \\
\hline & $1 / 8 / 92$ & $4.33 \pm 0.62$ & $2.98 \pm 0.420$ & $-.135 \pm 0.164$ & $-.076 \pm 0.092$ \\
\hline & $3 / 10 / 92$ & $4.35 \pm 2.00$ & $3.35 \pm 1.54$ & $-.009 \pm 0.086$ & $-.005 \pm 0.046$ \\
\hline QAS-21 & $3 / 10 / 92$ & $2.88 \pm 0.51$ & $1.97 \pm 0.352$ & $.216 \pm 0.194$ & $.116 \pm 0.106$ \\
\hline \multirow[t]{2}{*}{ NRF-6 } & $5 / 14 / 92$ & $4.11 \pm 0.62$ & $2.86 \pm 0.434$ & $-.018 \pm 0.121$ & $-.010 \pm 0.064$ \\
\hline & $7 / 8 / 92$ & $3.47 \pm 0.59$ & $2.16 \pm 0.386$ & $.121 \pm 0.167$ & $.069 \pm 0.096$ \\
\hline \multirow[t]{2}{*}{ NRF-7 } & $11 / 6 / 91$ & $2.59 \pm 0.482$ & $1.86 \pm 0.346$ & $.265 \pm 0.247$ & $.167 \pm 0.159$ \\
\hline & $1 / 8 / 92$ & $1.85 \pm 0.422$ & $1.28 \pm 0.294$ & $.017 \pm 0.150$ & $.012 \pm 0.105$ \\
\hline QAS-20 & $1 / 8 / 92$ & $1.98 \pm 0.422$ & $1.36 \pm 0.293$ & $.094 \pm 0.191$ & $.065 \pm 0.132$ \\
\hline \multirow[t]{3}{*}{ NRF-7 } & $3 / 10 / 92$ & $.833 \pm 0.268$ & $.553 \pm 0.178$ & $.270 \pm 0.184$ & $.147 \pm 0.104$ \\
\hline & $5 / 14 / 92$ & $.947 \pm 0.280$ & $.621 \pm 0.184$ & $.494 \pm 0.242$ & $.408 \pm 0.214$ \\
\hline & $7 / 8 / 92$ & $2.02 \pm 0.442$ & $1.42+0.311$ & $.077 \pm 0.222$ & $.057 \pm 0.163$ \\
\hline
\end{tabular}


Table 17.-Concentrations of radioactivity, dissolved radium-226, and dissolved radium-228 in water from round-two samples, wells NRF-6 and NRF-7-Continued

\begin{tabular}{|c|c|c|c|c|c|}
\hline \multirow{3}{*}{$\begin{array}{c}\text { Sample } \\
\text { identifier }\end{array}$} & \multirow{3}{*}{$\begin{array}{c}\text { Date } \\
\text { sampled } \\
(\mathrm{m} / \mathrm{d} / \mathrm{y})\end{array}$} & \multicolumn{4}{|c|}{ Gross beta-particle radioactivity } \\
\hline & & \multicolumn{2}{|c|}{ Dissolved } & \multicolumn{2}{|c|}{ Suspended } \\
\hline & & $\begin{array}{c}\text { as } \mathrm{Sr}-90 / \mathrm{Y}-90 \\
(\mathrm{pCi} / \mathrm{L})\end{array}$ & $\begin{array}{l}\text { as cesium-137 } \\
(\mathrm{pCi} / \mathrm{L})\end{array}$ & $\begin{array}{c}\text { as } \mathrm{Sr}-90 / \mathrm{Y}-90 \\
(\mathrm{pCi} / \mathrm{L})\end{array}$ & $\begin{array}{c}\text { as cesium-137 } \\
(\mathrm{pCi} / \mathrm{L})\end{array}$ \\
\hline \multirow[t]{3}{*}{ NRF-6 } & $11 / 6 / 91$ & $5.65 \pm 0.86$ & $7.61 \pm 1.16$ & $.235 \pm 0.233$ & $.249 \pm 0.247$ \\
\hline & $1 / 8 / 92$ & $5.50 \pm 0.86$ & $7.24 \pm 1.14$ & $.016 \pm 0.236$ & $.017 \pm 0.244$ \\
\hline & $3 / 10 / 92$ & $5.53 \pm 0.78$ & $6.87 \pm 0.98$ & $.520 \pm 0.238$ & $.537 \pm 0.246$ \\
\hline QAS-21 & $3 / 10 / 92$ & $4.78 \pm 0.76$ & $5.87 \pm 0.94$ & $.403 \pm 0.254$ & $.421 \pm 0.266$ \\
\hline \multirow[t]{2}{*}{ NRF-6 } & $5 / 14 / 92$ & $4.71 \pm 0.87$ & $6.20 \pm 1.15$ & $.308 \pm 0.252$ & $.327 \pm 0.268$ \\
\hline & $7 / 8 / 92$ & $4.74 \pm 0.80$ & $6.35 \pm 1.07$ & $.186 \pm 0.240$ & $.198 \pm 0.254$ \\
\hline \multirow[t]{2}{*}{ NRF-7 } & $11 / 6 / 91$ & $2.62 \pm 0.347$ & $3.45 \pm 0.52$ & $.634 \pm 0.266$ & $.655 \pm 0.275$ \\
\hline & $1 / 8 / 92$ & $2.55 \pm 0.455$ & $3.34 \pm 0.54$ & $.676 \pm 0.261$ & $.696 \pm 0.269$ \\
\hline QAS-20 & $1 / 8 / 92$ & $2.61 \pm 0.353$ & $3.48 \pm 0.54$ & $.573 \pm 0.248$ & $.608 \pm 0.262$ \\
\hline \multirow[t]{3}{*}{ NRF-7 } & $3 / 10 / 92$ & $3.03 \pm 0.403$ & $4.12 \pm 0.54$ & $.325 \pm 0.244$ & $.344 \pm 0.258$ \\
\hline & $5 / 14 / 92$ & $2.72 \pm 0.362$ & $3.55 \pm 0.56$ & $.784 \pm 0.284$ & $.813 \pm 0.294$ \\
\hline & $7 / 8 / 92$ & $2.27 \pm 0.335$ & $3.03 \pm 0.498$ & $.586 \pm 0.263$ & $.607 \pm 0.272$ \\
\hline
\end{tabular}


Table 17.-Concentrations of radioactivity, dissolved radium-226, and dissolved radium-228 in water from round-two samples, wells NRF-6 and NRF-7-Continued

\begin{tabular}{cccc}
\hline $\begin{array}{c}\text { Sample } \\
\text { identifier }\end{array}$ & $\begin{array}{c}\text { Date } \\
\text { sampled } \\
(\mathrm{m} / \mathrm{d} / \mathrm{y})\end{array}$ & $\begin{array}{c}\text { Radium-226 } \\
(\mathrm{pCi} / \mathrm{L})\end{array}$ & $\begin{array}{c}\text { Radium-228 } \\
(\mathrm{pCi} / \mathrm{L})\end{array}$ \\
\hline NRF-6 & $11 / 6 / 91$ & $.026 \pm 0.040$ & $.171 \pm 0.140$ \\
& $1 / 8 / 92$ & $\mathbf{. 0 5 2} \pm \mathbf{0 . 0 0 7}$ & $-.044 \pm 0.121$ \\
& $3 / 10 / 92$ & $.017 \pm 0.006$ & $.232 \pm 0.140$ \\
QAS-21 & $3 / 10 / 92$ & $\mathbf{. 0 2 9} \pm \mathbf{0 . 0 0 4}$ & $.233 \pm 0.152$ \\
NRF-6 & $5 / 14 / 92$ & $\mathbf{. 0 3 8} \pm \mathbf{0 . 0 0 6}$ & $.259 \pm 0.143$ \\
& $7 / 8 / 92$ & $\mathbf{. 0 1 3} \pm \mathbf{0 . 0 0 4}$ & $.197 \pm 0.134$ \\
NRF-7 & $11 / 6 / 91$ & $.045 \pm 0.044$ & $.328 \pm 0.152$ \\
& $1 / 8 / 92$ & $\mathbf{. 0 4 7} \pm \mathbf{0 . 0 0 6}$ & $.175 \pm 0.128$ \\
QAS-20 & $1 / 8 / 92$ & $\mathbf{. 0 4 2} \pm \mathbf{0 . 0 0 7}$ & $.126 \pm 0.134$ \\
NRF-7 & $3 / 10 / 92$ & $\mathbf{. 0 4 3} \pm \mathbf{0 . 0 0 6}$ & $.315 \pm 0.138$ \\
& $5 / 14 / 92$ & $\mathbf{. 0 3 3 \pm \mathbf { 0 . 0 0 6 }}$ & $.110 \pm 0.122$ \\
& $7 / 8 / 92$ & $\mathbf{. 0 4 0} \pm \mathbf{0 . 0 0 6}$ & $.189 \pm 0.120$ \\
\hline
\end{tabular}


Table 18.-Concentrations of selected inorganic constituents in water from round-one samples, wells NRF-6 and NRF-7

[Analyses were performed by the U.S. Geological Survey's National Water Quality Laboratory. Concentrations are total recoverable unless otherwise indicated. See figure 2 for location of wells. Abbreviations: mg/L, milligram per liter; $\mu \mathrm{g} / \mathrm{L}$, microgram per liter. Symbols: < indicates concentration is less than the specified laboratory reporting level; NAL indicates constituent not determined by laboratory]

\begin{tabular}{|c|c|c|c|}
\hline Constituent & NRF-6 (9/9/91) & NRF-7 $(9 / 10 / 91)$ & Remarks \\
\hline Calcium $(\mathrm{mg} / \mathrm{L})$ & 140 & 30 & \\
\hline Potassium (mg/L) & NAL & 2.8 & \\
\hline Magnesium (mg/L) & 34 & 9.0 & \\
\hline Sodium $(\mathrm{mg} / \mathrm{L})$ & 88 & 9.3 & \\
\hline Bromide (mg/L) & .09 & .02 & dissolved \\
\hline Chloride $(\mathrm{mg} / \mathrm{L})$ & 220 & 6.7 & dissolved \\
\hline Fluoride (mg/L) & .2 & .2 & dissolved \\
\hline Sulfate (mg/L) & 240 & 14 & dissolved \\
\hline Aluminum $(\mu \mathrm{g} / \mathrm{L})$ & 10 & 400 & \\
\hline Arsenic $(\mu \mathrm{g} / \mathrm{L})$ & 3 & 2 & \\
\hline Barium $(\mu \mathrm{g} / \mathrm{L})$ & 100 & $<100$ & \\
\hline Beryllium $(\mu \mathrm{g} / \mathrm{L})$ & $<10$ & $<10$ & \\
\hline Cadmium $(\mu \mathrm{g} / \mathrm{L})$ & $<1$ & $<1$ & \\
\hline Chromium $(\mu \mathrm{g} / \mathrm{L})$ & 29 & 10 & \\
\hline Cobalt $(\mu \mathrm{g} / \mathrm{L})$ & $<1$ & $<1$ & \\
\hline Copper $(\mu \mathrm{g} / \mathrm{L})$ & 1 & 4 & \\
\hline Iron $(\mu \mathrm{g} / \mathrm{L})$ & 120 & 670 & \\
\hline Lead $(\mu \mathrm{g} / \mathrm{L})$ & $<1$ & $<1$ & \\
\hline Manganese $(\mu \mathrm{g} / L)$ & 20 & 20 & \\
\hline Mercury $(\mu \mathrm{g} / L)$ & $<.1$ & $<.1$ & \\
\hline Nickel $(\mu \mathrm{g} / \mathrm{L})$ & 7 & 9 & \\
\hline Selenium $(\mu \mathrm{g} / \mathrm{L})$ & 2 & $<1$ & \\
\hline Silver $(\mu \mathrm{g} / \mathrm{L})$ & $<1$ & $<1$ & \\
\hline Thallium $(\mu \mathrm{g} / \mathrm{L})$ & $<1$ & $<1$ & dissolved \\
\hline $\operatorname{Zinc}(\mu \mathrm{g} / \mathrm{L})$ & 10 & $<10$ & \\
\hline Ammonia as nitrogen $(\mathrm{mg} / \mathrm{L})$ & $<.01$ & .01 & \\
\hline Ammonia plus organic nitrogen as nitrogen $(\mathrm{mg} / \mathrm{L})$ & .3 & $<.2$ & \\
\hline Nitrite as nitrogen $(\mathrm{mg} / \mathrm{L})$ & $<.01$ & $<.01$ & \\
\hline Nitrite plus nitrate as nitrogen $(\mathrm{mg} / \mathrm{L})$ & 1.4 & .38 & \\
\hline Cyanide $(\mathrm{mg} / \mathrm{L})$ & $<.01$ & $<.01$ & dissolved \\
\hline Phosphorus as phosphorus (mg/L) & .06 & .154 & \\
\hline Phosphorus, orthophosphate (mg/L) & .02 & $<.01$ & \\
\hline Phosphorus, orthophosphate (mg/L) & .02 & NAL & dissolved \\
\hline
\end{tabular}


Table 19.-Concentrations of total recoverable anionic surfactants, total organic carbon, and total phenols in water, and turbidity of water, from round-one samples, wells $N R F-6$ and $N R F-7$

[Analyses were performed by the U.S. Geological Survey's National Water Quality Laboratory. See figure 2 for location of wells. Abbreviations: $\mathrm{mg} / \mathrm{L}$, milligram per liter; $\mu \mathrm{g} / \mathrm{L}$, microgram per liter]

\begin{tabular}{lcc}
\hline \multicolumn{1}{c}{ Constituent or physical characteristic } & NRF-6 (9/9/91) & NRF-7 (9/10/91) \\
\hline Anionic surfactants as methylene blue active substances $(\mathrm{mg} / \mathrm{L})$ & 0.06 & 0.01 \\
Organic carbon $(\mathrm{mg} / \mathrm{L})$ & .9 & .4 \\
Phenols $(\mu \mathrm{g} / \mathrm{L})$ & 2 & 1 \\
Turbidity as nephelometric turbidity units & .5 & 7.3 \\
\hline
\end{tabular}


Table 20.-Purgeable organic compounds for which round-one water samples were analyzed

[Analyses were performed by the U.S. Geological Survey's National Water Quality Laboratory using an analytical method that conforms to U.S. Environmental Protection Agency method 524.2. The laboratory reporting level for all compounds is 0.2 microgram per liter $(\mu \mathrm{g} / \mathrm{L})$ except methylene chloride which is $0.3 \mu \mathrm{g} / \mathrm{L}$ (Pritt and Jones, 1989). Concentrations of all listed compounds were less than the laboratory reporting levels except as follows: NRF-6 (September $9,1991)$ —chloroform $(0.4 \mu \mathrm{g} / \mathrm{L})$, tetrachloroethylene $(0.5 \mu \mathrm{g} / \mathrm{L})$, and toluene $(0.6 \mu \mathrm{g} / \mathrm{L})$; and NRF-7 (September 10, 1991) - 1,1,1-trichloroethane $(0.4 \mu \mathrm{g} / \mathrm{L})]$

\begin{tabular}{ll}
\hline \multicolumn{1}{c}{ Compound } & \multicolumn{1}{c}{ Compound } \\
\hline Benzene & Cis-1,3-Dichloropropene \\
Bromoform & Trans-1,3-Dichloropropene \\
Carbon tetrachloride & 1,3 -Dichloropropene \\
Chlorobenzene & Ethylbenzene \\
Chloroethane & Methyl bromide \\
2-Chloroethyl vinyl ether & Styrene \\
Chloroform & Methylene chloride \\
Chloromethane & $1,1,2,2$-Tetrachloroethane \\
Dibromochloromethane & Tetrachloroethylene \\
Dichlorobromomethane & Toluene \\
1,2-Dichlorobenzene & Trichlorofluoromethane \\
1,3-Dichlorobenzene & $1,1,1-$ Trichloroethane \\
1,4-Dichlorobenzene & $1,1,2-$ Trichloroethane \\
Dichlorodifluoromethane & Trichloroethylene \\
1,2-Dibromoethane & Vinyl chloride \\
1,1-Dichloroethane & Xylenes, mixed \\
1,2-Dichloroethane & \\
1,1-Dichloroethylene & \\
1,2-trans-Dichloroethylene & \\
1,2-Dichloropropane & \\
\hline & \\
\hline
\end{tabular}


Table 21.-Herbicides for which round-one water samples were analyzed

[Analyses were performed by the U.S. Geological Survey's National Water Quality Laboratory using the following methods: triazine herbicides by gas chromatography with a nitrogen phosphorus detector; chlorophenoxy-acid herbicides by converting the compounds to methyl esters followed by identification with gas chromatography and an electron-capture detector (Wershaw and others, 1987, p. 40, 47). Laboratory reporting levels are from C.A. Watterson and A.T. Kashuba (U.S. Geological Survey, written commun., 1993). Abbreviation: $\mu \mathrm{g} / \mathrm{L}$, microgram per liter]

\begin{tabular}{|c|c|c|c|}
\hline \multicolumn{4}{|c|}{ Triazine herbicides } \\
\hline Herbicide & $\begin{array}{l}\text { Reporting } \\
\text { level }(\mu \mathrm{g} / \mathrm{L})\end{array}$ & Herbicide & $\begin{array}{l}\text { Reporting } \\
\text { level }(\mu \mathrm{g} / \mathrm{L})\end{array}$ \\
\hline Alachlor & 0.2 & Hexazinone & 0.2 \\
\hline Ametryn & .1 & Metolachlor & .2 \\
\hline Atrazine & .1 & Metribuzin & .1 \\
\hline Bromacil & .2 & Prometon & .2 \\
\hline Butachlor & .1 & Prometryn & .1 \\
\hline Butylate & .1 & Propachlor & .1 \\
\hline Carboxin & .2 & Propazine & .1 \\
\hline Cyanazine & .2 & Simazine & .1 \\
\hline Cycloate & .1 & Simetryn & .1 \\
\hline De-ethylatrazine & .2 & Terbacil & .2 \\
\hline De-isopropylatrazine & .2 & Trifluralin & .1 \\
\hline Diphenamid & .1 & Vernolate & .1 \\
\hline \multicolumn{4}{|c|}{ Chlorophenoxy-acid herbicides: reporting level is $0.01 \mu \mathrm{g} / \mathrm{L}$} \\
\hline Herbicide & & Herbicide & \\
\hline $2,4-\mathrm{D}$ & & Silvex & \\
\hline 2,4-DP & & $2,4,5-\mathrm{T}$ & \\
\hline
\end{tabular}


Table 22.-Insecticides, benzene hexachlorides, gross polychlorinated compounds, and aroclors for which round-one water samples were analyzed

[Analyses were performed by the U.S. Geological Survey's National Water Quality Laboratory using the following methods: carbamate insecticides are extracted with methylene chloride, concentrated, and analyzed by high-performance liquid chromatography using a dual-channel variable-wavelength ultraviolet detector; organophosphorus compounds are extracted with hexane and determined on a gas chromatograph with flame-photometric detectors; and organochlorine compounds are extracted with hexane and determined by gas chromatography using electron-capture detectors (Wershaw and others, 1987, p. 27-28 and 49). Laboratory reporting levels are from Pritt and Jones (1989) except those for carbamate insecticides which are from C.A. Watterson and A.T. Kasuba (U.S. Geological Survey, written commun., 1993). Abbreviation: $\mu \mathrm{g} / \mathrm{L}$, microgram per liter]

Carbamate insecticides: reporting level is $0.5 \mu \mathrm{g} / \mathrm{L}$

$\begin{array}{lll}\text { Aldicarb } & \text { Carbofuran } & \text { 1-Naphthol } \\ \text { Aldicarb sulfone } & \text { 3-Hydroxycarbofuran } & \text { Oxamyl } \\ \text { Aldicarb sulfoxide } & \text { Methiocarb } & \text { Propham } \\ \text { Carbaryl (Sevin) } & \text { Methomyl } & \text { Propoxur }\end{array}$

Organophosphorus insecticides: reporting level is $0.01 \mu \mathrm{g} / \mathrm{L}$

$\begin{array}{lll}\text { Chlorpyrifos; Dursban } & \text { Fonofos } & \text { Phorate } \\ \text { Diazinon } & \text { Malathion } & \text { Phosphorotrithioate, S,S,S-tributyl-(DEF) } \\ \text { Disulfoton (Di-syston) } & \text { Methyl parathion } & \text { Trithion } \\ \text { Ethion } & \text { Parathion } & \end{array}$

Organochlorine insecticides: reporting level is $0.01 \mu \mathrm{g} / \mathrm{L}$ except for chlordane and perthane $(0.1 \mu \mathrm{g} / \mathrm{L})$, and toxaphene $(1.0 \mu \mathrm{g} / \mathrm{L})$.

$\begin{array}{lll}\text { Aldrin } & \text { Dieldrin } & \text { Lindane } \\ \text { Chlordane } & \text { Endosulfan } & \text { Methoxychlor } \\ \text { DDD } & \text { Endrin } & \text { Mirex } \\ \text { DDE } & \text { Heptachlor } & \text { Perthane } \\ \text { DDT } & \text { Heptachlor epoxide } & \text { Toxaphene }\end{array}$


Table 22.-Insecticides, benzene hexachlorides, gross polychlorinated compounds, and aroclors for which round-one water samples were analyzed-Continued

Benzene hexachlorides: reporting level is $0.01 \mu \mathrm{g} / \mathrm{L}$

alpha-Benzene hexachloride (alpha-BHC)

beta-Benzene hexachloride (beta-BHC)

delta-Benzene hexachloride (delta-BHC)

Gross polychlorinated compounds: reporting level is $0.1 \mu \mathrm{g} / \mathrm{L}$

Gross polychlorinated biphenyls (PCB)

Gross polychlorinated naphthalenes (PCN)

Aroclors: reporting level is $0.1 \mu \mathrm{g} / \mathrm{L}$

Aroclor 1016

Aroclor 1242

Aroclor 1260

Aroclor 1221

Aroclor 1248

Aroclor 1232

Aroclor 1254 
Table 23. Concentrations of radioactivity and selected radionuclides in water from roundone samples, wells NRF-6 and NRF-7

[Analyses were performed by the U.S. Geological Survey's National Water Quality Laboratory using the following methods: gross alpha- and gross beta-particle radioactivity by residue procedure; radium- 226 by radon emanation; radium- 228 by separation and beta counting; and tritium by liquid scintillation. Raw field samples were processed in laboratory prior to analysis. Analytical results and uncertainties-for example, 7.03 \pm 2.74 are in indicated units. Analytical uncertainties are reported as $1 \mathrm{~s}$. Concentrations which meet or exceed the reporting level of three times the $1 \mathrm{~s}$ value are shown in boldface type. See figure 2 for location of wells.

Abbreviations: $\mu \mathrm{g} / \mathrm{L}$, microgram per liter; $\mathrm{pCi} / \mathrm{L}$, picocurie per liter; $\mathrm{Sr}-90 / \mathrm{Y}-90$, strontium- 90 in equilibrium with yttrium-90]

\begin{tabular}{|c|c|c|}
\hline Radioactivity or radionuclide & NRF-6 $(9 / 9 / 91)$ & NRF-7 (9/10/91) \\
\hline \multicolumn{3}{|l|}{ Gross alpha-particle radioactivity } \\
\hline dissolved as uranium $(\mu \mathrm{g} / \mathrm{L})$ & $7.03 \pm 2.74$ & $2.54 \pm 0.484$ \\
\hline dissolved as thorium-230 (pCi/L) & $5.22 \pm 2.04$ & $1.77 \pm 0.336$ \\
\hline suspended as uranium $(\mu \mathrm{g} / \mathrm{L})$ & $.127 \pm 0.244$ & $.189 \pm 0.226$ \\
\hline suspended as thorium-230 $(\mathrm{pCi} / \mathrm{L})$ & $.075 \pm 0.144$ & $.174 \pm 0.214$ \\
\hline \multicolumn{3}{|l|}{ Gross beta-particle radioactivity } \\
\hline dissolved as $\mathrm{Sr}-90 / \mathrm{Y}-90(\mathrm{pCi} / \mathrm{L})$ & $1.85 \pm 0.68$ & $2.80 \pm 0.364$ \\
\hline dissolved as cesium-137 (pCi/L) & $2.43 \pm 0.89$ & $3.75 \pm 0.56$ \\
\hline suspended as $\mathrm{Sr}-90 / \mathrm{Y}-90(\mathrm{pCi} / \mathrm{L})$ & $-.035 \pm 0.240$ & $.928 \pm 0.286$ \\
\hline suspended as cesium-137 (pCi/L) & $-.036 \pm 0.252$ & $.963 \pm 0.297$ \\
\hline Radium-226, dissolved (pCi/L) & $.035 \pm 0.006$ & $.038 \pm 0.007$ \\
\hline Radium-228, dissolved (pCi/L) & $.122 \pm 0.155$ & $.266 \pm 0.165$ \\
\hline Tritium, total (pCi/L) & $124.8 \pm 12.8$ & $19.2 \pm 12.8$ \\
\hline
\end{tabular}

